\title{
Nanobio Silver: Its Interactions with Peptides and Bacteria, and Its Uses in Medicine
}

Sonja Eckhardt,* Priscilla S. Brunetto, Jacinthe Gagnon, Magdalena Priebe, Bernd Giese, and Katharina M. Fromm*

Department of Chemistry, University of Fribourg, Chemin du Musée 9, 1700 Fribourg, Switzerland

\section{INTRODUCTION}

Silver, known in metallic form since antiquity, has very early been recognized by mankind for its antimicrobial properties, a phenomenon observed, for example, in the context of drinking water (a silver coin in a well), food (silver cutlery, water storage recipients), and medicine (silver skull plates, teeth). ${ }^{1}$ Silver compounds were also shown to be useful. For example, dilute solutions of silver nitrate served long, and still do in some countries, as antimicrobial ointment to be instilled into 
newborn babies' eyes to prevent contraction of gonorrhea from the mother. ${ }^{1}$

Used frequently until the middle of the 20th century, silver and its compounds were then replaced by the newly discovered antibiotics and nearly forgotten for almost 50 years. However, one rare example for the use of silver in this time period is silver sulfadiazine. Upon increasing bacterial resistance against current antibiotics, and with the steady development of nanoscale science, research on silver and its compounds has regained in interest, ${ }^{2}$ however, facing the challenge to catch up the time during which research activities in that area were low. Today, silver presents itself as bulk material, as nanoparticles, clusters, and a large variety of silver compounds that are available for numerous applications, one of them being in medicine.

For ca. 20 years, the number of publications dealing with silver in medical applications is increasing steadily; for example, the number of papers corresponding to a search on "silver in medicine" on the Web of Science database in April 2012 is given as below five in 1993, and above 60 in 2011, with ca. 1900 citations in 2011. These publications involve scientists from all areas: physicists, biochemists, chemists, materials scientists, microbiologists, and medical doctors, all tackling the problem from their respective points of view and with their own methods, respectively, highlighting applications from materials, analytics, detection, and diagnosis to treatment or drug delivery. This is, for example, reflected in a large number of different assays described to study the antimicrobial properties, the biocompatibility, and the toxicity in vitro and in vivo, based on many different bacterial strains and cell lines and leading to sometimes opposite results. If chemists, physicists, and material scientists tend to know the respective silver compounds used in their study very well, they usually limit their biological studies to in vitro antimicrobial tests. ${ }^{3-7}$ On the other hand, medically oriented reports are less clear about the exact materials used, and present more advanced in vitro as well as in vivo results, the latter of which are sometimes contradictory to in vitro data. ${ }^{8,9}$ Besides, the research at different scales, for example, at the macro- and microscopic level (phenomenological observations), on the one hand, and the molecular studies, on the other hand, is not always congruent.

Recent reviews and books covered so far individual aspects of, for example, silver in medicine, ${ }^{10-12}$ silver coordination compounds, ${ }^{13}$ and biomaterials containing silver. ${ }^{14}$ Other reports on nanotechnology and its use in medicine contain also chapters dedicated to silver. ${ }^{15-17}$

We thus felt it timely to attempt to bring together these different aspects, to collect the data out in the literature, and to link and present these results of the current state of the art together with the remaining challenges. This Review addresses thus many readers: the chemist will learn about the molecular effects of silver in biological environments; the materials scientist who is interested in making nanodevices may just as well find interesting examples herein as the microbiologist or medical doctor who wants to find out about the material composition and its side effects. We focus on four main aspects: (i) the interaction of silver at a molecular level, on the one hand, (ii) at a cellular level, on the other hand, (iii) literature dealing with nanomaterials from which we can learn and get clues on the functioning of silver at all levels, and (iv) studies on the biocompatibility of silver. These parts are followed by a critical discussion of the state of knowledge.
For silver and its compounds, the following sections will elucidate on the molecular interactions of biomolecules with silver ions and silver nanoparticles (AgNPs), focusing on peptides as main reaction partners in cells, then on bacterial interactions with silver, on the use of silver in biomaterials, and on the biocompatibility aspects of this metal and its compounds. We will thus shine light on the different aspects of silver in medicine, exploring the different levels of activity, from the molecular to the cellular and micrometer dimensions. Silver presents itself in different forms; among them ionic silver and AgNPs are the most frequently discussed in the literature. We will also present bulk silver surfaces as well as the so-called nanosilver, a recently described, apparently new class of silver. For the latter, we will discuss it wherever we find the, still rare, literature.

From a chemical point of view, it is convenient to first highlight interactions of silver at the molecular level because it is the basis for the interactions at higher levels. Thus, in the next section, we will start the journey with the examination of the silver binding to amino acids and peptides.

\section{PEPTIDES AND SILVER}

\subsection{Silver Binding to Amino Acids and Peptides}

The interaction of metal ions with biomolecular targets, for example, amino acids, peptides, or proteins, is known to play a fundamental role in many biological processes such as electron transfer reactions, oxygen transport, as well as metal transport and storage. Further on, the inhibition of enzymes by metal complexes with labile ligands is well-known. It functions by ligand exchange reactions, where the labile ligand present in the administered drug is replaced by the targeted enzyme, a principle used in many metal-based drugs. ${ }^{18}$ It is therefore one of the most important processes in bioinorganic chemistry. ${ }^{19}$ Although silver ions do not seem to be involved in natural systems, their medical use makes them an important target. $\mathrm{Ag}(\mathrm{I})$ can be classified as a soft cation according to Pearson, ${ }^{20}$ and therefore it prefers to bind to polarizable, so-called soft, ligands. In addition, it is able to form strong $\sigma-\pi$-bonds through back-donation of the electrons located in its d-orbitals to the $\pi^{*}$-orbitals of the ligand. In terms of electronic structure, $\mathrm{Ag}(\mathrm{I})$ is closely related to $\mathrm{Cu}(\mathrm{I})$ because both ions have the same outer electronic configuration with a closed-shell structure $\left(\mathrm{d}^{10}\right)$. Copper as trace element plays a fundamental role as cofactor in all organisms. ${ }^{21}$ Because the presence of larger amounts of free copper ions is toxic for the cells, many peptides and proteins are involved in its homeostasis. ${ }^{21}$ Because of the close chemical relation between $\operatorname{Ag}(\mathrm{I})$ and $\mathrm{Cu}(\mathrm{I})$, it is not surprising that silver has been also found to bind to peptides and proteins. Because amino acids are the small subunits used to construct peptides and proteins, it is reasonable to first have a closer look at their interaction with silver ions to answer the question on how $\mathrm{Ag}(\mathrm{I})$ binds to their polymeric form.

2.1.1. Silver Binding to Amino Acids - Theory. The silver complexes of all common 20 naturally occurring $\alpha$-amino acids have been calculated using, for example, the hybrid density functional theory (DFT) $)^{22,23}$ or the quantitative structure-property relationship methodology. ${ }^{24} \mathrm{~A}$ comparison of these data is not easy, due to the different levels of calculation, varying basis sets, or the nonidentical treatment of the basis set superposition error. Nevertheless, these theoretical studies reveal a common trend, with the three basic amino acids arginine, lysine, and histidine being the strongest silver ion 
binders, while the nonpolar aliphatic amino acids with the exception of methionine have the weakest silver ion affinity among the naturally occurring amino acids, whose side chains are shown in Figure 1. This affinity is defined as the binding

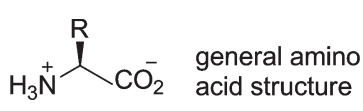
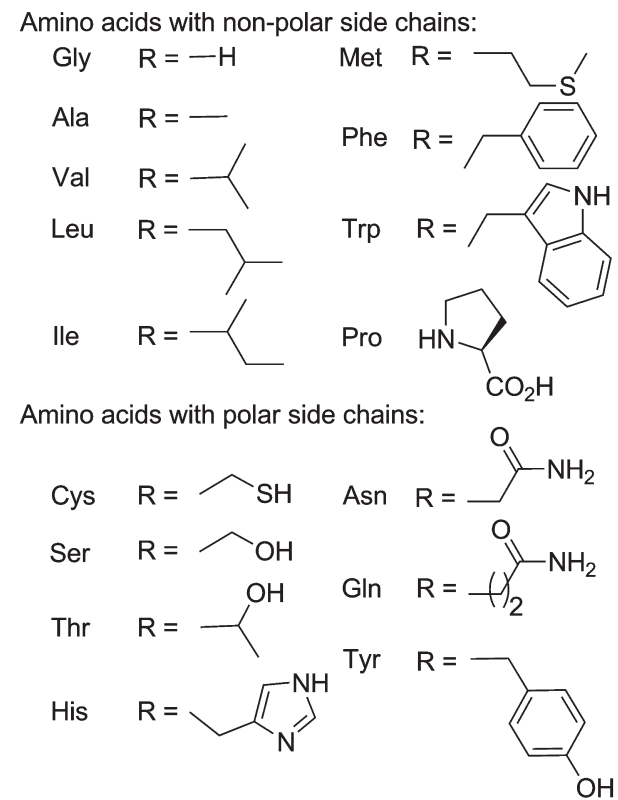

Amino acids with charged polar side chains:

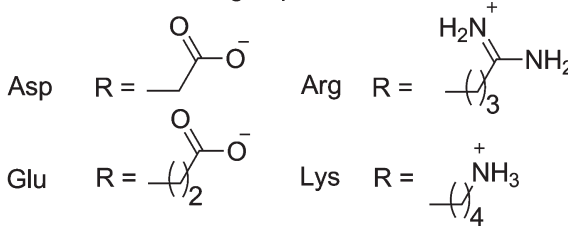

Figure 1. The 20 common natural $\alpha$-amino acids classified according to their side chain $\mathrm{R}$.

energy at $298 \mathrm{~K}$. The calculated silver ion affinity of all 20 natural amino acids on the basis of a 1:1 stoichiometry can be found in Table $1 .{ }^{23}$ Most interesting for a classical coordination chemist, cysteine is not among the calculated main candidates for silver binding even though the sulfur atom is considered as the softest group present in proteins. Cysteine is therefore, together with methionine, often considered as preferential binding site for silver ions in proteins, ${ }^{25}$ an assumption that is not confirmed by theory.

The total binding energy can be decomposed into two energy terms, one for the preorganization of the amino acid and the second for the interaction between amino acid and cation. Because this latter interaction is attractive, this term is always negative and may compensate for the preorganization energy, which is positive due to the higher energy of the amino acid in the complexed form (less degrees of freedom) as compared to their free state. The order in theoretical binding energies for the metal ion-amino acid complex formation is very similar for $\operatorname{Ag}(\mathrm{I})$ and $\mathrm{Cu}(\mathrm{I})$, the main exceptions being proline and serine, both having a higher affinity toward silver as compared to copper with respect to the other amino acids. Jover et al. ${ }^{23}$ also compared the affinity toward silver with the binding affinities of the amino acids for other monocharged cations, identifying the
Table 1. Calculated Enthalpies $\Delta H^{\circ}$ and Free Energies $\Delta G^{\circ}$ $\left(\mathrm{kJ} \mathrm{mol}^{-1}\right)$ for the Amino Acid-Ag(I) Complexes

\begin{tabular}{lll}
\multicolumn{1}{c}{$\alpha$-amino acid } & $\Delta H^{\circ a}$ & $\Delta G^{\circ a}$ \\
glycine (Gly) & 206.1 & 170.1 \\
alanine (Ala) & 212.8 & 176.8 \\
valine (Val) & 216.1 & 181.0 \\
leucine (Leu) & 219.5 & 185.6 \\
isoleucine (Ile) & 221.1 & 188.9 \\
serine (Ser) & 224.5 & 190.2 \\
cysteine (Cys) & 230.2 & 194.4 \\
threonine (Thr) & 233.2 & 199.8 \\
aspartic acid (Asp) & 232.4 & 199.0 \\
proline (Pro) & 234.5 & 199.8 \\
phenylalanine (Phe) & 236.2 & 198.6 \\
glutamic acid (Glu) & 239.9 & 203.1 \\
tyrosine (Tyr) & 239.9 & 202.3 \\
asparagine (Asn) & 250.8 & 217.4 \\
tryptophan (Trp) & 260.0 & 221.5 \\
methionine (Met) & 262.1 & 219.9 \\
glutamine (Gln) & 264.2 & 225.5 \\
histidine (His) & 284.2 & 249.1 \\
lysine (Lys) & 296.8 & 260.8 \\
arginine (Arg) & 336.5 & 279.8 \\
${ }^{a}$ Estimated from ref 23. & & \\
\hline
\end{tabular}

order $\mathrm{Cu}(\mathrm{I})>\mathrm{Li}(\mathrm{I})>\operatorname{Ag}(\mathrm{I})>\mathrm{Na}(\mathrm{I})>\mathrm{K}(\mathrm{I})$ for most of the amino acids. The calculated binding affinities also take into account possible coordination modes between $\operatorname{Ag}(\mathrm{I})$ and the amino acid. While some metal ions are able to adopt different modes, others tend to prefer a single coordination geometry. These preferences in terms of coordination are thought to be one important factor on how nature realizes the metal ion selectivity of metal ion-containing enzymes, which is indispensable for their biological function. The toxicity of some metals, which are usually not present in biological systems, is often based on their ability to occupy these (under biological conditions) metal-specific binding sites through effective competition with the biologically relevant metal ion and strong coordination to its binding site. ${ }^{26}$ In principle, there can be up to three different coordination sites present in an amino acid: (i) the amino nitrogen-donor at the $\mathrm{N}$-terminus, (ii) the oxygen atoms of the carboxylic group of the $\mathrm{C}$-terminus, and if present (iii) the heteroatom-containing side chains. A selection of possible coordination modes for the $\mathrm{Ag}(\mathrm{I})$ complexes of amino acids is given in Figure 2. ${ }^{24}$ The binding can either occur without or with the help of the side chain. In the first case, the amino acid acts as bidentate ligand either via both termini (1 and 2) or via both oxygen atoms of the carboxylic group of the C-terminus (3 and 4 ). If the silver binding occurs with the help of the side chain, the amino acid can either act as tridentate (5) or bidentate ligand (6 and 7).

In theory, monodentate coordination via only one heteroatom is possible as well, leading to a $2: 1$ complex between the amino acid and silver with respect to the preferred metal ion coordination number of two of the metal ion. However, because there is no stabilizing contribution due to chelation for the monodentate forms, they are expected to be higher in energy. Hopkinson divided the structures of silveramino acid complexes obtained by theoretical studies into three major categories. ${ }^{27}$ The first one represents five-membered cyclic structures with the silver ion dicoordinated by the two termini (1-3). Members of the second category coordinate 


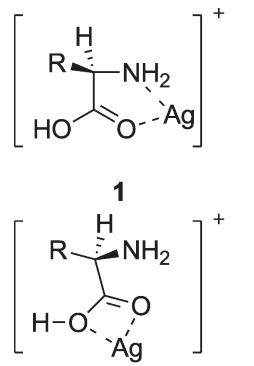

3
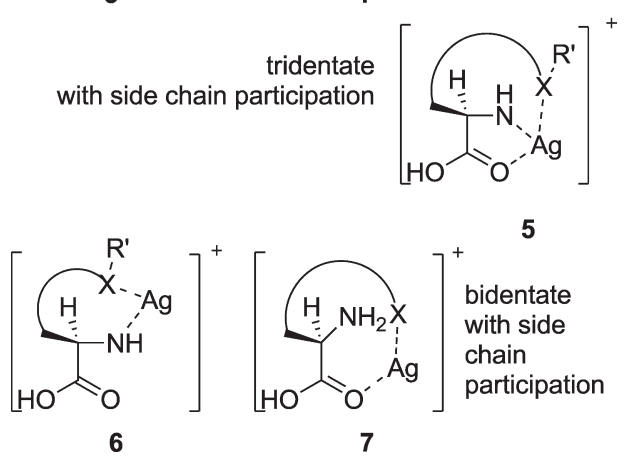

5

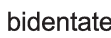

with side

chain participation
Figure 2. Selection of possible coordination modes between $\mathrm{Ag}(\mathrm{I})$ and amino acids. $\mathrm{R}$ represents the side chain of the amino acid in general, if it does not participate in the silver binding, while $\mathrm{X}$ represents the coordinating heteroatom present in the side chain.

$\mathrm{Ag}(\mathrm{I})$ by three or four atoms, yielding structures with multiple rings (5), while the members of the last group bind $\mathrm{Ag}(\mathrm{I})$ via the carboxylate of their zwitterionic form in a bidentate fashion (4). Further on, the members of the first two categories have been described as charge-solvated because the silver ion is well solvated by the amino acid in the gas phase, while the last group forms salt-bridge structures. ${ }^{22}$ Structure 1 was found to be the predominant coordination mode of unpolar aliphatic amino acids with hydrogen or alkyl groups in their side chain. The $\mathrm{Ag}(\mathrm{I})$ is symmetrically coordinated by the two termini with almost identical $\mathrm{Ag}-\mathrm{N}$ and $\mathrm{Ag}-\mathrm{O}$ distances, for example, 2.418 and $2.390 \AA$ in case of the $\mathrm{Ag}(\mathrm{I})$-glycine complex. ${ }^{26}$ The $\mathrm{Ag}(\mathrm{I})$ - proline complex is the only case where the salt bridge structure 4 is favored over the charged solvated forms. ${ }^{23}$ This is probably, on the one hand, due to the constraints of the fivemembered ring that do not allow the optimum orientation of the lone pair on the nitrogen atoms, and, on the other hand, due to the larger basicity of the secondary amine. All of the remaining amino acids are predicted to coordinate in a tridentate fashion with side chain participation (5). In these cases, the silver-coordination bonds with the termini are elongated as compared to the bidentate structures of the unpolar, aliphatic amino acids (by ca. $0.050 \AA$ ) due to the participation of the third coordination site. For the four amino acids possessing the highest proton affinities (Arg, Lys, His, and $\mathrm{Gln}$ ), the additional $\mathrm{Ag}-\mathrm{X}$ bond is calculated to be shorter than those with the termini, indicating a stronger binding of the side chain.

After having discussed the theoretical work on coordination of amino acids toward silver, we will in the next subsection focus on experimental efforts undertaken in this research area.

2.1.2. Silver Binding to Amino Acids - Experiment. To our current knowledge, the only experimental data for the $\mathrm{Ag}(\mathrm{I})$ affinity of all 20 naturally occurring amino acids (with the exception of Cys) have been provided by Siu and co-workers, ${ }^{28}$ who also determined the binding energies of the silver ion to simple alcohols and amides. ${ }^{27}$ They determined the relative silver ion affinities of the amino acids using relative $\Delta G^{\circ}$ values obtained by the kinetic method developed by Cooks and coworkers. ${ }^{29}$ The basis of this method is the competitive decomposition of a set of ion-bound heterodimers, whose general structure in case of the determination of silver binding affinities can be described as $\left[\mathrm{Aaa}_{x} \mathrm{AgAaa}_{y}\right]^{+}\left(\mathrm{Aaa}_{x}\right.$ and $\mathrm{Aaa}_{y}$ representing two different amino acids). The so-obtained experimental data ${ }^{28}$ are in good agreement with the previously described DFT calculations ${ }^{23}$ (Table 1).

Considering the number of amino acids, possible coordination modes (see section 2.1.1) and crystallization conditions, the structural information for silver-amino acid complexes based on X-ray structures is still rare. This is at first glance quite surprising because a detailed analysis of the coordination chemistry of amino acids is a prerequisite for the understanding of bioinorganic reaction mechanisms since they serve as model compounds for peptide-silver interactions. ${ }^{30}$ However, the relatively little amount of published crystal structures summarized in Table $2^{31-41}$ can be explained by the fact that the resulting compounds are difficult to synthesize because they are light sensitive in solution and poorly soluble in common solvents. ${ }^{39}$ Nomiya and co-workers recently showed that for alanine and asparagine the light stability of the silver complex can be enhanced with additional triphenylphosphine ligands ${ }^{38}$ or by acetylation of the N-terminus ${ }^{40}$ of the amino acid. To further classify the binding modes of $\mathrm{Ag}(\mathrm{I})$ in these structures (with the exception of sulfur-containing compounds), the following classification was proposed: ${ }^{39}$ the silver ion is involved (i) only in $\mathrm{Ag}-\mathrm{O}$ bonds (bond length $<2.6 \AA$ ), ${ }^{42}$ (ii) only in $\mathrm{Ag}-\mathrm{N}$ bonds (bond length $<2.5 \AA$ ) ${ }^{42}$ or (iii) in both $\mathrm{Ag}-\mathrm{O}$ and $\mathrm{Ag}-\mathrm{N}$ bonds.

The summary in Table 2 shows that the latter class is the most frequent one. Further on, it reveals that in the published structures the coordination geometry around the silver ion is, as expected, preferentially linear, leading either to $1 \mathrm{D}$ coordination polymers or to dimeric structures (e.g., for a silver to amino acid ratio of 1:2). For some of the presented structures, the $\mathrm{Ag}-\mathrm{Ag}$ distance is so short that the interaction of both $\mathrm{d}^{10}$ ions becomes discussable ${ }^{43,44}$ because the bonds are shorter than the van der Waals diameter of $3.44 \AA^{45}$ and in the range of the distance of $2.88 \AA^{46}$ found in metallic silver. It is surprising that among the few published structures there is a reasonable amount of structures with amino acids, which were calculated to be weak silver binders because they allow no side chain participation.

2.1.3. Silver Binding to Peptides. Going from amino acids to peptides, the number of coordination sites per molecule increases and the backbone amide can function as an additional coordination site as well. Shoeib and coworkers ${ }^{26,47}$ investigated the structure and free energy of diand tripeptides by means of DFT calculations. For the investigated silver-peptide complexes, they found structures at low energy minima with coordination numbers between two and four. Tricoordinated systems were found in the case of dipeptides, while tri- and tetracoordination were observed for tripeptides. As one might expect, experimental data based on Xray crystallography are even less abundant for silver-peptide complexes than for amino acids. To our current knowledge, the only published structure is the one published by Acland and Freeman $^{31}$ for Gly-Gly, the simplest of all dipeptides. At $\mathrm{pH}=$ 6, this dipeptide forms a dimeric complex 8 in which two silver 


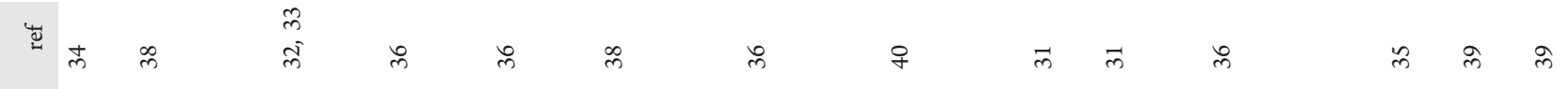

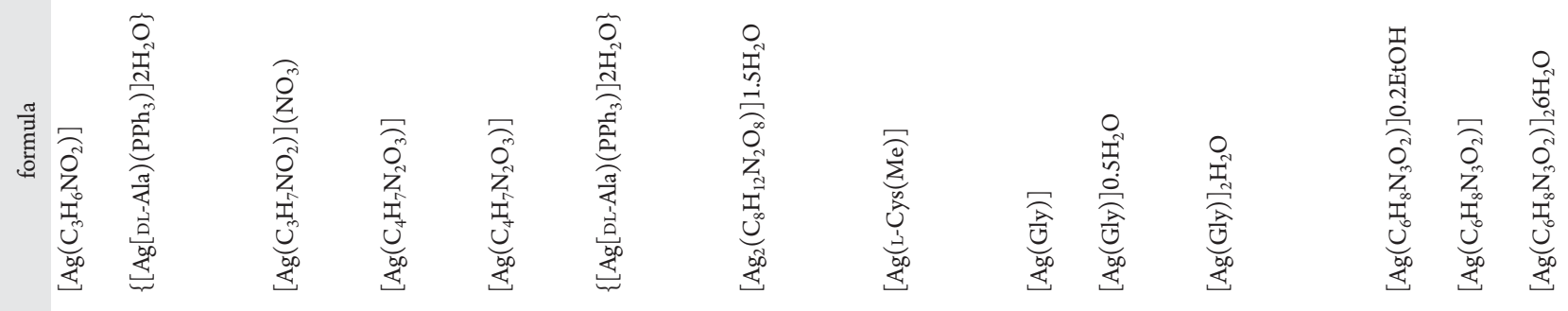

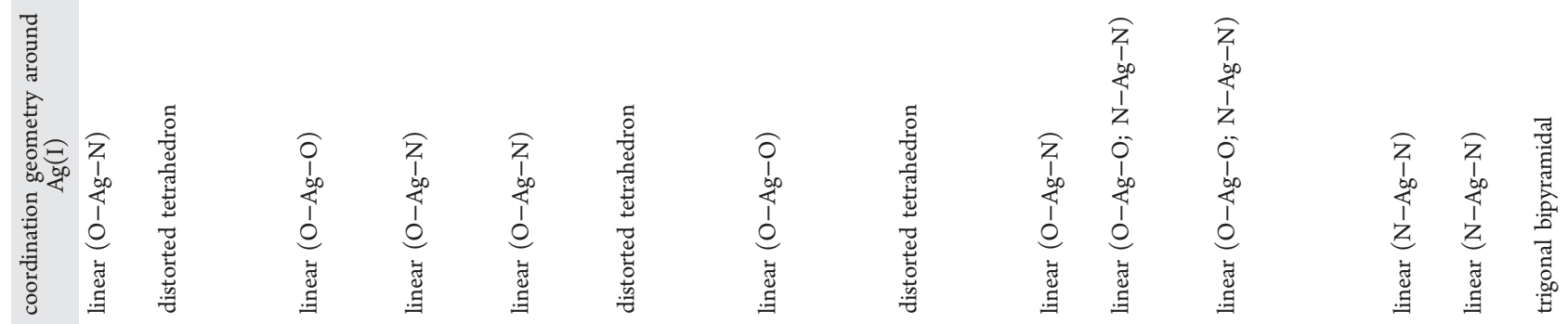

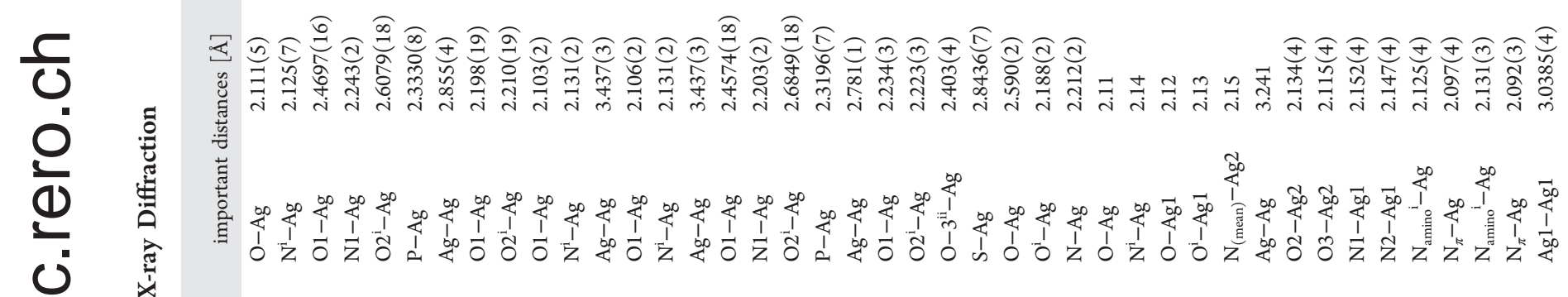

을

要
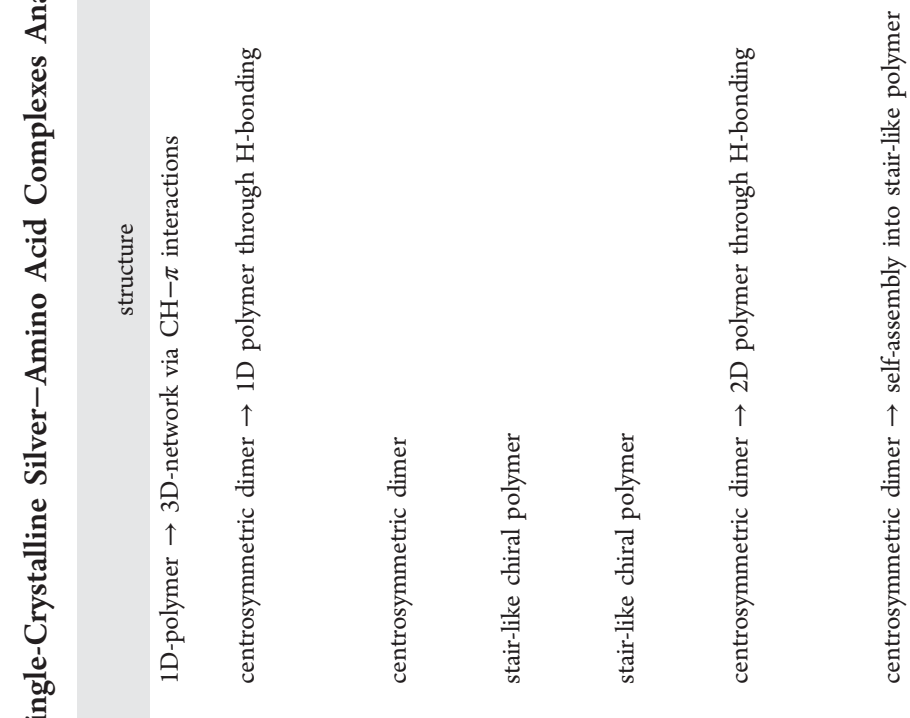

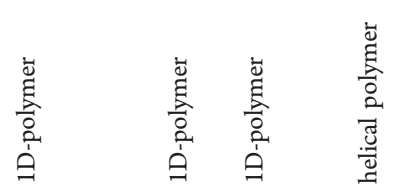

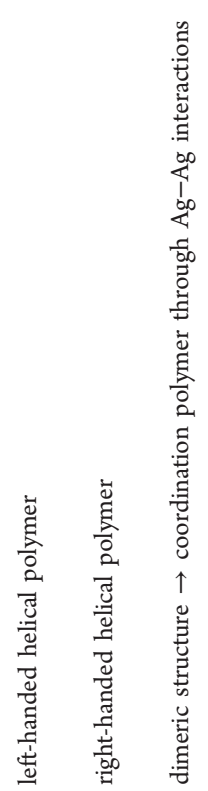

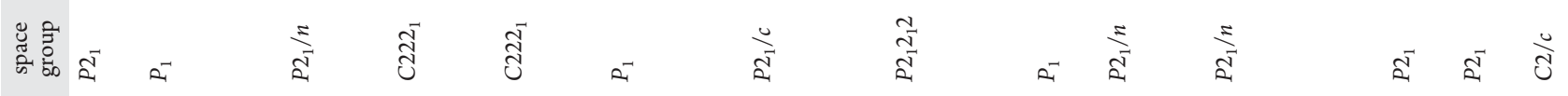

总

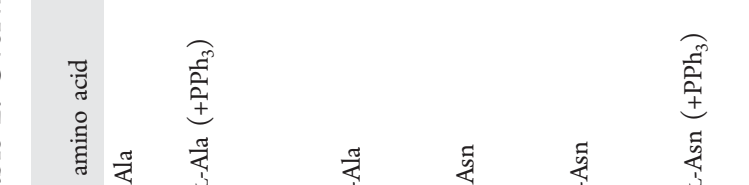

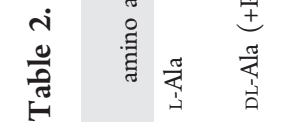

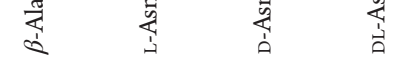

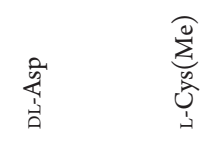

삽 숭 삽

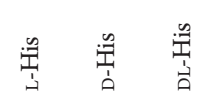


ions connect the $\mathrm{C}$-termini of the dipeptides via $\mathrm{Ag}-\mathrm{O}$ bonds, forming an eight-membered ring (Figure 3). Even for peptides

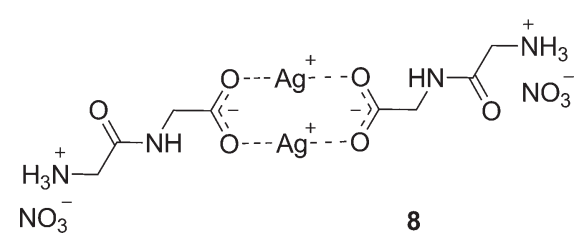

Figure 3. Schematic representation of the silver-diglycine complex 8 formed at $\mathrm{pH}=6$ according to Acland and Freeman. ${ }^{31}$

frequently used in combination with silver such as glutathione (GSH) (see section 2.2) or the AG4-peptide (see section 2.3.1), no crystal structure has been published. For glutathione, for example, there is only a proposed structure published for the GSH-silver complex, which is based solely on solid state ${ }^{13} \mathrm{C}$ nuclear magnetic resonance (NMR) and infrared (IR) spectroscopy. ${ }^{48}$ Besides, a recent review on metal-peptide frameworks does not describe such structure in combination with silver, ${ }^{49}$ underlining the difficulty in obtaining suitable crystals.

Even though there is not so much known about the detailed coordination behavior of silver toward peptides, the variety of sites suitable for metal binding can be capitalized in mass spectrometry (MS) because the use of metal ions as cationizing agents is widespread. $\operatorname{Ag}(\mathrm{I})$ has been used for the characterization of peptides as well. Electrospray in combination with collision-induced dissociation (CID) of $\mathrm{Ag}(\mathrm{I})$ complexes of amino acids and smaller peptides revealed, for instance, common fragmentation pathways, yielding predominantly $\mathrm{N}$ terminal ions, which is in contrast to the behavior of protonated peptides normally present using standard conditions for MS. ${ }^{50-52}$ However, due to the formation of doubly charged ions resulting from the complexation of $\mathrm{Ag}(\mathrm{I})$ and a proton, the fragmentation pattern and its interpretation are rather complicated. This problem was solved using matrix-assisted laser desorption/ionization (MALDI) in combination with CID, which allowed for the sequencing of larger peptides and proteins and might in the future also be used for the de novo sequencing of peptides and proteins. ${ }^{53}$ The analysis of nonfragmented peptides complexed with silver is possible by means of MALDI time-of-flight (TOF) MS. Through the addition of trifluoroacetic acid (TFA) to the sample, the number of $\mathrm{Ag}(\mathrm{I})$ complexes can be reduced as compared to the TFA-free solution due to the protonation of the basic amino acid residues, thus providing additional information, ${ }^{54}$ but not necessarily reflecting complexes formed at biological $\mathrm{pH}$.

Besides MS, $\operatorname{Ag}(\mathrm{I})$ is also employed as an analytical tool in other spectroscopic fields. Interestingly, it can be used to selectively detect the level of cysteine in biological fluids, although in theory Cys is not among the amino acids with a high silver ion affinity (Table 1). ${ }^{55}$ This detection is of great interest because the amount of cysteine present in physiological samples was found to be an important marker for clinical disorders. ${ }^{56,57}$ The addition of $\mathrm{Ag}(\mathrm{I})$ to a cysteine-containing solution leads to a self-assembly of chiral complex nanoparticles consisting of $\operatorname{Ag}(\mathrm{I})$ and cysteine. The formation of these particles results in a significant change in the circular dichroism (CD) spectra, which is not observed for the other naturally occurring amino acids or related biologically relevant thiols such as glutathione or homocysteine. Therefore, this CD method seems to be a promising probe for cysteine, working selectively and at a micrometer level. ${ }^{55}$ These results were recently confirmed by an independent work of Jiang and coworkers, ${ }^{58}$ focusing on a mechanistic analysis. They could show that interactions between the silver atoms, the so-called argentophilic attractions, can be switched on and off depending on the $\mathrm{pH}$ resulting in a decrease of the $\mathrm{CD}$ signal with increasing $\mathrm{pH}$ as a consequence of the loss of the argentophilic attraction due to the electrostatic repulsion of the carboxylates present at high $\mathrm{pH}$ values. ${ }^{58}$

A strong interaction between silver ions and cysteine was also reported by Feng at al. ${ }^{59}$ for their on-off sensor for silver ions and cysteine based on desoxyribonucleic acid (DNA)-functionalized gold nanoparticles. In this system, cysteine is able to remove $\mathrm{Ag}(\mathrm{I})$ from the cytosine- $\mathrm{Ag}(\mathrm{I})$-cytosine base pair complex (off-signal) that was responsible for a DNA duplex formation detected by light scattering (on-signal). ${ }^{59}$

As a concluding remark, the interaction of cysteine with ionic silver seems to be underestimated if taking into account only the binding affinities presented in Table 1 . However, its role should also not be overrated as the only important binding site in peptides and proteins because other amino acids such as histidine are excellent silver binding sites as well. After this overview of the interaction of silver ions with simple amino acids and short peptides, the following sections will focus on natural peptides and their role in metal detoxification and peptide-mediated formation of silver nanostructures.

\subsection{Natural Peptides Involved in Metal Detoxification}

To the best of the authors' knowledge, $\operatorname{Ag}(\mathrm{I})$ is not essential for biological processes. However, nature has to deal with silver ions present either naturally in the environment or due to the widespread use of silver, for example, in the photographical industry or medicine. Concerning the toxicity of silver, especially its accumulation is important, which is more often found in aquatic ecosystems than in their terrestrial counterpart as is typical for heavy metals. ${ }^{60}$ Therefore, bacteria and higher organisms had to develop resistance mechanisms to render $\mathrm{Ag}(\mathrm{I})$ and other toxic metal ions innocuous. Some bacteria developed a rather complex resistance machinery that also involves the use of silver binding proteins, which will be discussed in detail in section 3.2. Many organisms respond to heavy metal stress with the synthesis of binding proteins such as metallothioneins (MT), which can complex the harmful metal ions. ${ }^{61}$ They are ubiquitous and thought to be important for a wide range of biological functions such as transport, storage, and, as just mentioned, detoxification of essential and nonessential trace metals. After giving a short introduction of this class of low molecular weight proteins, whose affinity to silver is well-known, ${ }^{62}$ we will only focus on the work related to $\operatorname{Ag}(\mathrm{I})$.

The first MT was discovered in 1957, when this name was given to a metal-containing cysteine-rich protein, isolated from equine renal cortex. ${ }^{63}$ By definition, members of this family of proteins (i) are of low molecular weight, (ii) possess a high metal and sulfur content, (iii) have a characteristic amino acid composition, which is cysteine-rich but lacks the aromatic amino acids, and (iv) coordinate to metal ions through mercaptide bonds, due to the fact that all Cys residues are present in their reduced state, which gives rise to characteristic spectroscopic features of metal-thiolate clusters. ${ }^{64}$ These characteristics can be found in numerous peptides and proteins in many organisms. Therefore, the MTs are subdivided into 
three classes, with polypeptides in class I having cysteine locations closely related to those in the first reported MT; members of class II, the biggest class of MTs, having locations of cysteins only distantly related to those in equine MT; and class III comprising metal-thiolate polypeptides that are not genetically coded. ${ }^{69}$ In their metal-free (apo) form, the MTs lack almost any secondary structural elements. Therefore, an ordered spatial structure is only observed in the metalcontaining (holo) form induced by the presence of metal ions. Among others, this is one reason why three-dimensional structures of MTs are rare, no matter if taking into account computed or crystal structures. ${ }^{66}$

Because $\mathrm{Ag}(\mathrm{I})$ is NMR-active, it is discussed as a good probe for $\mathrm{Cu}(\mathrm{I})$ in the structure determination of $\mathrm{Cu}$-containing peptides and proteins. However, structural differences might arise from silver's larger ionic radius ${ }^{67}(\mathrm{Ag}(\mathrm{I}) 137 \mathrm{pm}, \mathrm{Cu}(\mathrm{I})$ $77 \mathrm{pm}$ ) and from $\mathrm{Ag}(\mathrm{I})$ preferring a linear over a trigonal planar (or tetrahedral) coordination geometry as preferred by $\mathrm{Cu}(\mathrm{I})$.

There is so far no silver-containing crystal structure of MTs reported in the literature and only one solution structure for yeast-MT based on NMR data, which is shown in a simplified way in Figure $4 .{ }^{68}$ Peterson et al. ${ }^{68}$ published this theoretical

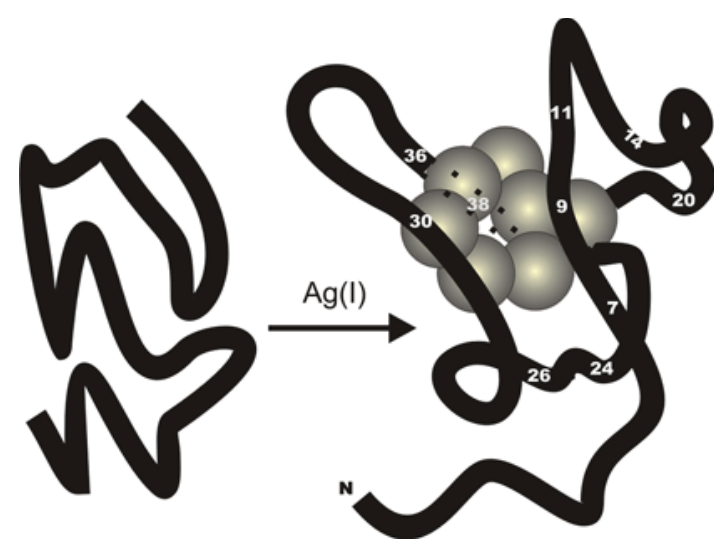

Figure 4. Schematic representation of the solution structure of the truncated $\mathrm{Ag}(\mathrm{I})$-coordinated yeast metallothionein, which lacks structural motifs in its apo-form. The numbers indicate the position of the 10 Cys-residues that are responsible for silver binding that is, in turn, the driving force for the protein folding. Adapted with permission from ref 68. Copyright 1996 Elsevier.

structure for a truncated yeast-MT (the flexible C-terminal end was excluded from the calculation) resembling a sprung paperclip, with the peptide backbone folding around a cluster of seven $\mathrm{Ag}(\mathrm{I})$. It forms two parallel loops that are separated by a deep cleft containing the metal cluster. This structure is very similar to the analogue $\mathrm{Cu}(\mathrm{I})$ species, which has been calculated as well. For the $\mathrm{Cu}(\mathrm{I})-\mathrm{MT}$, there are only slight contractions and a more flexible $\mathrm{N}$-terminus. The latter is probably due to the lack of N-terminal hydrogen bonding. Additionally, there is evidence that the coordination of one of the metal ions is different in both structures with Cys $9^{69}$ coordinating only in the copper-containing protein. However, this is an encouraging example for the structure prediction of $\mathrm{Cu}(\mathrm{I})$ proteins with the help of $\mathrm{Ag}(\mathrm{I})$ derivatives.

The strong affinity of MTs toward metal ions has also been used in the fabrication of biosensors for various heavy metals, including the detection of $\mathrm{Ag}(\mathrm{I})$ in drinking water. In 2009, a potentiometric sensor was described, using $\operatorname{Ag}(\mathrm{I})$ selective electrodes. $^{70}$ This selectivity was achieved by the incorporation of MTs into the membrane with poly(arylether sulfone) as polymeric matrix. Besides $\mathrm{Hg}(\mathrm{II})$, the other tested metal ions caused no or merely minor interferences, and the limit of detection was estimated to be around $10 \mu \mathrm{M}\left(1079 \mu \mathrm{g} \mathrm{L}^{-1}\right)$. A few months later, Kizek and co-workers ${ }^{71}$ proposed an electrochemical biosensor for the detection of $\mathrm{Ag}(\mathrm{I})$ based on MTs adsorbed on the surface of a hanging mercury drop electrode. The detection limit for $\operatorname{Ag}(\mathrm{I})$ was estimated to be $0.5 \mu \mathrm{M}\left(53.9 \mu \mathrm{g} \mathrm{L}^{-1}\right)$, which is 20 -fold more sensitive than the matrix-bound MT sensor. Still, this device is not a high precision instrument, but it is rather cheap, easy to use, and sensible enough to detect $\mathrm{Ag}(\mathrm{I})$ in contaminated drinking water because the maximum allowed contaminant level in drinking water is approximately $1 \mu \mathrm{M}\left(107.9 \mu \mathrm{g} \mathrm{L}^{-1}\right)$ depending on the country. ${ }^{72}$ Recently, the same group was able to present a new electrode with a 1000-fold lower detection limit of $0.5 \times 10^{-3}$ $\mu \mathrm{M}\left(53.9 \times 10^{-3} \mu \mathrm{g} \mathrm{L}^{-1}\right)$. They used a carbon paste electrode that has been decorated with MTs via anti-MT antibodies. ${ }^{73}$ This strong interaction might be a key factor for the low detection limit because the MTs are better captured on the electrode surface as compared to simple adhesion as in case of the hanging mercury drop electrode. As for the potentiometric determination described above, $\mathrm{Hg}$ (II) is the only ion interfering with the measurement, due to its higher affinity to MTs as compared to $\operatorname{Ag}(\mathrm{I}) .^{74}$ The main advantage for this system besides its low detection limit is claimed to be the easy miniaturization that could lead to the development of new working electrodes out of carbon nanomaterials immobilized with MTs. $^{73}$

Another important type of peptides in terms of metal detoxification is phytochelatins (PC), which are classified as class II MTs. ${ }^{75,76}$ These enzymatically synthesized peptides are produced by plants upon the exposure of the cells to toxic metals including silver. Such metal ions activate the PC synthase that catalyzes the conversion of GSH to PC (Figure $5)$. Their general structure is $(\gamma \text {-Glu-Cys })_{n} \mathrm{X}$, with $\mathrm{X}$ being Gly

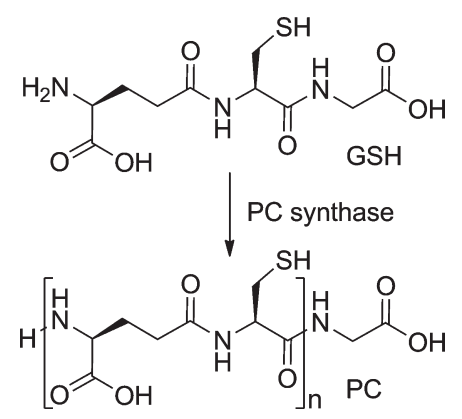

Figure 5. The enzymatic synthesis of PC starting from GSH.

(PCs), $\beta$-Ala, Ser, or Glu (iso-PCs) and $n$ commonly being in the range between 2 and 4 , but also higher values (up to $n=11$ ) have been reported. ${ }^{75}$ The PCs function as potent complexing agents in the cytosol of the cell and in this way preserve the cell from damage due to the heavy metal ions. Recent electrochemical studies ${ }^{77}$ confirmed that the amount of thiols such as phytochelatin and glutathione produced by plant embryos is proportional to the $\mathrm{Ag}(\mathrm{I})$ concentration and the time of exposure. This is in agreement with the current understanding that these molecules are part of the plant's defense system against heavy metals, ${ }^{77}$ in which the PCs-metal complexes are transported into vacuoles ${ }^{78}$ where the metal ion is less harmful. 
Recently, there has been a growing interest in glutathione itself due to its ability to form stable hydrogels together with $\mathrm{Ag}(\mathrm{I})$ depending on the silver/thiol ratio. ${ }^{79,80}$ The selfassembly of both components is proposed to lead to the formation of a polymeric network, stabilized by $\mathrm{Ag}(\mathrm{I})-\mathrm{Ag}(\mathrm{I})$ attractions, which were already discussed for the crystal structures of silver-amino acid complexes (see section 2.1.2), and additional noncovalent interactions such as hydrogen bonds as evidenced by Fourier-transform (FT) IR. The socalled argentophilic gel is stable in the $\mathrm{pH}$ range from 2 to 5 and can be converted reversibly into a clear solution through addition of an acid or a base, respectively, and can therefore be assigned as $\mathrm{pH}$-responsive. The interaction with $\mathrm{Ag}(\mathrm{I})$ seems to be only based on the presence of the thiol function in GSH, whose stretching band is absent in the formed $\mathrm{Ag}(\mathrm{I})$ thiolate (GS-Ag).$^{79}$ One future perspective is the use of these hydrogels as drug delivery system with the thiol component being not GSH itself but a thiol-containing drug. As proof of principle, the mucolytic drug $N$-acetyl-L-cysteine was used for the formation of argentophilic hydrogels. ${ }^{81}$ Furthermore, GSHbased argentophilic hydrogels were recently reported to act as an iodide-responsive material with the iodine functioning as a depolymerizing agent ${ }^{82}$ or as a ratiometric luminescent sensor for the presence of $\mathrm{Ag}(\mathrm{I})$ using a modified GSH carrying an aromatic fluorophore. ${ }^{83}$ Another possible application of silverGSH hydrogels lies in the biomedical field. Tang and coworkers $^{84}$ showed that the addition of $\mathrm{Ca}$ (II) leads to crosslinking, thus decreasing the solubility of the gel in water, which is favorable for a slow release of silver as an antimicrobial compound. Indeed, they could show that after a fast initial silver release in buffered saline during the first hours, the release continues up to 6 days as it was determined by inductively coupled plasma (ICP) method. The release of silver ions is thought to be triggered by the leakage of the cross-linking $\mathrm{Ca}(\mathrm{II})$, leading to the gradual destruction of the hydrogel accompanied by silver ion release.

Both PCs and GSH have lately been used for the synthesis of AgNPs due to their good ability to encapsulate the growing particle, and thus terminating the growth of AgNPs. ${ }^{85}$ This capping is crucial for the stability of metal nanostructures in general. Moshe and Markovich, ${ }^{86}$ for example, used GSH for the preparation of hollow AgNPs, using GSH-stabilized silver oxide nanoparticles that were chemically reduced in a counter diffusion process. Recently, it was shown that GSH can be more than a simple capping agent because it can be used as an anchor for further protein modification of the stabilized AgNPs. Thus, $\mathrm{Wu}$ et al. ${ }^{87}$ successfully synthesized surface-modified AgNPs with the help of GSH (see section 2.3.1). The thiol group of GSH absorbs on the silver surface and its N-terminus serves as an anchoring group for a glutaraldehyde linker via the formation of a secondary imine. The second aldehyde function is used for the covalent attachment of a protein such as bovine serum albumin as a model compound. ${ }^{87}$ The use of PC as a capping agent was demonstrated, for example, by Kumar et al. ${ }^{88}$ for an enzymatic route toward AgNPs using the enzyme nitrate reductase for the silver reduction in combination with $\mathrm{PC}$ as the capping agent.

The general ideas presented in this section for peptides involved in metal detoxification will in the following section be extended to peptides in general, which were either discovered by affinity screenings or synthesized de novo.

\subsection{Peptides for the Formation of Silver Nanostructures}

As will be demonstrated in section 3.3 on the example of bacteria, it is known that microorganisms are able to form AgNPs. This process is embraced by the generic term "biogenesis of inorganic nanoparticles". It has already been reviewed in recent publications, ${ }^{89,90}$ as well as the work on protein and peptide-directed production of inorganic materials in general. ${ }^{91,92}$ The advantages in the use of peptides in these syntheses are among others the mild reaction conditions as compared to traditional procedures enabling the green synthesis of nanomaterials and the high control they offer over the size and shape of the formed material. ${ }^{91}$ This Review is focused exclusively on silver nanomaterials where either the peptides are used for its biomineralization and therefore are responsible for silver reduction, or they serve as structuredetermining scaffold and stabilizing agent while the reduction of $\operatorname{Ag}(\mathrm{I})$ to $\operatorname{Ag}(0)$ is performed by an additional reducing agent.

2.3.1. Biomineralization of Silver by Means of Peptides. The process of biomineralization comprises the nucleation, growth, and assembly of inorganic materials by biological systems. ${ }^{93}$ Examples for biomineralization-derived materials are among others the shells of diatoms (silicium dioxide), pearls and shells (calcium carbonate), as well as bones (hydroxyapatite $\left.(\mathrm{HA})=\left[\mathrm{Ca}_{10}\left(\mathrm{PO}_{4}\right)_{6}(\mathrm{OH})_{2}\right]\right)$. During the biomineralization process, peptides can function as a structural template controlling the size and shape of the formed material. ${ }^{91}$ When it comes to the peptide-mediated formation of silver nanostructures, the peptides may serve not only as scaffold, but as a reducing agent as well. A huge effort has been made to understand the mechanism of nanoparticle formation by microorganisms (see section 3.3). Nonetheless, even for one of the most prominent examples, Pseudomonas stutzeri (P. stutzeri) ${ }^{94}$ this process is still not fully understood. In addition, it is not always easy to identify the active biomolecule responsible for biomineralization produced by microorganisms or plants. These active biomolecules do not necessarily have to be peptides. With the extract of coffee or tea, for example, the AgNP formation is due to the presence of polyphenolic compounds like catechins or the xanthine alkaloid caffeine. ${ }^{95,96}$ However, it has been shown that peptides do play an important role in the formation of AgNPs. To identify the ones responsible for silver binding and precipitation, the phage display technique (Figure 6), which was developed in 1985 by Smith, ${ }^{97}$ had an enormous impact. ${ }^{98}$ Rodi and Makowski ${ }^{99}$ described the power of this technique called biopanning with the successful search for a needle in a molecular haystack. The haystack consists of a peptide library in which each peptide is displayed on the surface of a bacteriophage viron by inserting foreign DNA segments into an appropriate location within the phage genome. This physical linkage of the DNA encoding the displayed peptides later allows their identification and is the basis of this technique. This phage display library is incubated with the desired target, in our special case the surface of AgNPs, allowing the peptides to interact with the target. This selection step is also termed "panning". After all of the unbound phages are washed away, the bound ones are eluted by changing the $\mathrm{pH}$ and eventually amplified for an additional panning cycle improving the screening result. Their peptide sequence can then be determined by deciphering the inserted nucleic acid. Stone and co-workers ${ }^{100}$ demonstrated that for very strongly interacting peptides this standard procedure has to be adapted because these strong binders are not eluted within the acidic washing step and therefore would get lost. To overcome this 

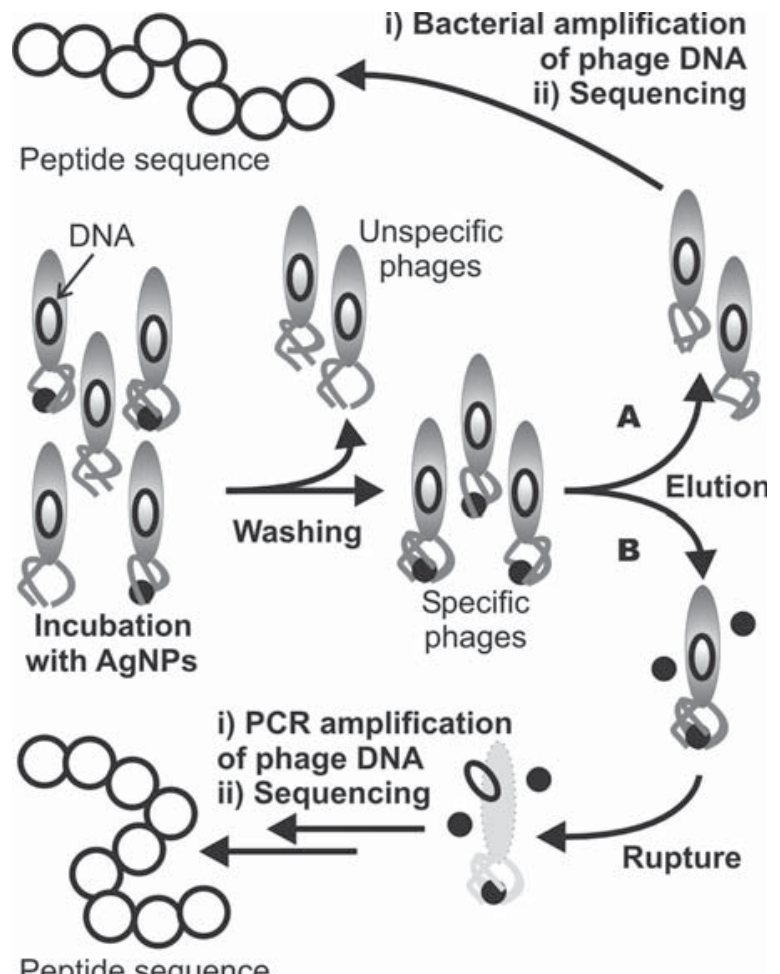

Peptide sequence

Figure 6. Schematic depiction of the standard biopanning method (path A) and the modified PCR-driven procedure (path B) for the sequence determination of silver-binding peptides. Adapted with permission from ref 100. Copyright 2004 Wiley-VCH Verlag GmbH \& Co. KGaA, Weinheim.

problem, they developed a novel screening method based on the polymerase chain reaction (PCR) schematically shown in Figure 6. This PCR-driven method allows the amplification of the phage DNA fragment including the region encoding the displayed peptide, whose amino acid sequence can be determined by DNA sequencing. The improvement obtained by the PCR-driven screening was demonstrated on the screening of a library for the detection of silver-binding peptides. While with the regular panning only three peptides could be identified, ${ }^{101}$ the improved screening located 14 additional peptides capable of silver-binding and reduction. ${ }^{100}$ The use of rolling cycle amplification is supposed to further improve the results of the phage display technology because it allows for the direct amplification of phage DNA while it is still bound to the substrate. ${ }^{102}$

One of the best studied examples of the silver-binding peptides detected by biopanning is the dodecapeptide Asn-ProSer-Ser-Leu-Phe-Arg-Tyr-Leu-Pro-Ser-Asp named AG4 that was discovered by Naik et al. ${ }^{101}$ Its three-dimensional chairlike structure was predicted with the help of NMR data, and the amino acids Leu5, Phe6, and Arg7 were found to be the ones whose NMR signals were most shifted upon the addition of $\mathrm{Ag}(\mathrm{I}) .{ }^{103}$ It was therefore concluded that those residues are responsible for the interaction with silver. This example shows that calculated binding affinities for amino acids (Table 1) are not necessarily confirmed in peptides. Nowadays, it is well known that peptides targeting inorganic materials such as silver are often able to mineralize the targeted elements as well, forming nanostructures. Naik et al. ${ }^{101}$ showed not only that the AG4 peptide is capable of reducing $\mathrm{Ag}(\mathrm{I})$ yielding, according to an analysis by transmission electron microscopy (TEM),
AgNPs with sizes of $60-150 \mathrm{~nm}$ and hexagonal, spherical, or triangular shapes, but they demonstrated further that this peptide can be used for the generation of silver crystal arrays on a glass surface using micromolding in capillaries. The interaction of the AG4 peptide with silver also gives rise to other applications. One example was recently reported where the AG4 peptide in connection with AgNPs was used for Raman spectroscopic conformational studies of large proteins interacting with silver surfaces. ${ }^{104}$ For this purpose, the AG4 peptide was fused to the C-terminus of a protein that itself shows only little affinity to AgNPs used as surface enhancers for Raman spectroscopy. Such a short terminal extension is normally well tolerated, and in this case increases the binding and orientation of the protein, thus improving the quality of the obtained data. This method might be useful for the investigation of peptide conformation and orientation on other surfaces used in biotechnological applications, for example, aluminum or platinum. ${ }^{104}$ A second example for the use of the AG4 peptide is the fabrication of silver nanowires. For this purpose, the encoded AG4 peptide was genetically fused to the gene of a protein acting as scaffold. ${ }^{105}$ After expression and purification, the scaffold proteins self-assemble, induced by guanosine-5'-triphosphate, into nanometer-scale fibers, decorated with the silver reducing AG4 peptide. Incubation with $\mathrm{Ag}(\mathrm{I})$ resulted in almost completely silvercoated nanowires. Authors claim that with optimized coating conditions the fabrication of continuous silver-coated nanowires should be possible. ${ }^{105}$

Another peptide capable of producing AgNPs was identified by Sano et al. ${ }^{106}$ They reported an interesting cross selectivity for a titanium-binding dodecapeptide (TBP-1) with the primary structure Arg-Lys-Leu-Pro-Asp-Ala-Pro-Gly-Met-His-Thr-Trp, which was discovered by means of biopanning as well. Out of 10 different metals, TBP-1 was able to mineralize silver in addition to silicium and titanium, forming AgNPs with a size in the range of $300-500 \mathrm{~nm}$ and a rectangular shape. For the formation of silver, silica, and titanium, the three amino acids Arg1, Pro4, and Asp5 are essential because the substitution of either one of them by alanine results in nonactive peptide mutants. ${ }^{106}$ It should be kept in mind that mutation experiments provide a first insight into the binding mechanism, but for a deeper understanding, for example of conformational changes upon silver binding, NMR studies or single-crystal Xray diffraction are able to provide more detailed information.

Very recently, the first in vivo study using engineered metalbinding peptides was published in which Escherichia coli (E. coli) secretes a short metal binding protein (Glu-Gln-LeuGly-Val-Arg-Lys-Glu-Leu-Arg-Gly-Val that was before identified by biopanning) either into the periplasm or into the cytoplasm. ${ }^{107}$ Interestingly, the strains possessing the periplasmic version of the peptide were able to survive in the presence of ionic silver $\left(28 \mu \mathrm{M}, 3020 \mu \mathrm{g} \mathrm{L}^{-1}\right)$, while the ones with the peptide located in the cytoplasm showed no resistance. Because AgNPs located in the periplasm were observed by TEM analysis for the silver-tolerant strain, it is proposed that the peptides are involved in the accumulation and reduction of ionic silver, thus forming less soluble and therefore less toxic NPs. ${ }^{107}$ This purely peptide-mediated resistance has so far not been reported for natural systems, but it could be an additional resistance mechanism to the ones presented in section 3.2.

The number of organisms and with that the number of peptides that are known to be able to produce nanosized silver is still growing, but the fundamental principles behind this 
fascinating process are still not understood to a satisfactory level. Even though many studies only described the phenomenon of silver reduction by means of peptides and did not go for a more detailed mechanistical analysis of this process, there soon was an agreement on the fact that the size and shape of the formed nanostructures are influenced by the peptide sequence.

The Role of Tyrosine. Xie et al. ${ }^{108}$ performed one of the most detailed studies of a biological AgNP synthesis by analyzing the extract of the green algae Chlorella vulgaris (C. vulgaris), which is capable of forming silver nanoplates. They first identified algal proteins to be the active biomolecules in this extract because under denaturing conditions, for example, heat or urea they found smaller nanoplates as compared to the nonmodified extract (average size of $27 \mathrm{~nm}$ versus $44 \mathrm{~nm}$ ). With only the size being influenced, the conclusion was that upon denaturation more amino acids become accessible for the interaction with silver. They went one step further and tried to identify the key players among the amino acids responsible for silver interaction and reduction. By Fourier transform (FT) IR, fluorescence analysis, and the use of acetylated derivatives, they could show that Tyr residues are responsible for the silver reduction by these algal proteins. ${ }^{108}$ Earlier, Selvakannan et al. ${ }^{109}$ already reported that tyrosine is able to reduce $\operatorname{Ag}(\mathrm{I})$ under alkaline conditions due to the deprotonation of the phenolic group and proposed a semiquinone as its final oxidation product. ${ }^{110}$ Xie et al. ${ }^{108}$ modified this model for neutral tyrosine with the first step being the coordination of $\operatorname{Ag}(\mathrm{I})$ acting as a Lewis acid and binding to the hydroxyl group. In total, one tyrosine is claimed to reduce two $\operatorname{Ag}(\mathrm{I})$ generating two protons and the semiquinone with a neutral tyrosyl radical generated as an intermediate. ${ }^{108}$ These stable radicals, formed upon the loss of a proton and an electron, are well-known intermediates in electron transfer processes, for example, in natural systems such as in the enzyme ribonucleotide reductase as the most prominent example $\mathrm{e}^{111}$ or have been detected in designed model peptides used for the determination of rate constants of electron transfer steps. ${ }^{112}$ The tyrosyl radical can therefore be considered as a probable intermediate involved in the reduction of $\operatorname{Ag}(\mathrm{I})$ as well. However, the semiquinone 9a being the proposed final oxidation product of tyrosine $(\mathbf{9 b})$ as depicted in the literature ${ }^{108-110}$ is not an oxidized form of tyrosine but one of its tautomeric forms and therefore cannot be the final oxidation product upon silver reduction (Figure $7 \mathrm{a}$ ). Because the oxidation of $p$-cresol (10) by cytochrome $\mathrm{P}_{450}$ was shown to lead to the formation of a very reactive quinone methide intermediate 11, which can then be trapped by glutathione to yield the GSH-conjugate $\mathbf{1 2}$ as shown in Figure $7 \mathrm{~b}$, a similar intermediate might be discussed for the oxidation of tyrosine. ${ }^{113}$ However, this tyrosine analogue of $\mathbf{1 1}$ was to the best of our knowledge only once reported in the literature. ${ }^{114}$ For further insight in the tyrosine-mediated reduction of $\mathrm{Ag}(\mathrm{I})$, the work on gold nanoparticle formation has to be taken into account. Mandal and co-workers ${ }^{115}$ postulated that starting from a tyrosinate-containing peptide 13 , a neutral radical 14 as an intermediate is formed, which was detected by electron paramagnetic resonance (EPR) spectroscopy. This radical recombines with a second tyrosine radical to form dityrosine (15) as tyrosine's final oxidation product (Figure 7c), which in turn can be detected easily through its emission peak at around $410 \mathrm{~nm}$. In proteins, such formation of dityrosine leads to a) Tyrosine tautomerism<smiles>[R]c1ccc(O)cc1</smiles>

b) Oxidation of $p$-cresol

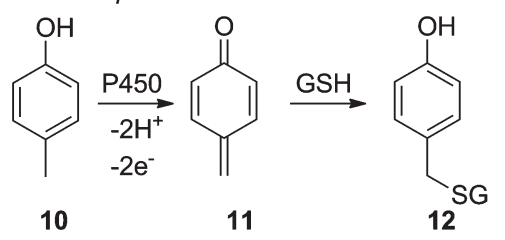

c) Dityrosine formation<smiles>[R]c1ccc(-c2c(O)cc([R])cc2-c2cc([R])ccc2O)cc1</smiles>

Figure 7. (a) Two possible tautomeric forms of tyrosine. (b) Oxidation of the related $p$-cresol (10) leads to the quinone methide intermediate 11 trapped with GSH $(\rightarrow 12)$. (c) Dityrosine formation (15) as a possible oxidation pathway of tyrosine.

cross-linking of peptides, which can be either a normal process or a pathological response to disease or oxidative stress. ${ }^{116}$

An analogue redox process is likely involved in the formation of AgNPs as well. Both, Si et al. ${ }^{115}$ and Xie et al. ${ }^{108}$ reported that the tyrosine content in tripeptides as model compounds for larger proteins influences the rates of silver ${ }^{108}$ or gold ${ }^{115}$ nanoparticle formation. Another important factor influencing the rate of tyrosine-mediated mineralization is the $\mathrm{pH}$ value of the reaction medium as it was, for example, shown for tyrosineand ruthenium-containing model compounds mimicking the photosystem II, where they proposed a proton coupled electron transfer for tyrosine $(\mathrm{pH}<10)$ and a faster single electron transfer step starting from tyrosinate $(\mathrm{pH}>10) .{ }^{119}$ The same trend was observed by Mandal and co-workers ${ }^{115}$ for the formation of gold nanoparticles by means of tyrosinecontaining peptides. Xie et al. ${ }^{108}$ did not study the influence of the $\mathrm{pH}$ on the AgNP formation but discussed the influence of the silver-peptide complex formation on the redox potential of the $\operatorname{Ag}(\mathrm{I}) / \operatorname{Ag}(0)$ couple showing that complexation generally lowers the redox potential and therefore facilitates the reduction of $\operatorname{Ag}(\mathrm{I})$ (see Table 3). They underlined their

Table 3. Standard Electrode Potential $E^{\circ}$ in Volt for Different Silver Compounds

\begin{tabular}{lllll}
\multicolumn{1}{c}{ red } & $\rightleftharpoons$ & \multicolumn{1}{c}{ ox } & $+z \mathrm{e}^{-}$ & $E^{\circ}$ \\
$\mathrm{Ag}($ bulk $)$ & $\rightleftharpoons$ & $\mathrm{Ag}^{+}$ & $+\mathrm{e}^{-}$ & 0.80 \\
$2 \mathrm{Ag}+2 \mathrm{OH}^{-}$ & $\rightleftharpoons$ & $\mathrm{Ag}_{2} \mathrm{O}+\mathrm{H}_{2} \mathrm{O}$ & $+2 \mathrm{e}^{-}$ & 0.34 \\
$\mathrm{Ag}+\mathrm{Cl}^{-}$ & $\rightleftharpoons$ & $\mathrm{AgCl}$ & $+\mathrm{e}^{-}$ & 0.22 \\
$\mathrm{Ag}+\mathrm{Br}^{-}$ & $\rightleftharpoons$ & $\mathrm{AgBr}$ & $+\mathrm{e}^{-}$ & 0.07 \\
$\mathrm{Ag}$ & $\rightleftharpoons$ & $\mathrm{Ag}+\mathrm{Ag}^{+}$ & $+\mathrm{e}^{-}$ & -0.10 \\
$\mathrm{Ag}+\mathrm{I}^{-}$ & $\rightleftharpoons$ & $\mathrm{AgI}$ & $+\mathrm{e}^{-}$ & -0.15 \\
$\mathrm{Ag}$ & $\rightleftharpoons$ & $\mathrm{Ag}_{2}+\mathrm{Ag}^{+}$ & $+\mathrm{e}^{-}$ & $-0.95^{a}$ \\
$\mathrm{Ag}$ (atom) & $\rightleftharpoons$ & $\mathrm{Ag}^{+}$ & $+\mathrm{e}^{-}$ & -1.80
\end{tabular}

${ }^{a}$ Estimated from ref 119. 
statement with experiments using a series of tripeptides all containing a $\mathrm{C}$-terminal tyrosine and the two remaining amino acids bearing more or less efficient silver coordination sites. Indeed, for the tripeptides containing strongly coordinating amino acids such as histidine and cysteine they observed no AgNP formation, for peptides containing weakly coordinating amino acids, for example, lysine or aspartic acid the mineralization of silver was slow, while it was fast for noninteracting amino acid containing peptides such as glycine. $^{108}$ From these results, it was concluded that the complex has to be broken prior to silver reduction by tyrosine. Interestingly, these results imply that cysteine is a better ligand for $\operatorname{Ag}(\mathrm{I})$ than lysine, which is not in agreement with the calculated binding affinities discussed previously in section 2.1.1 (see Table 1). A similar system was investigated by Belser et al. ${ }^{118}$ who observed that a tripeptide containing both tyrosine and histidine as a strong silver binder is able to mineralize silver only after irradiation. The above summarized results for the involvement of tyrosine in the reduction process of silver clearly indicate that there is still a long way to go to understand the mechanistic pathways involved in the mineralization of silver, and it has to be seen whether the formation of dityrosine takes place in response to the presence of silver ions.

The Role of Aspartic and Glutamic Acid. Besides Tyr, Xie et al. ${ }^{108}$ identified Asp and/or Glu residues as key players in the peptide-mediated silver mineralization with regard to the shape of the formed particles. They found that their carboxyl groups present in the side chain are promoting the anisotropic growth and therefore dictating the shape of the formed nanostructures. This is based on an experiment where all of the carboxylic groups of the algal proteins were converted into amides, yielding predominantly spherical nanoparticles or irregularly shaped structures upon incubation with silver nitrate instead of triangular plates, which were found before. ${ }^{108}$ The role of glutamic acid was studied in greater detail by Fischer and coworkers ${ }^{120}$ who explored the effect of the peptide chain length of oligopeptides $(\mathrm{Glu})_{n}$ on the crystal growth of calcium oxalate and showed a dependence of the crystal morphology, phase, and stability on the peptide chain length. Nam et al. ${ }^{121}$ proposed that the amino acids with carboxylic side chains are also important for the reduction of $\mathrm{Ag}(\mathrm{I})$ by means of peptides in combination with ambient light. They postulated a mechanism for AgNP synthesis using engineered yeast decorated with hexaglutamic or hexaaspartic acid in which the coordination of silver ions by the carboxylic groups is supposed to lower the energy barrier for their reduction. ${ }^{121}$ This is at first glance contradictory to the results of Xie et al. ${ }^{108}$ discussed above where the complexation slowed the rate of AgNP formation, hence having a negative effect on the silver reduction. Nam and co-workers ${ }^{121}$ argued that the enhanced reducibility of silver ions due to the complexation with carboxyl groups is probably a cooperative effect. The complexation lowers the energy barrier for the reduction as compared to an unbound silver ion (see Table 3), which should be considered as starting point. In case of the hexaglutamic acid derivative, the pocket-like structure of the peptide leads to an increased local concentration of silver ions, which is also beneficial for the reduction process. Furthermore, the Fermi level of the silver cluster is lowered due to the electron donation of the nucleophilic carboxyl group (Figure 8), ${ }^{119}$ thus rendering the further reduction of $\mathrm{Ag}(\mathrm{I})$ more facile. ${ }^{121}$

Recently, Roy and Banerjee ${ }^{122}$ reported that Fmoc-protected phenylalanine forms a stable hydrogel capable of reducing silver

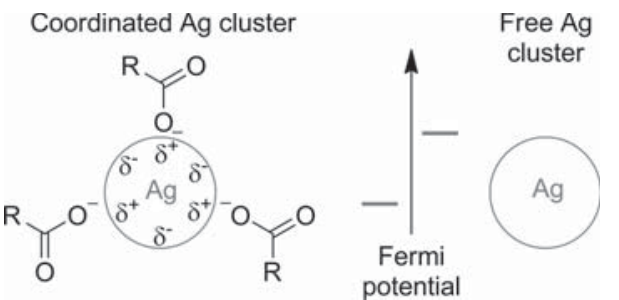

Figure 8. Influence of coordination of carboxylic acids on the Fermi potential of silver clusters.

spontaneously in the presence of light, forming $\mathrm{Ag}_{4}$ nanoclusters. Their formation is ascribed to the complexation of $\mathrm{Ag}(\mathrm{I})$ via the $\mathrm{C}$-terminal carboxylic acid together with the presence of light, which is similar to the above-described role of glutamic and aspartic acid. The latter has a large Stokes shift together with a narrow emission band, emitting red light, which might be interesting for the application in sensors. ${ }^{122}$ Also, Adhikari and Banerjee ${ }^{123}$ reported that in a short peptide-based hydrogel, the light-induced formation of silver nanowires is possible due to the combination of a carboxylic acid-containing peptide (Fmoc-Val-Asp-OH) and sunlight. ${ }^{123}$ These observations are in agreement with the results reported by Belser et al. ${ }^{118}$ who showed that a tetrapeptide containing Asp and Ser linked by a rigid linker that does not spontaneously form AgNPs upon incubation with silver nitrate is able to mineralize silver only in the presence of light. The suggested influence of the complexation with a peptide on the redox potential of silver is discussed controversially since the work of Carter et al. ${ }^{124}$ showed that the silver redox potential does not change in the presence of a glutamate hexamer. Nam and co-workers ${ }^{121}$ showed as well that silver nanowires can be produced through spontaneous photoreduction working with a modified M13 bacteriophage called E4 because it has tetraglutamate (EEEE) fused to the $\mathrm{N}$-terminus of each major coat protein ( $\mathrm{p} 8$ ). The M13 bacteriophage is one of the viruses commonly used in the phage display technique described above whose coat consists of about 2700 copies of the 50 amino acid long p8. The soobtained silver nanowires were further tested for their electrochemical behavior toward the use as anode in lithium ions batteries, serving as model system at the nano scale. ${ }^{125}$ For amino acids with side chains containing carboxylic acids, it can be concluded that they function as a complexation site for silver while their involvement in the silver reduction is discussed controversially. Nevertheless, a lot of work has to be done to achieve a better understanding and to allow for predictable shapes of the formed AgNPs.

The Role of Tryptophan. Like tyrosine, tryptophan is known to be important in biological electron transfer reactions involving DNA photolyase, ribonucleotide reductase, or azurin. ${ }^{126}$ Mandal and co-workers ${ }^{127}$ showed that tryptophancontaining peptides can indeed function as reducing agent for the formation of silver as well as gold nanoparticles. Similar to the work on tyrosine-containing peptides, ${ }^{115}$ they proposed a reaction under alkaline conditions, where the indole $\mathrm{NH}$ group is deprotonated (16), and the neutral tryptophyl radical (17) is formed as an intermediate, which can either dimerize to yield ditryptophan (19), reverse back to its native form, or form the kynurenine form (18) of the peptide (Figure 9). Kynurenine, a known metabolite of tryptophan, and ditryptophan are both highly fluorescent, which was useful for their detection during the nanoparticle formation. ${ }^{127}$ More evidence for the reducing power of tryptophan with regard to metal particle formation 


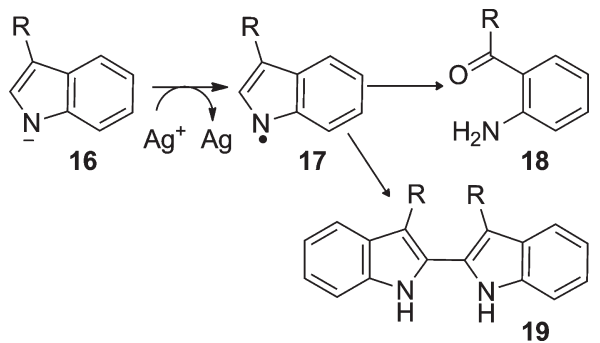

Figure 9. Possible oxidation pathways of tyrosine lead to the formation of kynurenine (18) and ditryptophan (19).

can be obtained looking at the formation of gold nanoparticles, where the oxidative polymerization of tryptophan was reported during nanoparticle formation. ${ }^{128}$

Other evidence for the importance of tryptophan was given by Carter et al. ${ }^{124}$ who investigated the silver mineralizing properties of a peptide previously isolated from a phage library designed for the evaluation of germania (germanium oxide) binders, ${ }^{129}$ which was therefore named Ge8. The formation of AgNPs with the help of these peptides additionally needed the presence of light and 2-[4-(2-hydroxyethyl)piperazin-1-yl]ethanesulfonic acid (HEPES) buffer because the formation of $\mathrm{H}_{2} \mathrm{O}_{2}$ as one involved reducing agent is accomplished via a photoinduced decomposition of the buffer. In addition to the photochemically formed reducing agent, the primary sequence of the peptide has an influence on the reduction process. Mutation experiments, where tryptophan or methionine, which, as sulfur-containing amino acid, can interact with silver ions as well, were exchanged against alanine, implement that both amino acids are necessary in case of $\mathrm{Ge} 8$ for silver reduction. ${ }^{124}$ In contrast, the exchange of histidine versus alanine results in the loss of silver ion affinity, but interestingly the formation of AgNPs is still possible with these mutants. ${ }^{124}$ In case of the $\mathrm{Ge} 8$, the peptide alone apparently cannot provide sufficient reducing power because a second reducing agent $\left(\mathrm{H}_{2} \mathrm{O}_{2}\right)$ is needed but seems to be more than just a scaffold for the shape control of the formed particles. The role of tryptophan in the particle growth of AgNPs was recently investigated by Kulesza et al. ${ }^{130}$ who proposed the doubly charged silver cluster $\mathrm{Ag}_{4}{ }^{2+}$ stabilized by tryptophan as an optical marker for particle growth due to its characteristic absorption spectrum. The possibility to monitor early stages of the AgNP growth might provide further insight into this process.

The Role of Sulfur-Containing Amino Acids. Even though tyrosine and tryptophan are discussed as the main reaction partners responsible for silver reduction, the participation of the two sulfur-containing amino acids cysteine and methionine cannot be ruled out completely. As briefly mentioned in the case of the $\mathrm{Ge} 8$ peptide, methionine is discussed to take part in the silver reduction process because mutants where this amino acids are substituted with alanine were not able to produce AgNPs. ${ }^{124}$ However, so far there is no experimental evidence whether this amino acid really serves as electron donor or has a different function in the complex procedure. Interestingly, cysteine alone was recently reported to be able to reduce silver ions in the presence of cetyltrimethylammonium bromide micelles. ${ }^{131}$ As mechanism, the formation of a silver-cysteine complex is proposed that is then able to reduce free silver ions and leads to the formation of a cysteyl radical. The micelle is thought to stabilize the substrate and the resulting complex and might in addition influence physical properties of the reactants. ${ }^{131}$ That the formation of a cysteyl radical is involved is underlined by the fact that at higher cysteine concentration the formation of a cystine silver complex was observed. ${ }^{131}$ Because the presence of micelles is needed for the silver reduction as well, we would not in general classify cysteine as a reducing amino acid by itself as is the case for tyrosine or tryptophan.

The Role of the Peptide Backbone and the Effect of Silver on the Secondary Structure. After having discussed the role of certain amino acid side chains in the AgNP formation by means of peptides, we will now briefly focus on the amide functional groups present in the peptide backbone because there is also some evidence that these groups are involved in the mineralization process as well. In general, it is known that the binding of peptides and proteins to nanoparticles can also occur over free amide groups, one of the main interactions between peptides and growing silver particles besides thioether bonding and interaction via benzyl groups of the peptides. ${ }^{132} \mathrm{Li}$ et al. ${ }^{133}$ were the first to use a plant extract (Capsicum annum L.) at room temperature for the green synthesis of AgNPs. They assigned its reducing capability to the amide groups of the proteins present in the plant extract. Unfortunately, this hypothesis is only underlined by IR and electrochemical data. In the IR, the plane bending vibration of the $\mathrm{NH}$-group in proteins is absent after silver reduction, indicating a relation between silver reduction and oxidation of the amide functions, while an oxidation peak present in the cyclic voltammogram of the plant extract shows its possible reducing power. ${ }^{133}$ Another example where the amides are believed to be responsible for silver reduction are the cyclic peptides curcacycline $\mathrm{A}^{134}$ and $\mathrm{B}^{135}$ (Figure 10), the major components in the latex of Jatropa curcas, which can be used for the generation of AgNPs as well. ${ }^{136,137}$

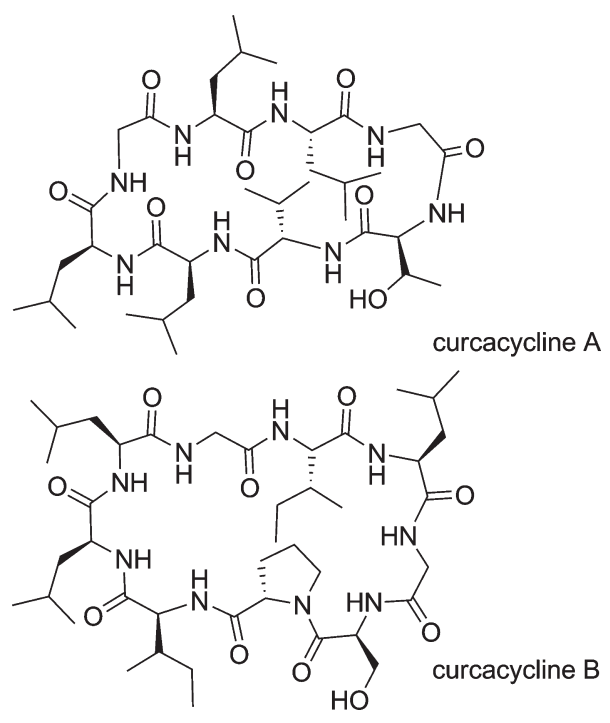

Figure 10. The two cyclopeptides curcacycline A and B are the major components of the latex of J. curcas.

Beside the role of the amide, $\mathrm{Li}$ et al. ${ }^{133}$ also investigated the influence of the redox process on the secondary structure of the peptide reporting a conformational disorder upon silver reduction because the percentage of $\alpha$-helix present in the structure decreased after the reaction. The same tendency of increased structural disorder was recently reported by Sanghi and $\mathrm{Verma}^{138}$ for the interaction of $\mathrm{Ag}(\mathrm{I})$ with fungal 
mycelium. The influence of the secondary structure on the formation of silver nanostructures was shortly discussed before for the algal proteins examined by Xie et al., ${ }^{108}$ which provided nanoplates with a different size upon denaturation of the proteins as compared to the nanoplates formed by the folded proteins. The change in the secondary structure seemed also to be one of the main factors involved in the AgNP synthesis catalyzed by the hen egg lysozyme, recently reported by Eby et al. ${ }^{139}$ A change of the solvent from water to methanol made the controlled growth of stable nanoparticles possible. While in water, silver was reduced by the native lysozyme, but colloidal stability could not be achieved, and in methanol, where the enzyme is denaturized, stable colloids were formed, which retained their stability to a high degree after dialysis into water. ${ }^{139}$ The use of lysozyme for the generation of AgNPs can also be important for medical applications because lysozome is an inhibitor of Gram-positive bacterial strains while AgNPs are active against both Gram-negative and Gram-positive strains. Indeed, Eby and co-workers ${ }^{140}$ introduced a system using the antibacterial activity of both lysozyme and AgNPs as possible coating for medical instruments (see section 4.1.5).

Conclusion. Having discussed different key factors involved in the reduction of $\mathrm{Ag}(\mathrm{I})$ by means of peptides and proteins, one bottom line is that even though huge effort has been undertaken to understand the mechanism behind this fascinating mineralization process, there is still a lot of work to be done to achieve a detailed insight into the mechanistic action. One of the current models is the concept of (i) recognition, (ii) reduction, (iii) limited nucleation, and finally (iv) growth of the silver particles. ${ }^{141}$ As demonstrated above, the interaction of peptides and proteins with silver seems to be important in all phases of the mineralization process, leading to numerous factors influencing the outcome of the reaction. With some of the amino acids such as Tyr and Trp being important for the reduction process, other groups such as amides or thioether containing residues seem to play a major role in terms of particle growth, while the amino acids with side chains containing carboxyl groups (Asp and Glu) are responsible for the silver coordination and nucleation and might be involved in the silver reduction process. One of the challenges to gain a better understanding of this multifunctional process is the difficult comparability and interpretation of the results present in the literature due to the use of peptides differing in length, structure, composition, polarity, and so on or, even more complicated, the use of extracts of natural products where a mixture of compounds is most likely involved in the formation of silver nanostructures. While the material scientists are more interested in the silver nanostructures, the fate of the oxidized peptides remains often unclear. This might however be of importance for the understanding of the biological activity of silver.

2.3.2. Peptides as Structure-Determining Scaffolds in the Synthesis of Silver Nanostructures. Peptides are known for their structural variety due to their ability to arrange the amino acids in secondary structural elements (helices, $\beta$ sheets, and turns) that in turn can form superior threedimensional arrangements. Because these structures are of a defined nature, they are good templates for the synthesis of materials of an ordered size and shape as they are capable of limiting the growth, preventing agglomeration, and organizing the AgNPs. Therefore, it is not surprising that they serve as the major biological scaffold material. ${ }^{142}$ The template effect is also used for the synthesis of different complex silver nanostructures as it will be shown in the following part on recent reports found in the literature. An array of AgNPs can for example be achieved using a self-assembled polymer-peptide conjugate as a matrix for the controlled particle growth. ${ }^{143}$ A poly(ethylene oxide)-peptide conjugate is reported to form stable doubletape constructs with a peptidic core consisting of $\beta$-sheets and a polymeric shell. These double-tapes stack together to finally form two-dimensional networks. The AgNPs are located in the peptidic core and are formed either upon irradiation of an $\mathrm{Ag}(\mathrm{I})$-containing solution or upon incorporation of preformed silver nanoclusters that aggregate in a controlled fashion inside the double-tapes. ${ }^{143} \mathrm{~A}$ second type of nanostructures obtained by using peptides as a structure-determining scaffold are metal nanowires, which are important for the development of new nanoelectronic materials. Gazit and co-workers ${ }^{144}$ showed that already small Phe-Phe dipeptides (20) derived from the $\beta$ amyloid polypeptide can be sufficient to self-assemble into peptide nanotubes, which can be used as scaffold for the synthesis of silver nanowires through reduction of the enclosed $\mathrm{Ag}(\mathrm{I})$ with sodium citrate. Subsequent enzymatic degradation of the peptidic template releases the metal nanowire (Figure 11). Instead of degrading the template, the reduced silver-filled

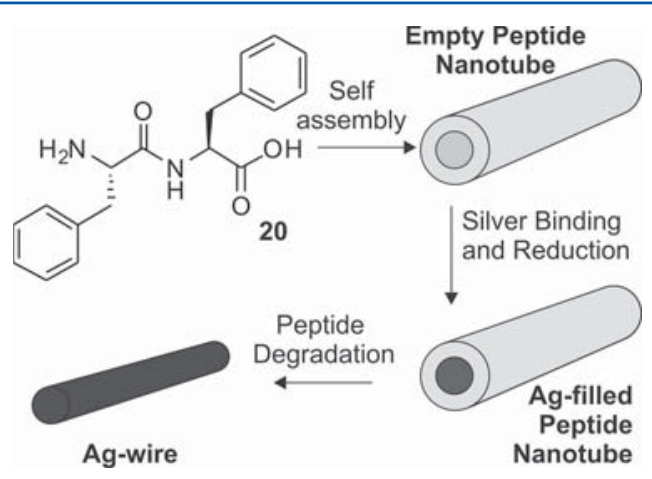

Figure 11. Schematic representation of the formation of silver nanowires using peptide nanotubes consisting of Phe-Phe dipeptides (20) as degradable template.

nanotubes can also be used for the synthesis of coaxial nanowires with a silver core and a gold outer shell. In this case, the intact peptidic template is decorated with a second peptide, functioning as a linker for the attachment of gold nanoparticles. These particles are used in the last step as nucleation sites for the gold deposition yielding a complex nanostructure with a coaxial geometry that might be used in nanotechnology. ${ }^{145} \mathrm{~A}$ third common target structure in nanochemistry are metal nanotubes. Bale et al. ${ }^{146}$ attached AgNPs to poly-L-lysine (PLL) or bovine serum albumin (BSA) functionalized multiwalled carbon nanotubes. After a 1-day exposure to a $\mathrm{Ag}(\mathrm{I})$-containing solution, the tubes were washed and the reduction of $\mathrm{Ag}(\mathrm{I})$ was accomplished using sodium borohydride leading to the formation of AgNPs on the surface of the nanotube. They also observed that the nanoparticle formation is hindered if the attached protein is glycosylated. These results indicated that the glycosylation state of proteins might be useful for the patterning of nanostructures. ${ }^{146}$ The same approach was also successful using active enzymes for the linkage between nanotube and nanoparticle yielding a multifunctional nanocomposite with the enzyme still being active. ${ }^{146}$

Peptides cannot only form ordered nanosized structures as just shown on some examples, but they are also able to form macroscopic assemblies such as hydrogels, which are well 
ordered on the nanometer scale. Taubert and co-workers ${ }^{132}$ reported the use of an organogel consisting of oligo-L-valine and a sulfur-containing peptide for the mineralization of silver using dimethylformamide as a reducing agent. The size and shape of the resulting AgNPs could be controlled by the ratio of the two gel-forming peptides, which is accompanied by the variation of the thiol to metal ratio demonstrating that the Brust-Schiffrin concept ${ }^{147}$ in which the size depends on the thiol amount is transferable to rather stiff supramolecular assemblies. $^{132}$ Oligovaline does not react with the formed AgNPs but is important for the gel formation, while the thiolcontaining peptide is necessary for silver coordination, nucleation, and organization.

Taubert and co-workers ${ }^{148}$ recently published an elaborate study on the influence of the peptide on the properties of the mineralized material, using the hexapeptide (Lys-Lys-Cys) ${ }_{2}$ in which the two subunits are dimerized via a disulfide linkage of the cysteine side chains. There has been strong evidence that the $\mathrm{pH}$ influences the aggregate formation of peptide-coated AgNPs due to different patterns of protonation/deprotonation with regard to the termini and the side chains of the amino acids. At low $\mathrm{pH}$, the hexapeptide was able to stabilize individual particles predominantly through interaction of the sulfur atoms with the AgNPs, while at high $\mathrm{pH}$ the formation of larger aggregates was observed, which might lower electrostatic repulsion with the lysine moieties being deprotonated. ${ }^{148,149}$ This aggregate formation, however, was reversible upon lowering the $\mathrm{pH}$ of the solution. They could further show that the obtained peptide-coated AgNPs can be coated with a silica shell retaining the chiral information carried by the coated $\mathrm{AgNPs}^{149}$ and that the incorporation of erbium into this shell leads to the formation of paramagnetic and chiral AgNPs. ${ }^{150}$

Recently, Upert et al. ${ }^{151}$ reported the use of oligoprolines as scaffolds for the size-controlled formation of AgNPs with the diameter of the AgNPs correlating with the length of the aldehyde-functionalized helical peptides. The aldehyde functions are used in a Tollens reaction to reduce the silver ions to AgNPs that are stabilized by the resulting carboxylic acids. Thus, the side-chain modification of peptides can offer additional possibilities to control the growth of AgNPs.

In this section, it was shown that peptides act as excellent scaffolds for the controlled synthesis of silver nanostructures, enabling the directed generation of, for example, nanotubes, nanowires, and arrays of nanoparticles. These nanostructures are interesting for technical and medical applications. Examples for the use of silver nanostructures in the construction of new materials will be discussed in section 4 , while the biocompatibility of silver and silver-functionalized materials will be reviewed in section 5. Prior to this, the following section will focus on the interaction of bacteria with silver. As indicated above, this interaction is not only important for the synthesis of AgNPs but also for the understanding of the antimicrobial activity of silver compounds.

\section{BACTERIA AND SILVER}

\subsection{Antimicrobial Properties of Silver-Containing Compounds}

It has been known for a long time that silver cations are highly toxic to bacterial cells. ${ }^{152-154} \mathrm{AgNO}_{3}$, for instance, shows an antimicrobial activity with a minimal inhibitory concentration (MIC) of $3 \mu \mathrm{M}\left(323 \mu \mathrm{g} \mathrm{L}^{-1}\right)^{155}$ against a silver-sensitive E. coli strain. Although silver cations have found extensive applications in various fields of industry and medicine (see sections 4 and 5), silver in its oxidation state zero has recently been of increasing interest as well. AgNPs have gained considerable attention over the last few decades in response to the raising need for antimicrobial compounds due to the increasing bacterial resistance to commonly used antibiotics. Because of their small size, nanoparticles possess new chemical, physical, and biological properties distinctive from those offered by traditional bulk materials. ${ }^{156}$ The particle size exhibits the general tendency that a smaller particle size results in a greater antimicrobial effect. This is related to the available surface area per unit mass, ${ }^{157,158}$ which allows a higher silver ion release and an easier interaction with other particles. ${ }^{159}$ Correspondingly, the formation of aggregates, leading to a reduced accessible surface area and thus reduced silver ion release, is probably responsible for a decreased antimicrobial activity. ${ }^{160}$ The investigation of the antimicrobial properties of AgNPs started at the beginning of the millennium, ${ }^{152}$ and they have since demonstrated their broad range of antimicrobial activity against Gram-positive as well as Gram-negative bacteria, ${ }^{152,157,158,161-166}$ fungi, ${ }^{163,164,166}$ and viruses. ${ }^{167-173}$

Silver compounds are reported to exhibit multidirectional activity to fight bacteria ultimately leading to cell death. Therefore, several attempts have been made to elucidate their mode of action. ${ }^{152,162,166,174,175}$ Some aspects of the antibacterial effect of silver nanomaterials were recently reviewed by Mariambio-Jones and Hoek. ${ }^{176}$ However, until present days, a complete understanding of the mechanism of silver ions and AgNPs fighting bacteria has not been reached. Nevertheless, there are several pathways known for the interactions between silver compounds and the bacterial cells. ${ }^{176}$ These interactions can be classified as direct interactions of the silver compounds with (i) the bacterial cell wall, (ii) DNA, (iii) enzymes and membrane proteins, and (iv) as interactions based on the silverinduced formation of reactive oxygen species (ROS). The interaction pathways are summarized in Figure 12 and will be discussed in more detail in the following. In addition, we will briefly focus on physical properties of AgNPs influencing their antimicrobial activity.

3.1.1. Interactions with the Bacterial Cell Wall. As shown in Figure 12, the interaction with the bacterial cell wall is one of the first points of attack and important for the

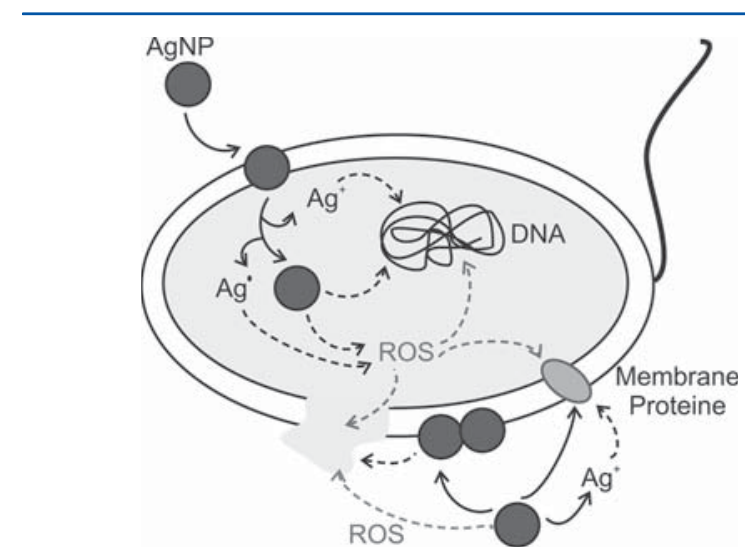

Figure 12. Possible interaction pathways of silver-containing compounds with bacterial cells: Direct interaction with (i) the cell wall, (ii) DNA, (iii) membrane proteins, and (iv) formation of ROS. Adapted with permission from ref 176. Copyright 2010 Springer Science and Business Media. 
antimicrobial activity because (i) the crossing of the plasma membrane allows silver compounds to interfere with the bacterial DNA located in the cytoplasm or to generate ROS in the interior of the cell (this will be discussed in the following subsections), and (ii) the accumulation of silver in the cell membrane affects its permeability, ${ }^{176}$ which can cause the leakage of the cytoplasm leading to cell death. Before discussing the interaction with the cell wall of bacteria, one should keep in mind that the bacteria are classified into two main groups depending on their cell wall structure. For Gram-positive bacteria, the cell wall consists of a thick peptidoglycan layer $(15-80 \mathrm{~nm}),{ }^{177}$ a polymer consisting of sugars and amino acids that forms a mesh-like structure on the outside of the plasma membrane. In addition, polyalcohols, so-called teichoic acids, are found in their cell wall providing additional stability. The peptidoglycan layer of Gram-negative bacteria on the outside of the plasma membrane is much thinner than that for Grampositive bacteria $(\sim 2 \mathrm{~nm}),{ }^{177}$ but they have an additional outer membrane composed of phospholipids and lipopolysaccharides around the peptidoglycan layer.

Using TEM, it has been shown that the direct interference of $\mathrm{Ag}(\mathrm{I})$ with bacterial cells causes morphological changes of their cell wall resulting in the detachment of the plasma membrane. ${ }^{175,178}$ Silver ions, for example, caused the lysis of Staphylococcus aureus (S. aureus) and E. coli cells by detaching the cell membrane from the cell wall resulting in the leakage of the cellular interior. On the TEM image, the presence of electron-rich silver-containing granules in the surrounding of the cell was observed, which is a clear indication for the interaction of silver ions with the plasma membrane. The observed effect was greater in case of Gram-negative E. coli than for Gram-positive S. aureus, which can be attributed to the above-described structural differences in their cell wall with the thick peptidoglycan layer protecting the plasma membrane. ${ }^{175,178}$

A similar inhibition trend was observed for the use of AgNPs as active silver compound. The particles attach to the plasma membrane, change its permeability, disequilibrate the adenosine triphosphate pool and the proton motive force, and finally lead to cell death. ${ }^{152,161}$ However, there is also evidence that the adhesion of AgNPs to the cell wall does not necessarily lead to its disruption as, for instance, Choi et al. ${ }^{179}$ did not observe any plasma membrane leakage due to the presence of AgNPs. These different observations might be due to a variety of reasons such as different bacterial strains, AgNP concentration, size, shape, surface charge/functionalization (see below), or analytical methods.

Despite the tendency that the higher the concentration of AgNPs, the larger the inhibitory effect, regardless of the type of microorganism, ${ }^{152,166,180} \mathrm{Kim}$ et al. ${ }^{166}$ recently showed that Gram-positive $S$. aureus was less susceptible to the presence of AgNPs than Gram-negative E. coli. For further explanation, one should bear in mind that AgNPs are not necessarily neutral particles, as their surface can be positively or negatively charged due to the presence of stabilizing agents or additional surface modifications. ${ }^{166,181,182}$ This surface charge is important in terms of electrostatic interactions with the bacterial cell wall and is reflected by the zeta potential. One should expect that positively charged AgNPs are attracted by negatively charged lipopolysaccharides present in the outer membrane of Gramnegative bacteria, thus facilitating the interaction with the plasma membrane and enhancing the antimicrobial properties of AgNPs toward Gram-negative bacteria. ${ }^{183}$ In contrast, a thick rigid layer of peptidoglycan is, on the one hand, harder to penetrate and offers, on the other hand, only a limited number of anchoring sites for AgNPs, rendering their attack more difficult. ${ }^{183}$ The importance of the surface charge on the toxicity of AgNPs was confirmed by coating nanoparticles with citrate (citrate-AgNPs) and branched poly(ethyleneimine) (BPEIAgNPs). An antimicrobial assay was then carried out on Grampositive bacillus strains possessing a net negative charge on the membrane, ${ }^{181,184}$ which showed that there is a direct correlation between the surface charge and the antimicrobial activity of the nanoparticles. In this assay, the negatively charged citrate-AgNPs were much less toxic than the positively charged BPEI-AgNPs due to higher repulsive interactions between negatively charged NPs and the similarly charged membrane of the bacillus cells. ${ }^{181}$

Another possible mechanism for the integration or even penetration of AgNPs through the bacterial cell wall is the formation of breaks, so-called pits. The pit formation has recently been illustrated for Gram-negative ${ }^{165}$ and Grampositive ${ }^{185}$ bacteria using TEM. The proposed mechanism for the cell wall interaction and pit formation is depicted as proposed by Mirzajani et al. ${ }^{185}$ in Figure 13. It is suggested that

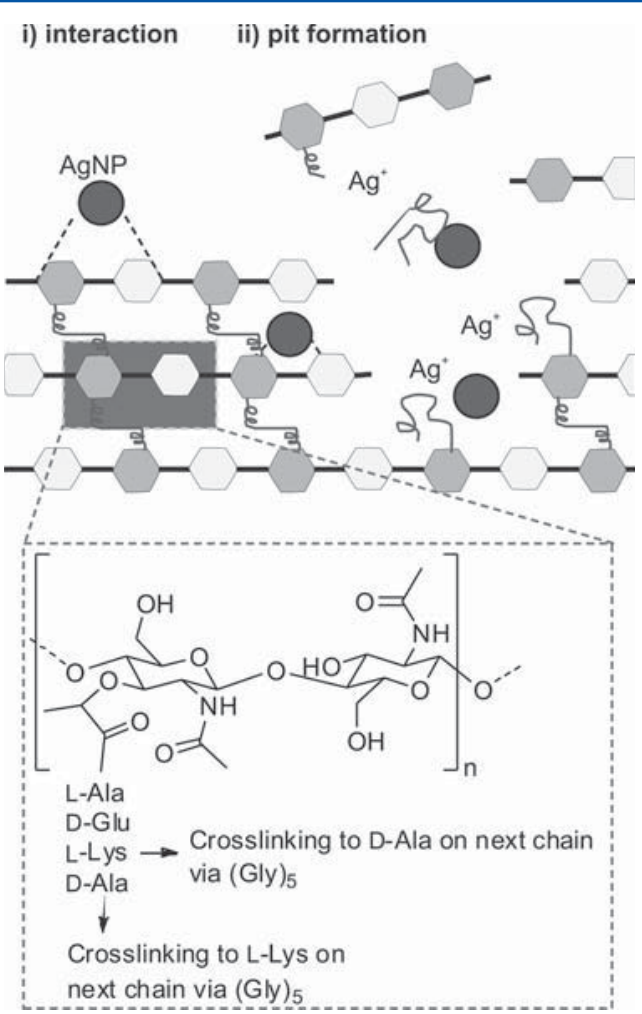

Figure 13. Depiction of the silver interaction with the bacterial cell wall leading to cell wall-damaging pit formation. Adapeted from ref 185. Copyright 2011 Institut Pasteur, published by Elsevier Masson SAS.

the AgNPs destroy the $\beta-1 \rightarrow 4$ glycosidic bonds that connect the building blocks of the peptidoglycan $N$-acetylglucosamine and $N$-acetylmuramic acid. This would finally lead to the release of these building blocks into the media. Indeed, the authors found an increased concentration of muramic acid in the media when AgNPs were present. ${ }^{185}$ Besides, it was shown by CD spectroscopy that the structure of the peptide branch was 
altered upon addition of AgNPs. Both findings underline the proposed mechanism experimentally.

Because both silver ions and AgNPs show a related behavior toward the cell wall, ${ }^{161}$ there is still an ongoing discussion on how much this antimicrobial activity of AgNPs can be ascribed to the release of $\operatorname{Ag}(\mathrm{I}) .^{166,186,187}$ It is even discussed in the literature that the release of $\mathrm{Ag}(\mathrm{I})$ is the only reason that has to be taken into account for the antimicrobial activity of AgNPs and that these particles can therefore utterly be considered as a silver ion reservoir ${ }^{155}$ or as modern Trojan horse ${ }^{188,189}$ that facilitates the release of silver ions inside the cell. There is experimental evidence for the release of ionic silver from AgNPs because the amount of free silver ions in solution can be measured. ${ }^{155,179}$ Several possible mechanisms for this $\mathrm{Ag}(\mathrm{I})$ release are discussed in the scientific literature. Choi et al. ${ }^{179}$ proposed an oxygen-mediated oxidative dissolution process according to eq 1 :

$$
4 \mathrm{Ag}+\mathrm{O}_{2}+2 \mathrm{H}_{2} \mathrm{O} \rightarrow 4 \mathrm{Ag}^{+}+4 \mathrm{OH}^{-}
$$

This theory is underlined by Liu and Hurt ${ }^{190}$ who indeed observed an increase of the $\mathrm{pH}$ upon AgNP dissolution. Further on, their thermodynamic analysis predicts citrateAgNPs to be unstable under aerobic biological conditions. Concerning the mechanism of silver ion release, they proposed a stepwise reduction of oxygen to water via peroxide intermediates rather than a four-electron transfer process. However, first results indicated that this oxidation process is slow, allowing the participation of other interaction pathways. ${ }^{190}$ Xiu et al. ${ }^{191}$ in this context postulate the formation of $\mathrm{Ag}_{2} \mathrm{O}-\mathrm{NPs}$ that are then able to release silver ions. Lok et al. ${ }^{155}$ indeed found experimental evidence that the formation of partially oxidized NPs is involved in their antimicrobial activity because only the $\mathrm{Ag}_{2} \mathrm{O}-\mathrm{NPs}$ exhibited antimicrobial activity against $E$. coli cells but not their precursor AgNPs synthesized by borohydride reduction of $\mathrm{AgNO}_{3}$.

Another release mechanism was hypothesized by MarambioJones and Hoek ${ }^{176}$ who suggested that the mechanism, proposed by Asharani et al. ${ }^{192}$ to take place in the mitochondria of eukaryotic cells (eq 2), could take place in bacterial cells as well.

$$
2 \mathrm{Ag}+\mathrm{H}_{2} \mathrm{O}_{2}+2 \mathrm{H}^{+} \rightarrow 2 \mathrm{Ag}^{+}+2 \mathrm{H}_{2} \mathrm{O}
$$

Liu et al. ${ }^{193}$ recently compared AgNPs to drug delivery systems, with the NP being the silver ion deliverer. They admitted however that a tuning of the release of the ionic silver is needed to improve current nanosilver technologies. Possible chemical approaches for a controlled release, in addition to the previously discussed primary release by oxidative dissolution, might be (i) inhibition by an insoluble $\mathrm{Ag}_{2} \mathrm{~S}$ film $\left(K_{\mathrm{sp}}=6 \times 10^{-51}\right.$ $\left.\mathrm{mol}^{3} \mathrm{~L}^{-3}\right)^{194}$ on the NP surface, (ii) surface passivation, (iii) preoxidation, (iv) polymeric coatings, or (v) the reversible surface binding of silver ions. ${ }^{193}$ Indeed, their first results showed that the inhibition of the bacterial growth is higher for preoxidized AgNPs than for untreated AgNPs, an observation that is in agreement with the above presented results of Lok et al., ${ }^{155}$ while AgNPs functionalized with 11-mercaptoundecanoid acid showed a much slower silver ion release as compared to the untreated samples. Recently, it was shown as well that AgNPs are transformed in wastewater treatment plants either completely into less toxic $\mathrm{Ag}_{2} \mathrm{~S}$-NPs or into core-shell particles consisting of a $\mathrm{Ag}$ core and a passivating $\mathrm{Ag}_{2} \mathrm{~S}$ shell. ${ }^{195}$ In general, it has been shown that the toxicity of both silver ions and AgNPs is influenced by the presence of silver coordinating counterions such as the appearance of common negatively charged ions, such as $\mathrm{Cl}^{-}, \mathrm{SO}_{4}{ }^{2-}, \mathrm{S}^{2-}$, and $\mathrm{PO}_{4}{ }^{3-}$. Their interaction with $\mathrm{Ag}(\mathrm{I})$ may cause its complexation and, depending on the concentration, also its precipitation, both leading to the further reduction of its bioavailability and toxicity (see also Figure 25, section 5.5). ${ }^{196-198}$ Recently, the correlation between the presence of common ligands versus antimicrobial activity of $\mathrm{AgNPs}$ and $\mathrm{Ag}(\mathrm{I})$ was investigated in vitro. ${ }^{191}$ It was found that the antimicrobial activity of AgNPs was less affected than the one of $\mathrm{Ag}(\mathrm{I})$ by the exposure to $\mathrm{Cl}^{-}$ under anaerobic conditions resulting in a drop in activity of $9 \%$ and $55 \%$, respectively. ${ }^{191}$

In summary, both silver ions and AgNPs are able to interact with the cell wall, which is one reason for their antimicrobial activity. However, concerning the toxicity of AgNPs, there is still some evidence needed to precisely answer the question on how much their antimicrobial activity depends on the release of ionic silver. It is widely accepted that the silver ion release is an important mechanism in terms of AgNP toxicity, but as section 3.1.3 will show, it does not seem to be the only mechanism involved.

3.1.2. Interactions with DNA, Enzymes, and Membrane Proteins. The interaction with the cell wall offers to the silver-containing species the possibility to penetrate the cell and to interact with compounds in its interior such as DNA or enzymes. Holt and Bard ${ }^{199}$ were able to determine the silver ion uptake by immobilized $E$. coli cells $\left(1 \mu \mathrm{M} \mathrm{AgNO}_{3}, 107.9\right.$ $\mu \mathrm{g} \mathrm{L}^{-1}$ ) resulting in a transport of $60 \%$ of the ionic silver inside the cell, while $40 \%$ remained bound to the outside of the cell without passing the plasma membrane. As was already mentioned in the previous subsection, silver ions caused the lysis of E. coli and S. aureus cells leading to a leakage of the cellular interior. ${ }^{175}$ Besides the detachment of the cell membrane from the cell wall, the TEM image showed an electron-light region in the center of the cell that was assigned to densely packed, so-called condensed DNA, which thereby loses its ability to replicate. ${ }^{175}$ This region is well separated from the electron-dense granules that contained a significant amount of silver and sulfur according to X-ray microanalysis, and it is surrounded by an electron-rich region. ${ }^{175}$ Thus, this is a clear indication for the interaction of silver ions with DNA. A similar observation was made for AgNPs when $\mathrm{Li}$ et al. ${ }^{162}$ concluded from TEM images the presence of condensed DNA in the cytoplasm of Gram-positive S. aureus cells treated with a commercially available AgNP solution ( $\mathrm{pH} 7$ ).

On a molecular level, Arakawa et al. ${ }^{200}$ identified guanine N7 and adenine $\mathrm{N} 7$ as the preferential binding site of $\mathrm{Ag}(\mathrm{I})$ in DNA by FTIR spectroscopy and capillary electrophoreses. $\mathrm{Ag}(\mathrm{I})$ has also been used for the construction of metalmediated base pairs. Recently, Johannsen et al. ${ }^{201}$ used artificial imidazole-Ag(I)-imidazole base pairs, as confirmed by NMR spectroscopy, for the construction of a DNA double-helix in which the metal ions are located along the helix axis without major conformational distortion of the helix. It is suggested that this artificial motive might be used in the future as recognition site. $^{201}$

Once inside the cell, silver compounds do not only interact with DNA, but with a number of enzymes as well. Li et al. ${ }^{162}$ found that AgNPs in contact with Gram-positive S. aureus inhibit the cell respiration and influence the expression of certain enzymes. It was shown that the enzymatic activity of the respiratory chain dehydrogenase decreased with increasing 
AgNP concentration. ${ }^{162}$ A similar proportional correlation between the AgNP concentration and the enzymatic activity of the respiratory chain dehydrogenase has been reported for Gram-negative E. coli cells treated with AgNPs. ${ }^{165}$ Also, for the mitochondrial respiratory chain such kind of interference has been observed. In this case, a reduced dehydrogenase activity was induced by the presence of AgNPs, which could be measured by the reduction of resazurin to resofurin (Figure 14). ${ }^{192}$ This reduction is performed only by vital cells leading to a color change from blue to pink (CellTiter Blue viability assay).

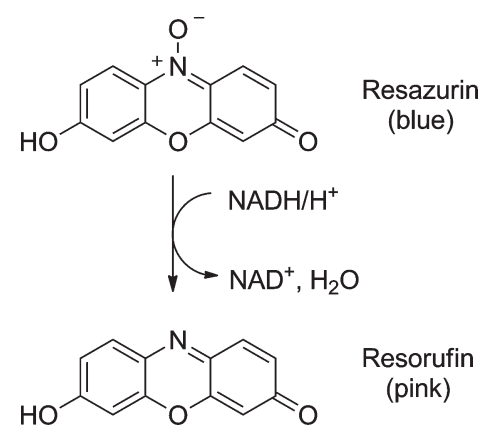

Figure 14. The reduction of resazurin to resofurin is only performed in the presence of vital cells and is responsible for the color change in the CellTiter Blue viability assay.

Silver ions can interfere with enzymes by binding to their amino acids forming silver-amino acid complexes (see section 2.1), which can also lead to the replacement of the native metal cation from its binding site in the enzyme. That $\mathrm{Ag}(\mathrm{I})$ is able to displace $\mathrm{Cu}(\mathrm{I})$ has, for example, been shown for ethylene receptors found in plants, where this metal ion exchange leads to the blocking of the ethylene perception. ${ }^{202}$ It has been reported that, for the interaction between silver ions and proteins, the silver binding to thiol groups, present, for instance, in the amino acid side chain of cysteine, is thought to be essential and leads to the inactivation of the enzyme. ${ }^{203-205}$ As was already discussed above, one should keep in mind that other amino acids should be much better ligands for silver ions based on their theoretically (Table 1) and experimentally determined silver ion affinities, and therefore this interaction might be overestimated if there are other amino acids with high silver ion affinity around such as arginine or histidine. However, the importance of the binding of silver toward cysteine has been shown, for example, by the destruction of an iron-sulfur cluster, a cofactor involved in many enzymatic reactions, due to the presence of $\mathrm{Ag}(\mathrm{I}) .^{206,207}$ For the enzyme fumarase $\mathrm{A}$, a member of the dehydratase family, it has recently been demonstrated in vitro using $E$. coli cells that the enzyme is inhibited due to the destruction of the cofactor. ${ }^{206}$ Besides, Kim et al. ${ }^{208}$ showed that silver ions are more effective antimicrobial agents against $E$. coli when the probably formed silver-cysteine complex is irradiated with ultraviolet-A light. Spectrophotometric and MALDI-TOF mass analyses revealed that the photochemical reaction of this silver-cysteine complex and the associated formation of monosulfide radicals are one of the key factors in the light-induced enhancement of the antimicrobial properties of silver ions. One of the most prominent examples for the interaction of silver ions with thiol groups in enzymes is the reduced nicotinamide-adenine dinucleotide (NADH) dehydrogenase. The direct effect of silver ions on the respiratory chain was first discovered 1974 by Bragg. ${ }^{209}$ A consequence of this interaction is the decoupling of the bacterial respiratory chain from the proton motive force across the plasma membrane, which results in the positive stimulation of the respiration before cell death. Holt and Bart ${ }^{199}$ were able to demonstrate this relation for E. coli. Presumably, the first experimental evidence for the silver-related collapse of the proton motive force has been given by Dibrov et al. ${ }^{210}$ and has been explained by the binding of $\mathrm{Ag}(\mathrm{I})$ to membrane proteins. It has been shown on the example of Vibrio cholera that the presence of silver ions at low (micromolar) concentrations is responsible for the leakage of protons through the cell membrane resulting in the collapse of the proton motive force.

The silver binding to $\mathrm{NADH}$ dehydrogenase also leads to the production of large quantities of ROS, which might be an explanation for the toxicity of silver ions to $E$. coli already at submicromolar concentrations. ${ }^{199}$ The participation of ROS in the toxicity of silver-containing compounds will be discussed in more detail in the following subsection.

3.1.3. Generation of Reactive Oxygen Species. The role of ROS in the antimicrobial properties of silver compounds has already been indicated above and has been depicted in Figure 12. The most important ROS discussed in this context are singlet oxygen, hydrogen peroxide, superoxide radical anion, and hydroxyl radical. ${ }^{211,212}$ They are known to target mainly lipids, DNA, RNA, and proteins, causing severe consequences such as the malfunction of membranes, proteins, and the DNA replication machinery. ${ }^{212}$ Because ROS are continuously produced from molecular oxygen as a byproduct during the metabolism of cells grown under aerobic conditions (eq 3), protective mechanisms were developed by these organisms. $^{212,213}$

$$
\mathrm{O}_{2} \stackrel{\mathrm{e}^{-}}{\rightarrow} \mathrm{O}_{2}^{-} \stackrel{\mathrm{e}^{-}+2 \mathrm{H}^{+}}{\longrightarrow} \mathrm{H}_{2} \mathrm{O}_{2} \stackrel{\mathrm{e}^{-}}{\rightarrow} \mathrm{OH}^{-}+\mathrm{OH}^{\cdot} \stackrel{\mathrm{e}^{-}+2 \mathrm{H}^{+}}{\longrightarrow} 2 \mathrm{H}_{2} \mathrm{O}
$$

This protection is reflected by the presence of two sensorregulator proteins called SoxR and OxyR, which in "unstressed" cells are latent. Once exposed to oxidative stress, which can be defined as the imbalance between the amount of ROS and the cell-own defense functions, the two aforementioned proteins become activated, hence sensing the presence of superoxide radical anion and hydrogen peroxide, respectively. ${ }^{211,212} \mathrm{~A}$ recent study on both Gram-negative and Gram-positive bacterial strains by Park et al. ${ }^{211}$ showed (i) that the antimicrobial activity of silver ions is higher under aerobic than under anaerobic conditions and (ii) that this enhancement is due to the formation of the superoxide radical anion, which, based on the amount of activation of SoxR and OxyR, is more important for the antimicrobial activity of silver ions than hydrogen peroxide. These findings underline a study by Holt and Bard ${ }^{199}$ who found that the formation of ROS is one consequence of the interaction of silver ions with enzymes in the respiratory chain (see section 3.1.2). The blocking of respiratory chain enzymes as a first step of an enhanced ROS production was also discussed by Gordon et al. ${ }^{207}$ who postulated the destruction of iron-sulfur clusters and subsequent Fenton reaction as origin of formed hydroxyl radicals. This hypothesis was among others supported by the upregulation of genes encoding oxidative stress response, hence supporting survival, for the investigated S. epidermidis, while other genes were downregulated resulting in a general growth arrest or an adaptive response to the presence of silver. 
Table 4. Transferable Silver Resistance Plasmids

\begin{tabular}{lclll} 
plasmid & molecular weight $(\mathrm{kb})$ & \multicolumn{1}{c}{ from } & \multicolumn{1}{c}{ to } & ref \\
pKK1 & 75 & P. stutzeri AG259 & P. putida CYM318 & high voltage electrotransformation \\
pUPI199 & 54 & Acinetobacter & E. coli K12 & conjugation \\
pMG101 & 180 & Salmonella & E. coli & conjugation \\
\hline
\end{tabular}

Another evidence for the participation of ROS in the mode of action of silver ions was obtained by testing the inhibitory properties of silver loaded zeolite (Ag-Z) toward the growth of E. coli. ${ }^{214}$ It was shown that (i) Ag-Z was active toward bacteria only in the presence of oxygen and (ii) the presence of ROS scavengers drastically curtailed the antibacterial activity of Ag-Z. Both observations suggest that ROS are formed and that they are one reason for the antimicrobial activity of the silver.

Kim et al. ${ }^{166}$ hypothesized in their work that the generation of ROS in relation with silver is not only based on the presence of $\mathrm{Ag}(\mathrm{I})$, be it through the addition of silver salts or their release from AgNPs, but can be traced back directly to AgNPs. Using EPR spectroscopy, they detected the release of free silver radicals from AgNPs. Although the damage of the cell membrane due to free radicals was proposed as an explanation for the observed phenomenon, no further experimental data were provided to underline this proposal. ${ }^{166}$ The dominant formation of the superoxide anion in vitro, mentioned above in the context of $\operatorname{Ag}(\mathrm{I})$, was also observed for E. coli cells treated with AgNPs by using stress specific bioluminescent bacteria, ${ }^{174}$ indicating that the form of the silver species is of minor importance. With regard to the different forms of silver, the same conclusion was drawn by Choi and $\mathrm{Hu}^{213}$ who observed ROS formation in vitro for all of the used silver formulations (AgNPs, ionic silver, and $\mathrm{AgCl}$ colloids). The possibility that the ROS generation is induced by the presence of light is also discussed, but only a poor correlation between the inhibitory activity and the photocatalytic ROS concentration was found. ${ }^{213}$

In contrast to the aforementioned studies, there are reports claiming that the formation of ROS has only a more or less negligible contribution to the antimicrobial properties of silver compounds. For instance, Sintubin et al. ${ }^{186}$ detected only a small amount of ROS in the presence of AgNPs by using a fluorescence assay based on $2^{\prime}, 7^{\prime}$-dichlorodihydrofluorescin diacetate, and the formation of an even minor extent of ROS was induced by ionic silver alone. In combination with their other results, they came to the conclusion that ROS production plays only a secondary role in terms of antimicrobial activity. The contribution of ROS formation is discussed controversially not only for AgNPs but also for $\mathrm{Ag}(\mathrm{I})$, as for example by Xiu et al., ${ }^{191}$ who reported that neither the presence nor the absence of oxygen influences the antibacterial activity of $\operatorname{Ag}(\mathrm{I})$. As a possible reason for the enhanced antimicrobial activity of AgNPs exposed to oxygen, they suggested the enhanced silver release rate of oxidized AgNPs, as was already mentioned in section 3.1.1. ${ }^{191}$ These sometimes contradictory results on the importance of ROS in terms of silver's antimicrobial properties clearly lead to the bottom line that there is still a lot of effort to be made to understand the mode of action.

To conclude this subsection on the antimicrobial properties of silver compounds, there is no doubt that silver acts in a multidirectional fashion. This complexity renders its study and understanding rather difficult because there are several factors involved in the puzzle such as: (i) different interaction pathways with the bacterial cell, (ii) different bacterial strains, ${ }^{152,166}$ (iii) different forms of silver present in and outside the cell (ionic silver versus AgNPs), and (iv) the concentration of the silver compound ${ }^{180,215}$ as well as the one of silver coordinating ligands. ${ }^{191}$ When working with AgNPs, several additional factors become important such as their size, ${ }^{157,158,213}$ the presence of ligand coatings ${ }^{193}$ influencing among others their surface charge, ${ }^{181}$ and finally even their shape. ${ }^{180}$

Another rising issue are reports on resistant bacteria even though one advantage of silver as antimicrobial compound is claimed to be its broad mode of action, which should make the resistance building rather difficult. ${ }^{216-218}$ On the one hand, it poses a threat and danger to the medical field, ${ }^{219,220}$ but on the other hand, it constitutes a potential for the biosynthesis of AgNPs from an industrial point of view. The bacterial resistance to the presence of silver is discussed in section 3.2, whereas methods for the biosynthesis of AgNPs are described in section 3.3.

\subsection{Bacterial Resistance Mechanisms against Ag(I)}

The use of silver as an antimicrobial agent was abandoned in the middle of the 20th century in response to the discovery and development of antibiotics, ${ }^{21}$ but with the fast increase of resistance build-up in bacteria, especially against conventional, narrow-spectrum antibiotics, silver-based compounds enjoy again great popularity. ${ }^{180}$ As was briefly mentioned above, the biggest advantage of silver as an antimicrobial agent originates from its multidirectional activity. Thus, resistance development is very difficult and requires plenty of mutations in the cell. ${ }^{180}$ However, it was discovered that some bacterial strains, regularly exposed to relatively high (millimolar) concentrations of silver, were able to grow in this silver-rich environment. ${ }^{222-228}$ This surprising discovery led to an intensified interest in the silver resistance machinery. Bacterial resistance to toxic metals is in general encoded on plasmids, but in rare cases also on the bacterial chromosomes. ${ }^{229,230}$ In the case of silver resistance, the most frequently discussed plasmids in the scientific

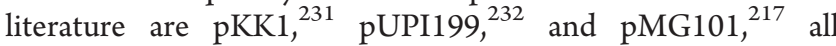
shown in Table 4.

The molecular mechanisms behind the silver resistance are still not completely understood and currently rely on two leading theories: The first one is the accumulation and storage of silver, ${ }^{94}$ and the second one is a silver efflux mechanism. ${ }^{221}$ Both theories are based on the same concept, which is the detoxification of the cell. ${ }^{233}$ The former, often controversially discussed in the literature, ${ }^{10,234}$ predicts the accumulation of ionic silver that is in turn transformed to less harmful $\operatorname{Ag}(0),{ }^{94,235}$ whereas the latter anticipates that silver ions are transported out of the cell. ${ }^{10}$

3.2.1. Accumulation and Storage-Based Mechanism. Having a closer look at the first proposed mechanism, one of the earliest investigations on the accumulation of $\mathrm{Ag}(\mathrm{I})$ is based on the silver resistant $P$. stutzeri strain AG259. This strain naturally found in the soil of silver mines was for the first time analyzed by Haefeli et al. ${ }^{236}$ in 1984 . The presence of the pKK1 plasmid was exclusively observed in Ag-resistant colonies. Slawson et al. ${ }^{154,237}$ revealed that the Ag-resistant (AG259) and 


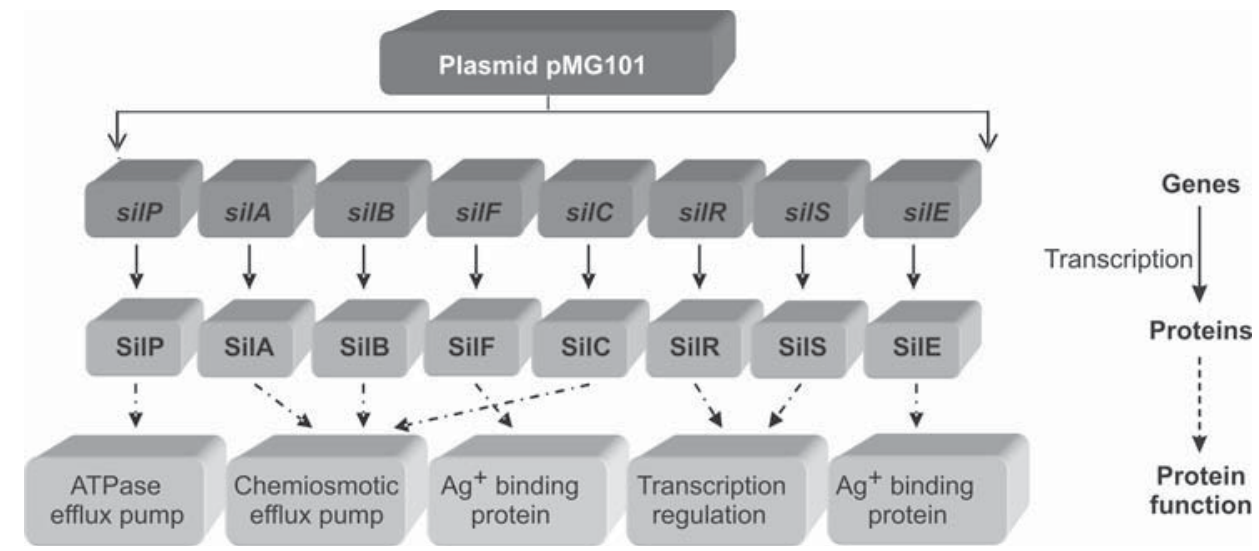

Figure 15. Origin of silver resistance in Salmonella. Adapted with permission from ref 10. Copyright 2006 John Wiley and Sons. Adapted with permission from ref 11. Copyright 2006 Springer Science and Business Media.

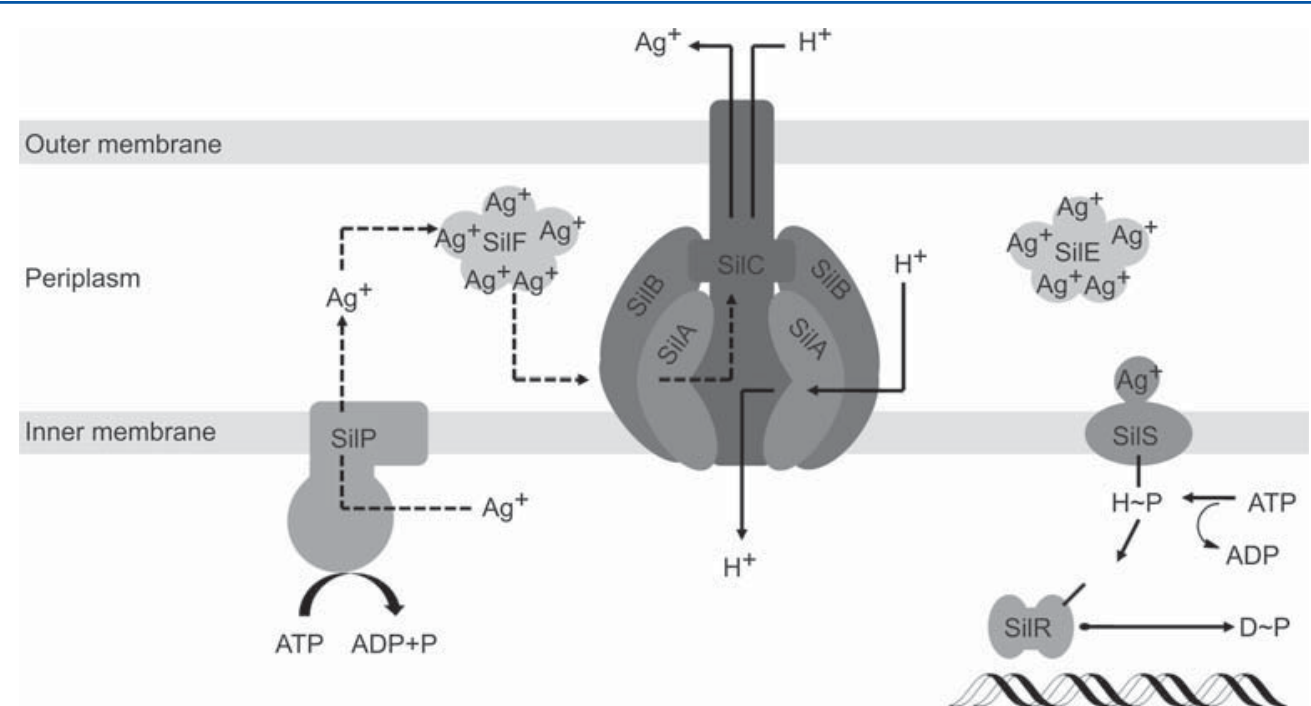

Figure 16. Protein products of bacterial plasmid silver resistance genes. Adapted with permission from ref 10. Copyright 2006 John Wiley and Sons. Adapted with permission from ref 11. Copyright 2006 Springer Science and Business Media.

the Ag-sensitive (JM303) P. stutzeri strains were both able to accumulate silver, but only the resistant strain was able to form dense metal deposits as was observed using TEM and energy dispersive X-ray analysis. Because high sulfur content was found for the resistant strain AG259, the production of hydrogen sulfide was suggested to play a role in the formation of metal deposits that were claimed to be silver sulfide. Later, Klaus et al. ${ }^{94}$ reported the formation of nanosized crystals of diverse types (elemental silver, silver sulfide, undetermined composition) and shapes using the same silver-resistant strain, indicating that already small changes in the experimental conditions might have a huge effect on the outcome of the experiment.

As shown in Table 4, the plasmid pUIP199 has also been discussed to be responsible for the accumulation of silver ions, but experimental data using this plasmid are rare. It was shown that the plasmid and, with it, the silver resistance were transferable from Acinetobacter baumannii to E. coli and that it did not encode for resistances against other metals or antibiotics. However, it was not clear whether the silver was accumulated on the surface of the cell or in its interior. ${ }^{232}$

3.2.2. Efflux Pump-Based Mechanism. The second explanation for silver resistance, related to the third plasmid pMG101, is based on an efflux system, which was for the first time postulated by Silver et al. ${ }^{238}$ in 1999. Since then, this is the most deeply studied and most frequently cited model for silver resistance. The research on Salmonella isolated from a hospital burn ward revealed that the silver resistance is encoded by sil genes located on the transferable plasmid pMG101 (Figure 15). ${ }^{217}$ This plasmid is also responsible for further resistances against other heavy metals or antibiotics. ${ }^{217,223}$ The encoded resistance system shown in Figure 16 consists of two $\mathrm{Ag}(\mathrm{I})$ binding proteins (SilE and SilF) and two different efflux pumps, which are a P-type ATPase (SilP) and a three-protein chemiosmotic cation/proton antiporter (SilCBA). The production of these sil-proteins only takes place if the cells are grown in the presence of silver, and it is regulated by a system consisting of an ATP kinase (SilS) as a membrane sensor and a transcriptional regulatory protein (SilR) as responder. First, extracellular signals are detected by SilS, and subsequently an aspartate residue on SilR is trans-phosphorylated leading to the synthesis of other sil-proteins. ${ }^{10}$ Because pumping systems as formed by SilCBA ${ }^{10,11}$ are well investigated due to their presence in resistance machineries against other heavy metals as well, ${ }^{230,239}$ we will concentrate in this Review on the role of the two Ag-binding proteins.

Although SilE and SilF are both expected to bind silver, they differ significantly in their composition and their mode of 
Table 5. Amino Acid Sequence of CusF and $\operatorname{SilF}^{247,248} a$

\begin{tabular}{|c|c|c|c|c|}
\hline \multirow{2}{*}{$\begin{array}{c}\text { protein } \\
\text { CusF }\end{array}$} & \multicolumn{4}{|c|}{ amino acid sequence } \\
\hline & 10 & 20 & 30 & 40 \\
\hline & MKKALQVAMF & SLFTVIGFNA & QANEHHHETM & SEAQPQVISA \\
\hline & 50 & 60 & 70 & 80 \\
\hline & TGVVKGVDLE & SKKITIHHDDP & IAAVNWPEMT & MRFTITPQTK \\
\hline & 90 & 100 & 110 & \\
\hline & MSEIKTGDKV & AFNFVQQGNL & SLLQDIKVSQ & \\
\hline \multirow[t]{6}{*}{ SilF } & 10 & 20 & 30 & 40 \\
\hline & MLKHISHGDM & NAASDASVQQ & VIKGTGIVKD & IDMNSKKITI \\
\hline & 50 & 60 & 70 & 80 \\
\hline & SHEAIPAVGW & PAMTMRFTFV & NADDAINALK & TGNHVDFSFI \\
\hline & 90 & INVTQS & & \\
\hline & QQGNISLLKS & & & \\
\hline
\end{tabular}

${ }^{a_{a}}$ The amino acids responsible for Ag-binding (H-histidine, M-methionine, W-tryptophan) are underlined.

action. ${ }^{11}$ SilE is a small periplasmic protein consisting of 143 amino acids, which is $47 \%$ identical to the metal binding peptide PcoE from the plasmid copper resistance system of $E$. coli. ${ }^{217}$ In both proteins (SilE and PcoE), 10 histidine residues are located on identical positions. ${ }^{217}$ SilE possesses a high specificity for $\mathrm{Ag}(\mathrm{I})$ over $\mathrm{Cu}(\mathrm{II})$ and $\mathrm{Cd}(\mathrm{II}),{ }^{217}$ and the 10 histidine imidazole nitrogen atoms were determined by NMR spectroscopy as the primary silver binding sites. ${ }^{10}$ This binding is supposed to lead to a structural change from a random coil to a predominantly $\alpha$-helical system. ${ }^{10}$ However, to the best of our knowledge, the publication given as reference for the structural changes upon $\mathrm{Ag}(\mathrm{I})$ binding never appeared, so that experimental proof is missing.

SilE, silP, and silS gene homologues were also found in a silver-resistant Morganella strain carrying out an extracellular biosynthesis of AgNPs (see section 3.3). ${ }^{216}$ The nucleotide sequence of this silE homologue showed $99 \%$ similarity with the silE found in Salmonella. Recently, the screening of 10 Morganella strains used for the production of AgNPs revealed the presence of a silE gene homologue, thus emphasizing the role of silver resistance genes in the production of nanoparticles. ${ }^{240}$ Contrary to earlier findings that the silE gene is always associated with the presence of other sil genes, ${ }^{10}$ it was present alone in two methicillin-resistant S. aureus (MRSA) and an isolate of methicillin-resistant coagulase-negative staphylococci strain. ${ }^{220}$ These bacteria were not able to survive in the presence of silver ions for a long period of time, indicating that the sile gene alone does not provide sufficient protection. $^{220}$

The second silver binding protein encoded in the sil system, $\mathrm{SilF}$, is a homologue of the chromosomal encoded ${ }^{241}$ CusF in E. coli (50\% identity). ${ }^{11}$ CusF is part of the recently reviewed ${ }^{242}$ Cus efflux system that removes $\mathrm{Cu}(\mathrm{I})$ and $\mathrm{Ag}(\mathrm{I})$ via a methionine shuttle. The small periplasmic protein CusF (110 amino acids), whose crystal structure is known with ${ }^{243}$ and without $^{244} \mathrm{Ag}(\mathrm{I})$, is proposed to serve as a metallochaperone. ${ }^{245}$ Contrary to other known metal carrier proteins, CusF acts in form of a monomer in which three amino acids (Met47, Met49, His36) are involved in the full coordination of $\mathrm{Ag}(\mathrm{I})$, and Trp44 is capping the metal binding site of the molecule potentially by a $\eta^{2}-\pi$ interaction. ${ }^{243,246}$ CusF has a higher binding affinity for $\mathrm{Ag}(\mathrm{I})$ than for $\mathrm{Cu}(\mathrm{I})$, which may be ascribed, for instance, to the different affinities of those ions to sulfur ligands or to their different sizes. According to X-ray absorption spectroscopy, the environment for $\mathrm{Cu}(\mathrm{I})$ is similar to the one found for $\operatorname{Ag}(\mathrm{I})$, thus suggesting a similar binding situation. ${ }^{245}$ The four amino acids forming the principal binding motif are preserved in the sequence of the 96 amino acid long SilF as shown in Table 5. ${ }^{247,248}$ Consequently, SilF is predicted to adopt a $\beta$-sheet structure similar to CusF in which a single histidine and two methionine residues bind the silver cation. ${ }^{11}$ In the current models, both metal binding proteins CusF and SilF are responsible for the capture and subsequent transport of $\mathrm{Ag}(\mathrm{I})$ to the corresponding efflux pumps, where the toxic metal ion is finally eliminated from the cell.

At a first glance, both models explaining the silver resistance in bacteria seem to be contradictory to each other because the first one involves the accumulation of the metal ion inside the cell while the second one eliminates specifically this metal ion. However, their authenticity might be exemplified by the bioproduction of AgNPs (discussed in detail in the following section), where the bioaccumulation of $\mathrm{Ag}(\mathrm{I})$ leads to intracellular synthesis, ${ }^{94}$ and the so-formed particles are extracted by an efflux system. ${ }^{249}$ For a complete understanding of the resistance machinery, a lot of open questions still remain to be answered such as (i) whether there is a maximum $\operatorname{Ag}(\mathrm{I})$ concentration manageable by the efflux pumps and the storage system, (ii) whether the formation of AgNPs can really protect cells considering that they are doubtlessly able to release $\mathrm{Ag}(\mathrm{I})$, and (iii) how the resistance transfer or the occurrence of cross resistances can be prevented. In addition, it is not clear from the literature whether the same transporters are involved in the metabolism of AgNPs.

\subsection{Synthesis of Silver Nanoparticles by Means of Bacteria}

Using the possibilities offered by silver-resistant bacteria, there has recently been a growing interest in the bioproduction of AgNPs. The main advantages of using bacteria over traditional chemical methods are that they generate less waste, prevent the generation of harmful byproducts, and require less energy in the production process. Because this manufacturing is considered as ecologically friendly, it is often termed "green synthesis". ${ }^{158,216,250}$ In addition, (i) bacteria are relatively easy to handle, (ii) the recovery and purification (downstream processing) of the formed AgNPs is simple, and (iii) their genetic manipulation is straightforward allowing the tuning of the formed NPs. ${ }^{216}$ Besides, modifications of the experimental conditions such as the $\mathrm{Ag}(\mathrm{I})$ concentration, ${ }^{251,252}$ the bacterial strain, ${ }^{253} \mathrm{pH}$ of the media, ${ }^{94,252,253}$ reaction time, ${ }^{94}$ or reaction temperature ${ }^{249,251,252}$ result in AgNPs differing, for instance, in size, ${ }^{94,252,253}$ amount, ${ }^{251,253}$ shape, $^{249}$ or morphology. ${ }^{94}$ 
Nevertheless, several challenges are posed to the biosynthesis of NPs such as (i) the toxicity of reactants and products for the microorganisms themselves, (ii) the high production costs due to sterile conditions, costly culture media, or large reaction volumes, and (iii) the low productivity. ${ }^{251}$ However, the use of bacteria living under extreme conditions might solve some of these issues and has indeed already led to promising results. For instance, the use of the thermophilic bacterium Ureibacillus thermosphaericus ( $U$. thermosphaericus) allowed cost savings because, due to the possibility of a higher reaction temperature $\left(60-80{ }^{\circ} \mathrm{C}\right)$, sterile conditions were dispensable. ${ }^{251}$ A second example is the use of Lactobacillus fermentum (L. fermentum) possessing a high $\mathrm{pH}$ tolerance that allowed the AgNP synthesis under basic conditions $(\mathrm{pH}$ 11.5) resulting in enhanced silver recovery and reduction rates. ${ }^{253}$

With bacteria as biocatalysts, one differentiates between intracellular $^{254}$ and extracellular ${ }^{216}$ AgNP production. If particles are generated and accumulated in various locations inside the cell, it is defined as intracellular synthesis. ${ }^{254}$ For the extracellular NP production, either the cells first take up silver cations, reduce them in their interior, and then excrete the particles to the outside of the cell, ${ }^{216}$ or only the cell-free supernatant is used to produce AgNPs, which contains secondary metabolites produced by the cells but no cells themselves. $^{163}$

In both cases, intra- or extracellular, the bioreduction of silver can be carried out enzymatically and nonenzymatically. The enzymatic reduction of $\operatorname{Ag}(\mathrm{I})$ to $\operatorname{Ag}(0)$ occurs for instance in Gram-positive Bacillus licheniformis (B. licheniformis) via a $\mathrm{NADH}$-dependent nitrate reductase. This enzyme is located on the membrane and is known to biosynthesize other nanoparticles as well such as gold or iron oxide. ${ }^{255}$ The enzyme participation was investigated by the addition of several enzyme inhibitors as, for instance, sodium azide, which reduced the NP formation by $90 \%$, while others showed merely a partial inhibition. ${ }^{256}$ A partial inhibition of the AgNP formation was also observed for strains of Gram-negative Enterobacteriaceae treated with the natural monoterpene ketone piperitone (6isopropyl-3-methyl-1-cyclohex-2-enone). ${ }^{257}$ The participation of enzymes has also been shown by Law et al. ${ }^{258}$ for the extracellular silver reduction performed by Geobacter sulfurreducens (G. sulfurreducens). Mutation experiments suggest a key role of $C$-type cytochromes in the electron transfer chain trough the periplasm and the outer membrane resulting in the subsequent extracellular reduction of ionic silver. However, to the best of the authors' knowledge, no experiments concerning the fate of the involved proteins of G. sulfurreducens have been conducted to observe possible changes in the structure, shape, or chemistry of the oxidized proteins. A second possible mechanism for the extracellular reduction of metal ions performed by G. sulfurreducens is the involvement of cytochrome-free bacterial pili functioning as nanowires that can be described as conductive protein appendage formed through the polymerization of a single helical peptide subunit, the so-called pilin. These pili are used to transport the electrons that are necessary for the reduction of the metal. ${ }^{259}$ However, the mechanism of the electron transport through these pili is until now not well understood, but there is evidence that the $\alpha$ helical conformation of the pilin is of importance. ${ }^{260}$

For bacteria from the genus Morganella, both enzymatic and nonenzymatic production of AgNPs has been discussed. While Parikh et al. ${ }^{216}$ proposed the release of the silver binding protein SilE (see previous section) to the outside of the cell that then enables the formation of AgNPs by using the cell-free supernatant, a recent study by Ramanathan et al. ${ }^{249}$ based on electrochemical experiments suggested the involvement of a silver reductase enzyme. After this intercellular reduction, the formed AgNPs are subsequently released out of the cells. ${ }^{249}$ The fact that all Morganella strains being able to synthesize AgNPs contained silE gene homologues underlines the assumption that the AgNPs formation is associated with the silver-resistance machinery. ${ }^{216,240,249}$ In addition, the study of Ramanathan et al. ${ }^{249}$ showed for the first time that the shape of the formed AgNPs is a function of the temperature during the growth of Morganella psychrotolerans (M. psychrotolerans) used as biocatalyst, even though microorganisms predominantly tend to produce spherical nanoparticles, which are the easiest to create in terms of energy consumption and thus minimize the effort of the cells (Table 6). ${ }^{249}$

The advantages of a nonenzymatic bioreduction process are that no special conditions to preserve the enzyme activity are needed and that high concentrations of $\mathrm{Ag}(\mathrm{I})$ can be used. ${ }^{277}$ In general, the nonenzymatic reduction takes place via a twostep mechanism in which the biosorption of silver cations is followed by their bioreduction. The first step occurs via the binding of $\mathrm{Ag}(\mathrm{I})$ by biomolecules present on the surface of the bacterial cell wall such as carbohydrates (via their hydroxyl groups) 253,284 $^{\text {or amino acids }}{ }^{284}$ (via their side chains as discussed in detail in section 2). The silver reduction step is ascribed to the presence of reducing groups in the bacterial cell wall. One example are polysaccharides present in the cell wall of Gram-positive bacteria, which are able to carry out the nonenzymatic reduction of silver, whereas Gram-negative bacteria lacking those sugar polymers are not. ${ }^{253}$ In case of Gram-positive lactic bacteria, the presence of exopolysaccharides does not only provide additional silver binding sites, but they also serve as electron donors leading to silver reduction. Along with increasing $\mathrm{pH}$, an augmented silver reduction rate was observed, which can be explained by an enhanced formation of the reactive aldehyde present in the open-chain sugar. ${ }^{253}$ The proposed mechanism is shown in Figure 17.

A nonenzymatic silver reduction was also reported in the biomass of Gram-negative Aeromonas or Gram-positive Corynebacterium. ${ }^{277,285}$ In both cases, the accumulation of $\mathrm{Ag}(\mathrm{I})$ and $\left[\mathrm{Ag}\left(\mathrm{NH}_{3}\right)_{2}\right]^{+}$on the cell wall is proposed, which are then subsequently reduced by aldehydes or ketones to metallic silver. In addition to the above-mentioned nonenzymatic factors contributing to the synthesis of AgNPs, it has also been reported that hydroquinones behave as excellent electron shuttles, which are released into the medium by Enterobacteriaceae, and may be responsible for the extracellular silver reduction that was found by using the cell-free supernatants. ${ }^{257}$

With increasing interest in the green production of nanoparticles, a growing number of organisms or their extracts are tested for their silver-reducing properties. Besides bacteria, ${ }^{272-275,280}$ also several fungi ${ }^{261-271}$ and plants ${ }^{108,281-283}$ exhibit a potential for this green synthesis (Table 6).

In summary, there are several possibilities for the formation of AgNPs using bacterial cells, the intracellular or extracellular synthesis that can both occur either enzymatically or nonenzymatically. The complexity of the processes inside and outside of the cells makes a deep understanding of the mechanisms behind the AgNP formation rather difficult. In the majority of studies, the action of sil proteins is suspected to be a crucial factor in the formation of AgNPs. ${ }^{249}$ Nevertheless, little 
Table 6. Synthesis of AgNPs by Different Microorganisms ${ }^{a}$

\begin{tabular}{|c|c|c|c|c|}
\hline size $[\mathrm{nm}]$ & shape & organism & strain & ref \\
\hline $5-25$ & spherical & F & Aspergillus fumigatus & 261 \\
\hline $7-50$ & spherical & $\mathrm{F}$ & Aspergillus fumigatus & 262 \\
\hline $10-100$ & spherical & $\mathrm{F}$ & $\begin{array}{l}\text { Cladosporium } \\
\text { cladosporioides }\end{array}$ & 263 \\
\hline $20-50$ & spherical & $\mathrm{F}$ & Fusarium oxysporum & 264 \\
\hline $2-5$ & spherical & $\mathrm{F}$ & MKY3 & 265 \\
\hline $3-8$ & hexagonal & & & \\
\hline$\sim 15$ & spherical & $\mathrm{F}$ & mushroom extract & 266 \\
\hline \multirow[t]{2}{*}{$5-25$} & spherical & $\mathrm{F}$ & Penicillium & 267 \\
\hline & & & Fellutanum & \\
\hline $23-105$ & n.s. & $\mathrm{F}$ & $\begin{array}{l}\text { Penicillium } \\
\text { brevicompactum WA } \\
2315\end{array}$ & 268 \\
\hline $50-200$ & pyramidal & $\mathrm{F}$ & $\begin{array}{l}\text { Phaenerochaete } \\
\text { chrysosporium }\end{array}$ & 269 \\
\hline$\sim 71$ & spherical & F & Phoma sp. 3.2883 & 270 \\
\hline n.s. & n.s. & $\mathrm{F}$ & Phytophora infestans & 271 \\
\hline $4-5$ & spherical & $\mathrm{G}(+)$ & B. cereus & 272 \\
\hline $40-50$ & n.s. & $\mathrm{G}(+)$ & B. licheniformis & 273 \\
\hline$\sim 50$ & n.s. & $\mathrm{G}(+)$ & B. licheniformis & 255 \\
\hline $10-80$ & n.s. & $\mathrm{G}(+)$ & B. licheniformis & 256 \\
\hline $5-15$ & seed-like & $\mathrm{G}(+)$ & Bacillus sp. & 274 \\
\hline $10-50$ & spherical & $\mathrm{G}(+)$ & Brevibacterium casei & 275 \\
\hline $10-15$ & n.s. & $\mathrm{G}(+)$ & Corynebacterium sp. SH09 & 276 \\
\hline $11-20$ & spherical & $\mathrm{G}(+)$ & L. fermentum & 253 \\
\hline n.s. & n.s. & $\mathrm{G}(+)$ & Pediococcus pentosaceus & \\
\hline n.s. & n.s. & $\mathrm{G}(+)$ & Enterococcus faecium & \\
\hline n.s. & n.s. & $\mathrm{G}(+)$ & Lactococcus garvieae & \\
\hline $15-500$ & n.s. & $\mathrm{G}(+)$ & Lactobacillus & 254 \\
\hline $20-30$ & spherical & $\mathrm{G}(+)$ & Streptomyces hygroscopicus & 163 \\
\hline$<20$ & n.s. & $\mathrm{G}(-)$ & Aeromonas sp. SH10 & $\begin{array}{l}277 \\
278\end{array}$ \\
\hline$\sim 50$ & spherical & $\mathrm{G}(-)$ & E. coli & 252 \\
\hline $5-20$ & spherical & $\mathrm{G}(-)$ & E. coli & 164 \\
\hline $5-35$ & spherical & $\mathrm{F}$ & Aspergillus niger & \\
\hline $28-122$ & spherical & $\mathrm{G}(-)$ & Enterobacteriaceae & 257 \\
\hline$\sim 30$ & spherical & $\mathrm{G}(-)$ & G. sulfurreducens & 258 \\
\hline $2-5\left(20^{\circ} \mathrm{C}\right)$ & spherical & $\mathrm{G}(-)$ & M. psychrotolerans & 249 \\
\hline $\begin{array}{l}70-100 \\
\left(4{ }^{\circ} \mathrm{C}\right)\end{array}$ & nanoplates & & & \\
\hline $100-150$ & triangular & & & \\
\hline $100-150$ & hexagonal & & & \\
\hline $15-25$ & spherical & $\mathrm{G}(-)$ & Morganella sp. & 216 \\
\hline $10-50$ & $\begin{array}{l}\text { quasi- } \\
\text { spherical }\end{array}$ & $\mathrm{G}(-)$ & Morganella spp. & 240 \\
\hline$<200$ & $\begin{array}{l}\text { triangular } \\
\text { hexagonal } \\
\text { spherical }\end{array}$ & $\mathrm{G}(-)$ & P. stutzeri AG259 & $\begin{array}{l}94, \\
279\end{array}$ \\
\hline$\sim 40$ & spherical & $\mathrm{G}(-)$ & Plectonema boryanum & 280 \\
\hline $1-200$ & octahedral & & & \\
\hline n.s. & anhedral & & & \\
\hline $10-100$ & spherical & $\mathrm{G}(-)$ & U. thermosphaericus & 251 \\
\hline $2-3$ & icosahedral & $\mathrm{P}$ & Alfaalfa sprouts & 281 \\
\hline $10-20$ & spherical & $\mathrm{P}$ & $\begin{array}{l}\text { Azadirachta indica } \\
\text { (Neem) }\end{array}$ & 282 \\
\hline $55-80$ & $\begin{array}{l}\text { quasi- } \\
\text { spherical }\end{array}$ & $\mathrm{P}$ & Cinnamomum camphora & 283 \\
\hline
\end{tabular}

${ }^{a} \mathrm{~F}$, fungi; $\mathrm{G}(+)$, Gram positive bacteria; $\mathrm{G}(-)$, Gram negative bacteria; P, plants; n.s., not specified; sp., single species; spp., several species.

progress in this area has been made because the mechanistic studies in this area are limited predominantly to the detection
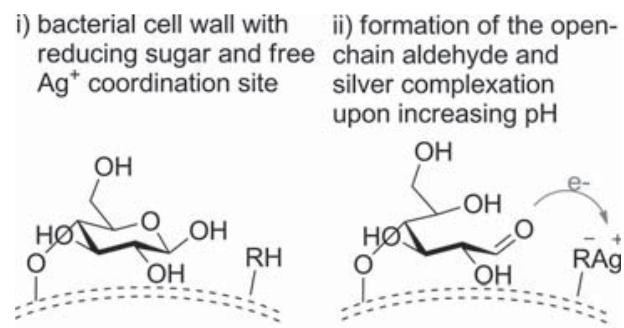
iii) silver reduction has taken place and the aldehyde is oxidized to the coresponding carboxilic acid

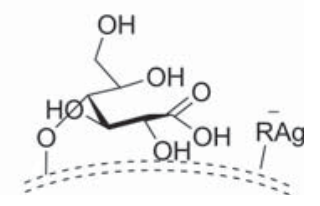

Figure 17. Possible steps of the silver reduction by sugars present on the cell surface in combination with free silver coordination sites (R). Adapted with permission from ref 253. Copyright 2006 Springer Science and Business Media.

of silE homologues. ${ }^{216,240,249}$ Even though a deep understanding especially on the molecular level is still missing, there are promising results showing that the tuning of AgNPs in size and shape is possible with bacteria as biocatalysts. Nonetheless, the authors of this Review are convinced that a detailed understanding of the involved processes is a prerequisite for the controlled and directed synthesis of AgNPs as well as for their safe application as will be discussed for the medical field in the two following sections.

\section{SILVER-BASED BIOMATERIALS IN THE MEDICAL FIELD}

Implant-related infections still remain an issue in medicine. For example, $4.3 \%$ of orthopedic and $7.4 \%$ of cardiovascular implants inserted in humans annually in the U.S. become infected. ${ }^{286}$ Implant infections often result in pain for the patients and malfunction of the implant, which may lead to the replacement of the implant, or in extreme cases amputation or death of the patient. ${ }^{287,288}$ Infections also considerably increase medical costs. In the U.S. alone, costs of more than $\$ 3$ billion are generated every year due to such infections. ${ }^{286}$ Because the number of patients receiving an implant is continuously increasing and bacteria are becoming more and more resistant to antibiotics, ${ }^{289}$ new strategies to prevent and cure implantrelated infections are currently under investigation and development. ${ }^{290}$ For an implant to function properly, it should be well integrated by the host cells. When bacteria are present, they compete with the host cells for the adhesion on the implant surface. When a biofilm ${ }^{291}$ is formed on the implant surface, that is, when there is an aggregation of bacteria adhering to each other on the implant surface, the implant cannot be well integrated by the host cells. This often leads to a malfunction of the implant or to other health complications. Moreover, it is normally impossible to eradicate this kind of infections in vivo so that the only solution is the complete removal of the implant. ${ }^{146}$ The ideal antimicrobial implant would therefore prevent any bacterial adhesion without affecting host cell integration. Silver is a good candidate because it has a high toxicity toward bacteria (see section 3 ), but a lower toxicity toward eukaryotes (see section 5) offering a "therapeutic window" for the use of silver-based biomaterials. ${ }^{56}$ The EPA published a reference dose (RFD) for oral silver exposure of $5 \mu \mathrm{g} \mathrm{kg}^{-1} \mathrm{~d}^{-1}$ with a critical dose estimated at 
$14 \mu \mathrm{g} \mathrm{kg}^{-1} \mathrm{~d}^{-1} .{ }^{12}$ Interestingly, Bunyan et al. showed that rats supplemented with selenium or vitamin $\mathrm{E}$ tolerated a silver exposure of as high as $140 \mu \mathrm{g} \mathrm{kg}^{-1} \mathrm{~d}^{-1}$. Thus, a $70 \mathrm{~kg}$ adult should not exceed $350 \mu \mathrm{g}$ per day. ${ }^{292}$

As it will be discussed in section 5, silver-containing biomaterials are already present on the market, ${ }^{293}$ for example, in wound dressings, ${ }^{88,294,295}$ contraceptive devices, ${ }^{296}$ endotracheal tubes, ${ }^{297}$ and bone prostheses. ${ }^{293}$ They are also intensively studied for preventing and treating infections on burns, ${ }^{298}$ prostheses, ${ }^{299}$ catheters, ${ }^{300,301}$ vascular grafts, ${ }^{119}$ surgical instruments, ${ }^{128}$ and dental devices. ${ }^{47,90}$ One advantage of using silver-containing materials to coat biomaterials, in addition to silver's good biocompatibility and good antimicrobial activity, is that they often protect both the inner and outer surfaces and the proximity of a medical device in such a way that the coating does not always need to cover the whole implant surface to protect the implant from infection. ${ }^{31,38}$

The use of silver in medicine is highly debated, and there are some contradictory studies as will be seen in the following. Many factors influence the experimental observations during antimicrobial activity and biocompatibility experiments. Among others, the choice of the experiment type for the evaluation of antimicrobial efficiency has a major importance on the experimental conclusions, as it was pointed out by Klueh et al. ${ }^{302}$ They observed that batch colonization experiments have many disadvantages as compared to flow cell experiments. In batch assays, it is difficult to define the hydrodynamic conditions, to measure the correct cell deposition rate, to determine the cell surface concentration, and to control the reactor conditions, which are constantly changing. On the other hand, flow cell systems mimic much better the in vivo blood vessel conditions. In their research on antimicrobial silvercoated poly(ethylene terephthalate) (PET) fabric, it was observed that both experimental procedures gave similar antimicrobial activity results. ${ }^{302}$ However, while the batch experiments suggested that their fabric was toxic to eukaryotic cells due to the release of silver ions, the flow cell tests did not show any toxicity. Thus, appropriate tests need to be done as a function of the applications. For instance, for hip implants, the environment is more static than for vascular grafts so that for the first case a batch colonization experiment would be more appropriate while for the second material a flow cell measurement would be chosen.

In the following subsections, three different strategies for making silver-based biomaterials will be discussed, as well as the different deposition methods and the effect of incorporating silver ions or nanoparticles on the mechanical properties of certain materials.

\subsection{Types of Silver-Containing Biomaterials}

Silver-containing biomaterials could be classified in many different ways. In this Review, we chose to divide them into five categories: (i) metallic silver coatings, (ii) silver-containing nanocomposites, (iii) silver-containing polymers, (iv) surface modification with ionic silver compounds, and (v) hybrid silver materials. The advantages and examples of each type of material are described below.

4.1.1. Metallic Silver Coatings and Silver's Antimicrobial Efficiency. Recent studies employing metallic silver as an implant coating on catheters and orthopedic pins resulted in good antimicrobial activity in vitro, ${ }^{303-305}$ but failed in vivo to prevent bacterial invasion in a significant way. ${ }^{306,307}$ Moreover, despite their low antimicrobial activity found in some in vivo studies, implants coated with metallic silver significantly increased the silver serum level in such a way that, according to Masse et al., ${ }^{306}$ they cannot be ethically used in medicine. One reason for the poor performance of metallic silver coatings during in vivo tests is that silver interacts with the proteins in the blood plasma, which rapidly bind and inactivate the released silver ions, and therefore silver loses its antimicrobial activity (see section 3.1). ${ }^{308}$ Another possible reason for the poor action of metallic silver coatings is that some metallic silver coatings do not actively release silver ions. ${ }^{290,309}$ In fact, it was shown that the rate-determining step for silver release from metallic silver materials depends on the mechanism by which $\operatorname{Ag}(0)$ is oxidized into silver ions as it was already mentioned in section 3.1.1. ${ }^{310}$ Consequently, the rate depends on the film thickness and surface morphology, ${ }^{311}$ and thus on the deposition method (see section 4.2). In a later in vivo study performed by Gosheger and co-workers, ${ }^{312}$ titanium megaendoprostheses coated with metallic silver showed a significant decrease in the infection rate as compared to uncoated prostheses in rabbits. Even though they observed high concentrations of silver ions in animals that received coated implants, they did not observe any pathologic or histological changes. This is understandable because elemental analyses do not differentiate between free silver ions and those inactivated by the binding to proteins. Their clinical study on humans showed similar results for titanium-vanadium megaprostheses coated with elemental silver deposited by galvanic deposition method. ${ }^{313}$ In none of the 20 patients, any sign of argyrosis, the eye condition of argyria caused by improper exposure to silver, was observed as the silver ion concentration in the blood did not exceed $56.4 \mathrm{ppb}\left(56.4 \mu \mathrm{g} \mathrm{L}^{-1}\right)$. In addition, the fibrous tissues surrounding the implant showed a normal ingrowth on the implant without any evidence of toxicity to silver, even though the silver ion concentration within those tissues exceeded $1500 \mathrm{ppb}\left(1500 \mu \mathrm{g} \mathrm{kg}^{-1}\right)$. Nonetheless, and because the use of silver coatings on medical devices is highly controversial and debated, ${ }^{314}$ some studies suggest that materials containing silver ions or AgNPs would have a better antimicrobial activity than metallic silver coatings. ${ }^{315}$

Silver Nanoparticles. AgNPs as well as compounds containing stabilized silver ions have a good silver ion release ability, ${ }^{158,316}$ which makes them more attractive for the development of antimicrobial biomaterials. The size and monodispersity of nanoparticles are apparently not always essential for good activity. In fact, polydispersity can even be beneficial for increasing the duration of the antimicrobial activity based on different release kinetics. ${ }^{317}$ However, in most cases the size of the silver particles was shown to be very important (see section 3.1). This is also demonstrated in an in vitro study ${ }^{318}$ in which a commercially available silver-coated silicone catheter could not completely eradicate any of the strains S. aureus, E. coli, or Propionibacterium acnes. Even though the silver-coated catheter showed some activity at low inocula of $10^{4}$ colony-forming units per milliliter $\left(\mathrm{cfu} \mathrm{mL}^{-1}\right.$ ) and partially killed the bacteria at high inocula of $10^{7} \mathrm{cfu} \mathrm{mL}^{-1}$, the bacterial colonization was regenerated after 3 or 4 days. Two possible reasons for this poor performance are that the catheter surprisingly contained only a little amount of silver as compared to the specifications, and that the silver particles were too big (about $500 \mathrm{~nm}$ in diameter) for efficient silver ion release. ${ }^{318}$ It is also likely that some silver particles were buried too deep inside the silicone material so that they were inaccessible. In addition, AgNPs that are not stabilized tend to aggregate and 
lose their antimicrobial activity and sometimes their biocompatibility. It is therefore essential to protect AgNPs, for example, in a polymeric network. ${ }^{319,320}$ A method to hinder AgNP aggregation can be simply coating the particles with poly(vinyl sulfonate) (PVS). PVS renders them more negatively charged so that they can more easily interact with positively charged surfaces, such as amine-functionalized substrates, and therefore have a better adhesion while staying separated from each other thanks to stronger electrostatic repulsion. ${ }^{315,317}$ The importance of surface charge of AgNPs was already briefly described in section 3.1.

The efficiency of silver-based antimicrobial materials is also highly dependent on their surface morphology and energy. ${ }^{321,322}$ In fact, rougher and more hydrophilic surfaces facilitate the bacterial adhesion in such a way that bacteria are more resistant to antimicrobial agents including silver. In addition, as the total surface energy increases, the bacterial adhesion also increases. The surface roughness and surface energy can be modified simply by using a different reducing agent for the formation of AgNPs from a silver ion solution. ${ }^{321,322}$ In some cases, the addition of silver particles to a material can increase its hydrophobicity and at the same time decrease the adhesion of bacteria to its surface. ${ }^{323}$ Many other factors influence the antimicrobial efficiency of silver. For example, it is highly dependent on its oxidation state, $\operatorname{Ag}(\mathrm{I})$ being a stronger biocide than $\mathrm{Ag}$ (II) or $\mathrm{Ag}$ (III), which are oxidation states also known for silver even though less common than $\operatorname{Ag}(\mathrm{I}) .^{324}$ The method of preparation of the silvercontaining materials has therefore an important impact on its behavior.

4.1.2. Silver-Containing Nanocomposites. Another strategy for producing an antimicrobial implant is based on silver-containing polymers and composites. Titanium-based implants (e.g., TiAlNb, Ti, TiAlV) are commonly used as biomaterials for load-bearing implants due to their well-known biocompatibility, mechanical strength, and corrosion resistance. ${ }^{325-327}$ However, these materials normally have a low antimicrobial activity on their own, if any. To render such an implant antimicrobial, a coating is normally required. Santillan and co-workers ${ }^{89}$ have successfully grown $4 \mathrm{~nm} \mathrm{AgNPs} \mathrm{on}$ $23 \mathrm{~nm}$ titania nanoparticles. This $\mathrm{TiO}_{2}-\mathrm{Ag}$ nanocomposite enables a homogeneous distribution of silver on a substrate and therefore allows a controlled silver release. Coated on a titanium substrate, this $\mathrm{TiO}_{2}-\mathrm{Ag}$ nanocomposite was shown to be bioactive, suggesting that it would allow good cell integration. Moreover, this nanocomposite did not significantly affect the mechanical properties as compared to uncoated titanium, ${ }^{89}$ which suggests that it is an interesting coating for medical implants. The combination of the antimicrobial activities of silver and titanium oxide has also been previously reported to prevent infectious contamination by inserting such nanoparticles in facial masks without provoking any evidence of inflammation for the users. ${ }^{103}$ On another hand, Huang et al. ${ }^{328}$ propose a silver-containing tantalum nitride ( $\mathrm{TaN}-\mathrm{Ag}$ ) material as a coating for titanium-based implants. TaN-Ag coatings considerably decrease the bacterial adhesion of $S$. aureus as compared to uncoated titanium and silver-free TaN. TaN-Ag coating has also shown excellent biocompatibility as it allowed the cell attachment of human gingival fibroblasts in an even more efficient way than uncoated titanium. ${ }^{328}$ Another material, the silver-doped porous $\mathrm{P}_{2} \mathrm{O}_{5}-\mathrm{SiO}_{2}$ monoliths that were prepared by a sol-gel method, had a stable silver release in water at $30{ }^{\circ} \mathrm{C}$ over a period of 28 days, thus demonstrating that silver-containing nanocomposites can provide a slow release of silver ions. ${ }^{329}$ This 4-week period would offer a good short-term protection for the critical period ${ }^{330}$ after the surgery. However, even though the expected time for an orthopedic implant to stay in the human body is between 10 and 15 years, ${ }^{331}$ in the literature, no long-term studies are described for silver-based implant materials according to our knowledge.

4.1.3. Silver-Containing Polymers. An alternative method to enhance the properties of silver-based materials is the incorporation of silver ions or nanoparticles into hydrogels. Many groups have successfully developed such hydrogels that allow a slow and controlled release of silver ions, ${ }^{309}$ as, for instance, a PET surface modified by silver ion implantation, ${ }^{332}$ silver nitrate-catalyzed polyacrylamide gels, ${ }^{333}$ and PET-based hollow fibers containing silver particles. ${ }^{34}$ Slow and controlled silver release could also be achieved from polymers. As an example, Damm et al. $^{335}$ could fabricate a material that maintains a constant antimicrobial efficiency over long periods of time. In fact, they synthesized a polyamide 6-silver nanocomposite that, even after 100 days immersed in water, still releases silver at the same rate demonstrating a zero-order release. ${ }^{315}$ The silver release of some silver-containing materials is given in Table 7 .

Table 7. Examples of Time Needed To Reach the Maximum Silver Concentration (Plateau) for Different Materials ${ }^{a}$

\begin{tabular}{|c|c|c|c|c|}
\hline material type & medium & $\begin{array}{l}\text { temp } \\
\left({ }^{\circ} \mathrm{C}\right)\end{array}$ & $\begin{array}{l}\text { time to reach } \\
\text { plateau }\end{array}$ & ref \\
\hline Ag-doped $\mathrm{P}_{2} \mathrm{O}_{5}-\mathrm{SiO}_{2}$ monoliths & water & 30 & $\begin{array}{l}\text { more than } \\
28 \mathrm{~d}\end{array}$ & 329 \\
\hline polyamine $6 / \mathrm{Ag}$ nanocomposite & water & rt & $\begin{array}{l}\text { more than } \\
100 \mathrm{~d}\end{array}$ & 335 \\
\hline $\begin{array}{l}\text { Ag-doped perfluoropolyether- } \\
\text { urethane }\end{array}$ & PBS & 37 & $1 \mathrm{~d}$ & 336 \\
\hline $\begin{array}{l}\text { Ag-doped perfluoropolyether- } \\
\text { urethane siloxane film }\end{array}$ & PBS & 37 & $4 \mathrm{~d}$ & 337 \\
\hline Ag-containing PET hollow fibers & PBS & 37 & $2 \mathrm{~d}$ & 334 \\
\hline PVC silver zeolite & $\begin{array}{l}\text { sterile } \\
\text { urine }\end{array}$ & 37 & $5 \mathrm{~d}$ & 338 \\
\hline AgNP in acrylic resin & water & rt & $2 \mathrm{~d}$ & 339 \\
\hline
\end{tabular}

${ }^{a}$ PBS stands for phosphate buffered saline, which is a commonly used buffer solution ( $\mathrm{rt}$, room temperature; d, day).

It is often easy to trap silver ions or AgNPs within a polymer matrix. For many resins, it can be performed by immersing the polymer in a silver-containing solution and then adding a reducing agent to form AgNPs. The porosity allows the diffusion of silver ions within the interior of the polymer matrix in such a way that silver ions can interact with the functional groups of the polymer, as depicted in Figure $18 .{ }^{340}$ The reductant then also diffuses through the matrix and induces the formation of well-dispersed AgNPs on the surface as well as in the interior of the resin. ${ }^{340}$ This kind of impregnation with silver has shown advantages as compared to simple coating, such as a slower silver release for nanoparticles that are located deeper inside the matrix and the protection of the whole biomaterial, not only of one surface.

Travan and co-workers ${ }^{341}$ obtained a noncytotoxic hydrogel that successfully killed Gram-positive bacteria including $S$. epidermidis and $S$. aureus, which are major concerns for catheters and prosthetic implant infections, respectively, and Gram-negative bacteria, such as E. coli. They used a lactosesubstituted chitosan, the so-called Chitlac, to embed AgNPs in 


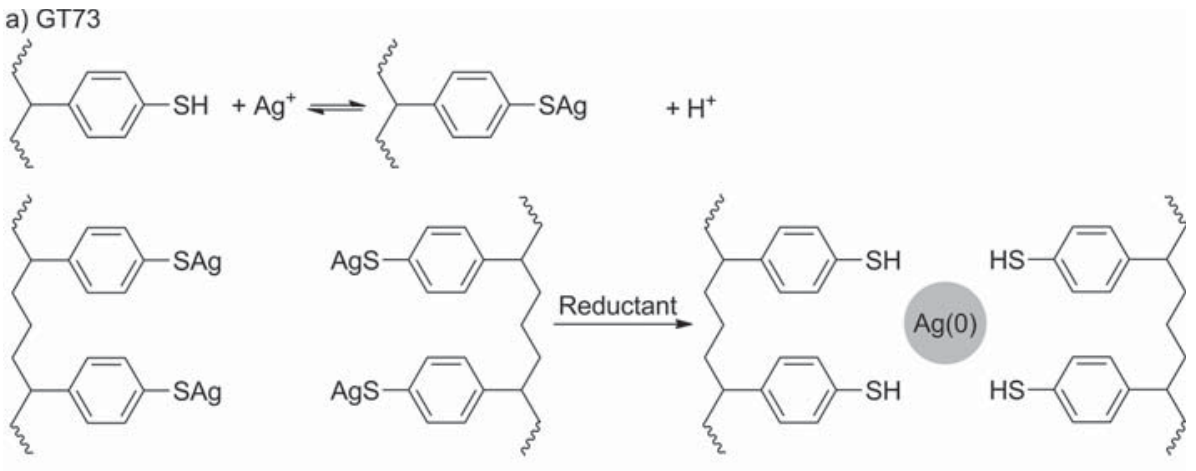

b) IRC748

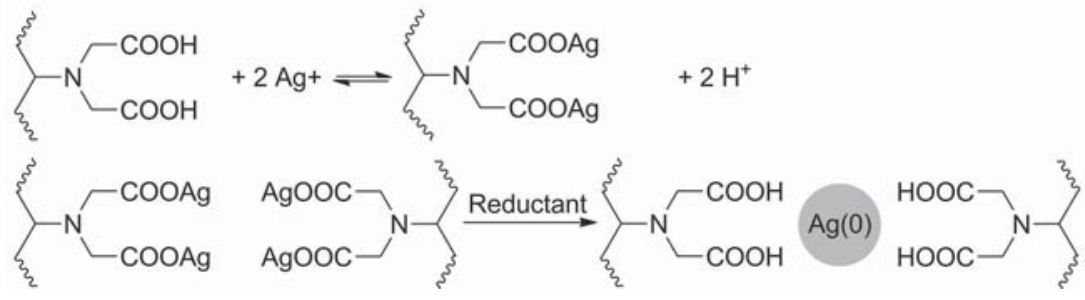

Figure 18. Proposed model for the formation of silver particles around and/or within the bead macrostructure of two different resins: (a) GT73 and (b) IRC748. Adapted with permission from ref 340. Copyright 2007 John Wiley and Sons.

a very disperse manner, preventing their aggregation over long time periods (more than 90 days). Their silver-containing hydrogel allows the AgNPs to contact the cellular membranes without being taken up by the cells. It therefore showed specific toxicity toward bacteria, but not toward eukaryotes because eukaryotic cell membranes are considerably less sensitive to silver. In addition, this Chitlac hydrogel only very slowly releases silver ions in saline solutions, so that it is considered to be noncytotoxic for humans. ${ }^{341}$ In a later work, Travan and coworkers $^{342}$ demonstrated the efficiency of this hydrogel as an antimicrobial coating for methacrylic thermosets, suggesting its possible application to dental and orthopedic implants.

In silver-containing hydrogels, the silver release rate depends mostly on the rate of water diffusion in the polymer matrix. ${ }^{309}$ This can be controlled by the crystallinity of the polymer matrix, ${ }^{343}$ the filler type, ${ }^{344}$ the silver particle morphology, ${ }^{345}$ the silver concentration, the silver grain specific surface area, the physical state of the composite, and the physical changes due to the incorporation of silver. ${ }^{309}$ These factors can be varied to increase the antimicrobial activity while decreasing the toxicity of silver. A slow release of silver ions in such systems can also be controlled by the adhesion of the silver ions or nanoparticles. In other words, strongly attached silver to the polymer will be released slower than weakly attached silver. ${ }^{309}$

In an in vivo study, Boswald and co-workers ${ }^{346}$ demonstrated a good biocompatibility for a silver-impregnated catheter. They placed normal and silver-containing poly(urethane) (PU) and silicone tubes in rats and observed the incidence of abscesses due to this intervention. For PU tubes, there was no significant difference between the silver-impregnated and the silver-free tubes. On the other hand, silver-impregnated silicon catheters showed a considerable decrease in the abscess incidence as compared to the normal silicon tubes. Histological tests of the specimens receiving a silver-containing catheter did not show the presence of silver particles in any of the subcutaneous, muscular, or peritoneal tissues. ${ }^{346}$ Clinical studies using an urinary catheter coated with a hydrogel embedding silver ions also showed very promising results for the use of such medical devices. In fact, a reduction of infection cases was observed as compared to uncoated catheters, ${ }^{303,347}$ which also results in a significant reduction in medical costs. ${ }^{303}$ Plowman and coworkers $^{348}$ developed a model for evaluating the potential benefits of the routine use of silver-coated urinary catheters. They compared the additional medical costs due to infection occurrence to the costs of production of silver-coated catheters, considering also their efficiency of about $32 \%,{ }^{347}$ and they concluded that their routine use would significantly reduce medical costs. ${ }^{348}$

Because textiles are recognized as an ideal medium for microbial development, AgNPs are proposed as a component in fabrics such as polyester or cotton fabrics. Some works demonstrate that embedding silver within a textile, similarly to polymers, can stabilize the AgNPs in such a way that they can retain their biocidal activity for a longer time and thus prevent microbial development. ${ }^{349,350}$ This is of great interest for the fabrication of antimicrobial bandages, as an example.

Biodegradable Coatings. The use of biodegradable coatings containing silver ions might also be beneficial for a controllable silver release. For example, it is possible to dope phosphatebased glasses with silver to prevent urinary tract and wound dressing infections. The release of silver ions was shown to be dependent on the degradation of the glass. ${ }^{324,351}$ Good biocompatibility as well as antimicrobial activity have also been demonstrated for bone cements loaded with AgNPs, ${ }^{352,353}$ such as HA cements, which are commonly used in implants to promote bone ingrowth for bone regeneration, ${ }^{353}$ and gelatin nanofibers or films loaded with AgNPs. ${ }^{354,355}$ Moreover, flexible and biodegradable silver-containing nanocomposites offering prolonged antibacterial protection were developed as good materials for dental or reconstructive bone surgery. ${ }^{356}$

Liposomes were also used to trap silver within a polymer coating. In fact, Malcher and co-workers ${ }^{357}$ could make an antimicrobial composite containing liposomes filled with silver nitrate using a sonication technique. They first filled the liposomes with silver nitrate solution, and then, with the help of poly(L-lysine) (PLL), they forced these liposomes to aggregate. 
These aggregates were then incorporated into a multilayer PLLpolyanion film. This coating was shown to be very efficient against E. coli invasion.

Qureshi et al. ${ }^{358}$ have also developed a bioabsorbable coating for implants. This coating, made of poly(L-lactic acid) (PLLA), contains about $7 \times 10^{5} \mathrm{ppb}\left(7 \times 10^{5} \mu \mathrm{g} \mathrm{kg}^{-1}\right)$ cellulosestabilized AgNPs. It demonstrated very good in vitro antimicrobial activities against both Gram-negative and Grampositive bacterial strains. During the first day, the silver release reached $177.4 \mathrm{ppb}\left(177.4 \mu \mathrm{g} \mathrm{L}^{-1}\right)$, which did not affect the in vitro viability of human HeLa cells. ${ }^{359}$ On a longer time scale, this biomaterial releases silver ions at a slower rate.

In short, the embedment of silver ions or AgNPs within a polymer stabilizes the silver compounds and allows a slower release and therefore a longer antimicrobial efficiency.

4.1.4. Surface Modification with Ionic Silver Compounds. Balazs et al. ${ }^{360}$ clearly demonstrated that a simple surface modification can significantly prevent and eliminate bacterial adhesion on medical devices. In fact, the chemical modification of poly(vinyl chloride) (PVC), which is commonly used in endotracheal tubes, with sodium hydroxide and silver nitrate prevented any bacterial invasion for more than 72 h. ${ }^{360,361}$ A second promising result was provided by Berra et al. ${ }^{362}$ who observed in a clinical study that PU tubes that are internally coated with silver sulfadiazine prevented the occurrence of bacterial colonization without any observable negative effects. ${ }^{362}$

Another strategy for surface modification with silver involves the use of coordination polymer networks and polymorphs to capture and slowly release silver ions. (Iso)nicotinic acid derivatives (of type 21) have been intensively studied in our group for this purpose, ${ }^{363-369}$ thanks to their flexible backbone and their good biocompatibility. ${ }^{370,371}$ This type of coating was shown to efficiently prevent implant infection on dental devices. ${ }^{372}$ Their tunable solubility, stability, and antimicrobial efficiency make coordination polymer networks interesting "smart" coatings for biomaterials. They can be used to modify gold and titanium surfaces with, for example, [Ag(ethane-1,2diyl diisonicotinate) $\left.\mathrm{NO}_{3}\right]_{n}(22)$, as depicted in Figure 19, using disulfide $\mathbf{2 3}$ as anchor molecule. This coating shows a very good antimicrobial activity against a broad spectrum of pathogens while having a low toxicity. In vivo studies show that this coating was efficient against the implant infection strains S. epidermidis and S. aureus over 21 days, independently of whether they carried the biofilm-forming ica-gene or not. We also observed that, even though there is formation of a biofilm, the antimicrobial action of silver was not affected. This property seems to be related to a mechanism involving the binding of silver to the amino acids containing thiol groups, including the respiratory chain and citric acid cycle enzymes, leading to their inactivation. Another effect of silver in bacteria is the formation of hydroxyl radicals, leading to DNA damages, ${ }^{207}$ as discussed in section 3.1.

A similar strategy has been developed by Amalric and coworkers. $^{373}$ They propose a nanosized silver-containing mercaptododecylphosphonic acid coating obtained by a simple chemical surface modification. Despite its low silver concentration, this self-assembled monolayer coating was shown to reduce bacterial adhesion and biofilm formation by more than $99.9 \%$ on both titanium and stainless steel samples. ${ }^{373}$

In short, the surface modification of implants with silver compounds offers an interesting strategy to prevent infections.

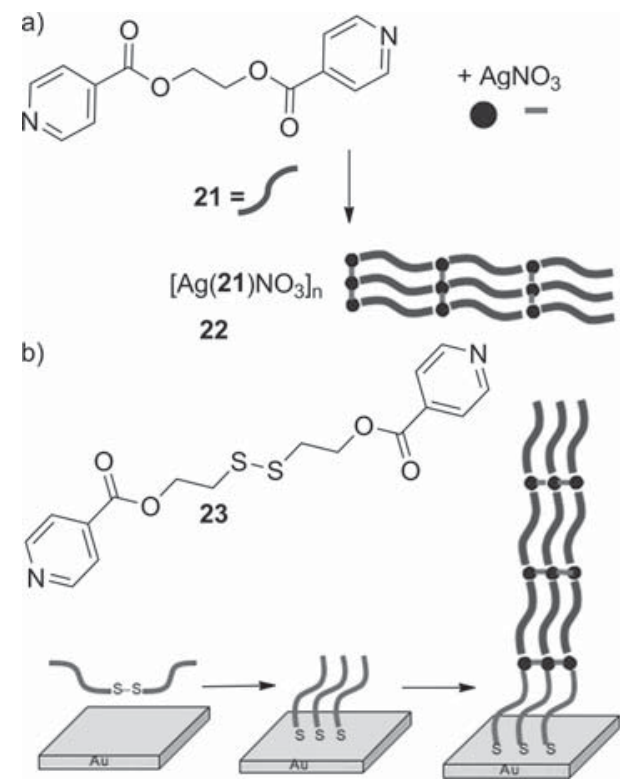

Figure 19. Synthesis and surface treatment with the silver coordination compound $\left[\mathrm{Ag}(21) \mathrm{NO}_{3}\right]_{n}$ (22). (a) Formation of the silver coordination compound 22 and (b) its deposition on $\mathrm{Au}(\mathrm{III})$ plates using the disulfide $\mathbf{2 3}$ as anchor molecule for the surface modification. Possible modes of AgNPs internalization. Adapted with permission from ref 207. Copyright 2010 American Society for Microbiology.

4.1.5. Hybrid Silver Materials - Synergistic Effects. Many researchers propose the combination of silver with another antimicrobial agent to enhance its biocidal efficiency. Thanks to this synergy effect, these so-called hybrid materials either provide different ways to kill bacteria, or one component can simply enhance the effect of the other.

An example of hybrid antimicrobial systems is the dual action of a hydrophobic material with the release of silver ions, which have recently been suggested as suitable antimicrobial coatings for medical devices. ${ }^{336}$ This was also demonstrated with silverdoped perfluoropolyether-urethane siloxane thin films deposited on a glass substrate. ${ }^{337}$ The hydrophobic sites of this coating are proposed to protect the action of silver ions. In fact, the hydrophobic sites seem to inhibit the attachment of ionic species that might reduce the available silver ions located on the surface or form silver salt deposits (see Figure 25, section 5.5), which would considerably reduce the biocidal action of silver, as shown in section 3.1. As perfluoropolyether-urethane siloxane protects silver from deactivation, the released silver ions protected these hydrophobic sites against $S$. epidermidis attachment and colonization, ${ }^{337}$ even though this strain was shown to colonize preferentially hydrophobic sites over hydrophilic sites. ${ }^{374}$ The silver-doped perfluoropolyetherurethane siloxane thin films showed very good antimicrobial activity against both $S$. epidermidis and A. baumannii biofilm formation. ${ }^{337}$ In addition, the introduction of fluorocarbon and AgNPs increases the biostability of these polyether urethane derivatives, ${ }^{375,376}$ which makes them more attractive for medical uses.

A second example for hybrid silver materials was mentioned earlier in section 2.3.1. Eby et al. ${ }^{140}$ proposed the combination of silver with lysozyme, an antimicrobial enzyme for making an antibacterial and self-cleaning coating for surgical instruments. They also realized that the use of lysozyme can be beneficial for 
the design of silver-containing coatings. In fact, lysozyme can be used as a reductant and stabilizer during the synthesis of AgNPs in methanol. The nanoparticle synthesis did not affect the electrochemical properties of the enzyme, which are necessary for the electrophoretic deposition to be performed without the addition of other electrolytes. Eby et al. ${ }^{140}$ also observed that the presence of lysozymes increases the adsorption strength of AgNPs onto stainless steel surfaces. In addition to the biocidal activity of lysozyme, this coating would also allow a release of silver ions. Even though the antimicrobial efficiency still requires improvement for such coating, this lysozyme-silver hybrid coating is very promising for the development of antimicrobial robust and self-cleaning medical instruments. ${ }^{140}$

Because chitosan has also been shown to have antimicrobial activity against a broad spectrum of bacterial strains, ${ }^{377}$ it is not surprising to find many studies combining both silver and chitosan. For example, $\mathrm{Fu}$ et al. $^{378}$ proposed a coating consisting not only of silver and chitosan, but also of heparin as an anticoagulant. The nanosilver was deposited on PET, which is a material commonly used for cardiovascular medical devices. This coating was fabricated by successive layers of chitosan-silver nitrate complex and heparin, and the nanoparticles were synthesized by the reduction of silver ions by ascorbic acid. They proposed this material to eradicate two major problems encountered in cardiology: cardiovascular infections, as it is one of the most commonly occurring postimplantation infections, and thrombogenicity, that is, the formation of a clot due to the contact of a material with blood. Indeed, the strong anticoagulating properties of chitosan and heparin ${ }^{379}$ were not affected by the presence of silver. This coating is therefore not only easy and cheap to synthesize, but it has also good antimicrobial, anticoagulating, and biocompatible properties. $^{378}$

Other studies have demonstrated the strong antimicrobial activity of the silver-chitosan combination against both Gramnegative and Gram-positive bacteria, including the MRSA. ${ }^{380}$ This combination was shown to also have a good biocompatibility in vitro and in vivo so that its use has been proposed for medicine, ${ }^{381}$ for example, in burn and wound dressings, ${ }^{382-385}$ and in food packaging. ${ }^{315,381,386}$ Huang et al. $^{380}$ offer an explanation for the high efficiency of silverchitosan material. They suggest that their combined actions are synergistic rather than being simply additive. Chitosan renders the bacterial cell membrane more permeable in such a way that silver ions can penetrate more easily the bacteria and kill them. This theory is confirmed by their observation that Gramnegative bacteria, having a high intrinsic permeability barrier, that is, being very impermeable to exchanges with the environment, are more affected by this synergy action of silver and chitosan than Gram-positive bacteria. They also observed in vivo that a chitosan-silver bandage offers a very good treatment against bacterial infection in burns. ${ }^{380}$ The exact molecular mechanism of action and the structure of this hybrid material still remain unclear.

Cao and co-workers ${ }^{387}$ suggested that the good antimicrobial activity against $S$. aureus and $E$. coli and the low toxicity to eukaryotes, which they observed for their titanium matrix containing AgNPs, is due to a combined action of silver and titanium. They explained this difference in biological activity against prokaryotic and eukaryotic cells by a microgalvanic effect created by silver and titanium, which is affecting the proton active transport of the bacterial plasma membrane, making the material more toxic to bacteria.
In short, silver-containing compounds can benefit from the combinations with other materials to increase their antibacterial efficiency and/or their stability.

\subsection{Deposition Processes}

For many of the antimicrobial coatings shown so far, one characteristic that is often forgotten for an antimicrobial coating to be commercially applicable is their ability to be easily and economically deposited on an implant. The choice of the coating method is of major importance as it must ensure a good adhesion of the coating components on the substrate and maintain the proper chemical compositions, structure, and bioactive properties. ${ }^{388}$ Thus, there is no "the one and only" technique that can be used for all materials and applications. Examples of coating techniques are ion implantation, ${ }^{332}$ slipcasting, glue-bonding, isostatic pressing, rolling, electrochemical deposition, casting, and plasma sputtering. ${ }^{389-392}$ Some of the most commonly used techniques require heating of the coating to high temperature. However, as demonstrated by Taylor et al., ${ }^{393,394}$ nanocrystalline silver is unstable at elevated temperatures, resulting in considerable changes in its physical and chemical structure, which is considered to play a major role in its antimicrobial activity. ${ }^{393}$ In fact, the silver release in polymer-based dressings treated at temperatures above $75{ }^{\circ} \mathrm{C}$ was considerably reduced in such a way that its antimicrobial activity is considerably affected. ${ }^{394}$ Thermal deposition processes are therefore not desirable for many silver-containing coatings. Nonetheless, Ando and co-workers ${ }^{395}$ have demonstrated that a silver-containing calcium phosphate layer could be thermally sprayed on titanium substrates. This coating also shows interesting antimicrobial activity, as well as a good biocompatibility. A silver-doped diamond-like carbon coating could also be generated and deposited using a pulsed filtered cathodic vacuum arc. This surface showed excellent hemocompatible and antimicrobial efficiency so that it was recommended for implants and medical devices. ${ }^{396}$

Many other deposition techniques have been proposed and tested for medical devices with coatings that are heat-sensitive. Galvanic deposition was used to coat metal-based prostheses with elemental silver. The in vivo tests showed that this coating remained intact after a long period of about 19 months. ${ }^{313}$ The deposition of a metallic material on a polymer is more complicated than on another metal because metals and polymers have different surface energies, leading to a weaker adhesion on polymers. Thus, an electroless plating process has been proposed as a cheap and easy method to deposit silver on poly(ether urethane) (PEPU) without the use of vacuum. ${ }^{397,398}$ Nonetheless, plasma treatment is still recommended because it greatly improves the silver adhesion on the polymer. Better adhesion results in the formation of a higher amount of small silver clusters instead of only few big clusters with weak adhesion. ${ }^{398}$ As smaller nanoparticles exhibit a higher dissolution rate, the antimicrobial efficiency of this (PEPU) silver composite increases. ${ }^{397}$ On the other hand, plasma treatment results in some chemical changes to the polymer surface such as and an increase in carbonyl, alcohol and alkene groups. ${ }^{399}$ The presence of additional $\mathrm{C}=\mathrm{O}$ groups probably explains the better adherence of silver to plasma-treated polymer surfaces. ${ }^{398}$

It is important to keep in mind that the morphology, and therefore the silver release efficiency, of metallic silver coatings can be very dependent on the deposition technique used and its parameters. To coat the inside of silica capillaries with silver, 
Wen et al. ${ }^{400}$ used the wet chemistry deposition method. They realized that as the volume of silver nitrate as precursor for the silver coating increases, the grain size and the surface roughness of the coating also increase. ${ }^{400}$ When the silver film was fabricated by first producing AgNPs by laser ablation of microparticle aerosols and then depositing them by supersonic jet deposition, the morphology and density can be controlled. Increasing the annealing temperature produces bigger grains, ${ }^{401}$ and rapid heating produces films of higher density. ${ }^{402}$

A well-adhered silver layer was successfully deposited on PU and polyethylene substrates during a magnetron deposition process combined with a neutral atom beam plasma source at $70{ }^{\circ} \mathrm{C} .{ }^{403}$ The resulting substrates have good antimicrobial activities and low cytotoxicity. However, even though it is successful for soft polymer substrates, this combined deposition technique is not as efficient for hard polymer substrates. In fact, coatings on silicon for example exhibit less adhesion and become more cytotoxic as compared to the soft polymers. ${ }^{403}$ Co-sputtering of silver together with the embedding mineral, such as HA, was also shown to be effective to create a nanocomposite coating with well-dispersed silver. ${ }^{353}$

Figure 20 lists the surface modification techniques most published for coating biomaterials. As it can be observed, the

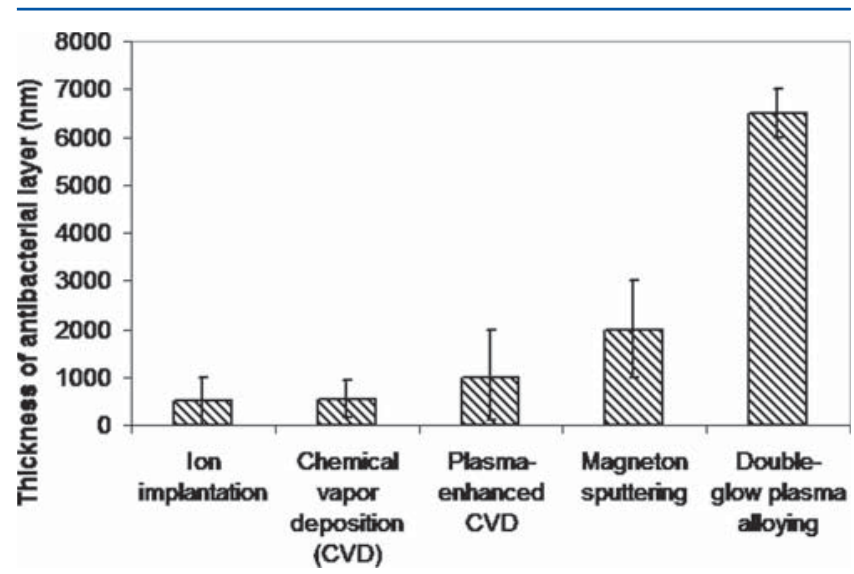

Figure 20. Effective thicknesses of the four most published surface modification techniques in addition to double-glow plasma alloying for achieving antibacterial surfaces. The bars indicate the maximum and minimum thicknesses as published. Possible modes of AgNPs internalization. Adapted with permission from ref 404. Copyright 2011 Elsevier.

layer thickness is highly dependent on the chosen deposition technique. As pointed out by Dong et al. ${ }^{404}$ for a long lasting antimicrobial coating, it is often preferable to have a thick coating as it might be subjected to dissolution and wear forces. They also demonstrated that the silvering of a stainless steel surface could be effectively achieved by a double glow plasma alloying technology, resulting in a good antibacterial material. This silvering resulted in a harder stainless steel material, which in turn improved the wear resistance. This material can therefore be beneficial for load-bearing implants as they are highly exposed to surface damage, to improve the bioactivity duration and reduce the contamination by the implant residues. Reactive pulsed laser deposition (PLD) could also be used to fabricate nanostructured $\mathrm{Ag}_{4} \mathrm{O}_{4}(\mathrm{Ag}(\mathrm{I})-\mathrm{Ag}(\mathrm{III})$ oxide) films with good antimicrobial activity and allows to control the crystallinity, the grain size, and the morphology of these films. ${ }^{405} \mathrm{Ag}_{4} \mathrm{O}_{4}$ consists of two monovalent and two trivalent silver ions per molecular crystal, forming a distorted facecentered cubic arrangement of silver ions. ${ }^{406} \mathrm{Ag}_{4} \mathrm{O}_{4}$ has already been suggested for water treatment as it was shown to kill $E$. coli and Streptococcus faecalis. ${ }^{407}$

Other methods for making silver-containing coatings, such as the sol-gel method for colorless silver-containing silica glass powders for dental implants ${ }^{408}$ or multilayered silver-containing $\mathrm{TiO}_{2}$ films, ${ }^{409}$ are effective to embed silver ions and gradually release them.

As it was just presented, the choice of the deposition technique is of major importance for obtaining efficient antimicrobial coatings for implants.

\subsection{Mechanical Properties}

One major concern about inserting silver compounds or nanoparticles within biomaterials and coatings is that it might affect its mechanical properties. In some cases, the silver component can be beneficial for the overall material. For example, in addition to its good antimicrobial activities, the dental acrylic resin embedding AgNPs developed by Kassaee et al. ${ }^{339}$ shows slightly improved flexural strength and modulus as compared to the commonly used dental acrylic resin. ${ }^{339}$ The use of AgNPs in dental acrylic resins is therefore favorable as compared to the incorporation of other silver-containing compounds such as $\mathrm{Ag}-\mathrm{Z}$. In fact, even though $\mathrm{Ag}-\mathrm{Z}$ has shown good antimicrobial activity against bacteria, as shown in section 3.1.3, and a well-controlled silver release, ${ }^{338,339,410}$ the incorporation of more than 2.5 wt \% Ag- $\mathrm{Z}$ in the acrylic resin resulted in significant deterioration of its mechanical properties. ${ }^{411}$ On the other hand, the introduction of Ag-Z in PVC composites, even at a concentration up to $20 \mathrm{wt} \%$, did not result in any significant changes in tensile strength, elongation at break, viscosity, or thermal properties. Only the modulus is increased with the increased concentration of zeolite in PVC, making the composite stiffer, but not enough to influence its processability and formability. Moreover, its antimicrobial effect could be activated with diluted acid, ${ }^{338}$ which is of interest because bacteria tend to acidify the medium around them.

Good mechanical properties were also observed for Ti-based alloys containing silver. ${ }^{412-415}$ For example, the addition of silver and copper improves the mechanical strength of titanium alloys without affecting the corrosion resistance, making this $\mathrm{Ti}-\mathrm{Ag}-\mathrm{Cu}$ alloy very attractive for dental applications. ${ }^{416}$ Another example of improved mechanical properties is that the swelling properties of some hydrogels could also be altered by incorporating silver ions. This is the case for a silver-containing thermoplastic nanofibrous hydrogel that shrinks during water uptake, while normally hydrogels swell. This unusual hydrogel can therefore have interesting applications for wound dressings. ${ }^{417}$

Despite these promising examples, silver integration does not always lead to better mechanical properties. Thus, Radheshkumar and Münstedt noticed a significant decrease in toughness and elongation strength when $2 \mathrm{wt} \%$ silver powder $\left(2 \times 10^{7} \mu \mathrm{g}\right.$ $\mathrm{kg}^{-1}$ ) having a specific surface area of $0.78 \mathrm{~m}^{2} \mathrm{~g}^{-1}$ is introduced into polyamide, even though the silver powder was well dispersed. As the silver concentration increases, the mechanical properties deteriorate. $^{418}$

In short, we have observed that the addition of silver, in either metallic bulk, ionic, or nanoparticular form, is generally beneficial for improving the antimicrobial properties of biomaterials and that the antimicrobial activity of silver can be amplified by the synergistic effects of several agents. 
However, the properties of an implant material, such as its mechanical properties, can be altered by the addition of silver in any form, and the deposition technique, in the case of antimicrobial coatings, can also influence its efficiency. Finally, when treating an implant with silver, it is wise to avoid the generalization of effects and the transfer of properties from one system to the other, but rather test and confirm all derived properties with regard to the applications, in vitro and in vivo, as the properties of silver-containing materials can significantly vary depending on the environment.

\section{BIOCOMPATIBILITY OF SILVER}

Medical devices need to possess, next to being antimicrobial, a second important property: they must be biocompatible. Indeed, biocompatibility has become the central request for medical applications of materials and devices before being placed in the body. The determination of cytotoxicity implies that a material has to be (i) free of damaging effects on the host, (ii) able to elicit a beneficial host-response for optimal function, and (iii) safe, which deals with the exclusion of deleterious effects of a biomaterial on the organism itself.

As discussed in section 4, manufacturers are increasingly looking at silver as a promising solution to prevent infections in the medical field. ${ }^{419}$ Free silver ions, because of their low cytotoxicity to mammalian cells ${ }^{420-423}$ and broad-spectrum antimicrobial activity, have gained attention in a wide range of applications in the healthcare sector with a high degree of commercialization. Silver coatings of foreign materials have been safely used in medicine for many years and are proven to reduce infection rates of medical devices in vitro and in vivo. ${ }^{346,347,420,424-430}$ They are used in burn wound dressings, orthopedic and dental implants, bone prostheses, surgical instruments, intravascular catheters, prosthetic heart valves, cardiac pacemaker, and contraceptive devices. ${ }^{431,432}$ These devices interact with the human body at different levels and places, and we classified them according to their contact levels with the human body as discussed in the following subsections.

\subsection{General Routes of Silver Exposure}

The human body has several semiopen interfaces such as the respiratory tract, the gastrointestinal tract, and the skin (Figure 21), which can get exposed to three major product categories: food, consumer products, and medical products.

Inhalation of vapors, gases, mists, or particulates is one of the major routes of entry of silver compounds into the body. Once inhaled, silver compounds, and more specifically AgNPs, are

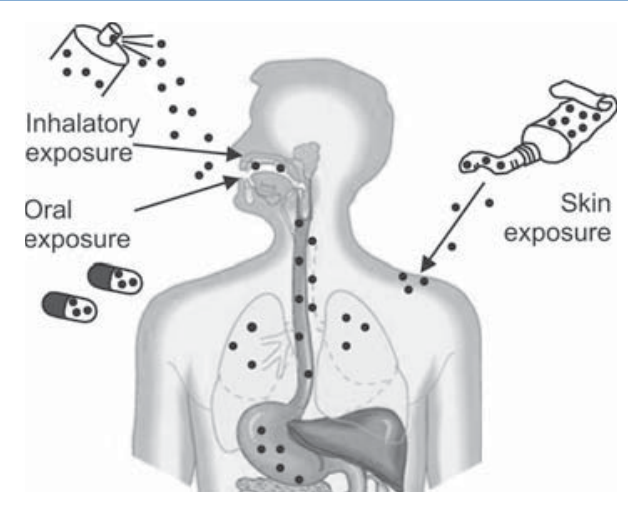

Figure 21. The three main exposure sites of the human body for the contact with silver compounds. either inhaled or deposited in the respiratory tract, causing damage through direct contact with tissues, or are diffused into the blood through the lung-blood interface. The respiratory tract is, apart from the skin, the only organ system in direct contact with the environment. Nasopharyngeal (ciliated epithelium and mucous glands), tracheobronchial (consisting of trachea, bronchi, and bronchiols) and pulmonary acini (small bronchioles with alveoli connecting to pulmonary capillaries) are components of the respiratory tract. ${ }^{433}$ The distribution and disposition of silver particles in the respiratory tract depend on various factors including particle size and breathing force. ${ }^{434}$ Indeed, particles with a size of $5-30 \mu \mathrm{m}$ are deposited in the nasopharyngeal region, whereas $1-5 \mu \mathrm{m}$ particles are deposited in the tracheobronchial region. For AgNPs with a size of less than $1 \mu \mathrm{m}$, deposition occurs in the alveolar region by diffusion and Brownian motion resulting in a deep penetration of AgNPs into the lungs, followed by the absorption into the blood system and distribution into the organs. ${ }^{435}$

Ingestion of silver compounds is the second most common route of exposure. Indeed, silver may enter the body through the mouth, throat, or digestive tract after ingesting silvercontaining food, water, or medication. The body of an adult normally contains ca. $29 \mu \mathrm{g} \mathrm{kg}^{-1}$ of silver. Our daily oral intake of silver is $20-80 \mu \mathrm{g} \mathrm{d}^{-1}$ of which approximately $10-20 \%$ are absorbed $^{436}$ by the gastrointestinal tract, mainly by the duodenum and small intestine. Insoluble silver compounds in the fluids of the gastrointestinal tract (stomach, small, and large intestines) are generally excreted, whereas other silver compounds that are soluble are absorbed through the lining of the gastrointestinal tract. They are then transported by the blood to internal organs (see section 5.3). ${ }^{437}$

Dermal exposure represents the third important absorption route for silver. ${ }^{203}$ The skin is, in terms of weight, the largest organ of the body. It provides a barrier between the environment and other organs (except the lung and eyes) and is a defense against many chemicals. Silver can enter the body through the skin when it is in direct contact with solutions containing silver compounds, such as those used in photography, or when in contact with silver wound dressings as will be discussed in the following section in the context of exposure to silver medical products.

\subsection{Exposure to Silver-Containing Medical Products}

As discussed in section 4, silver is commonly used in medical devices such as in burn wound dressings, wound care products, catheters, orthopedic and dental implants, heart valves, and bone cements, which are typically in direct contact with skin, blood, and/or bones. In this subsection, we will discuss the silver exposures originating from (i) burn wound dressing with dermal contact, (ii) medical devices with bone contact, such as orthopedic and dental implants, or bone filling products, and (iii) implants with permanent blood contact such as catheters, stents, or shunts.

5.2.1. Dermal Contact: Burn Wound Dressings. Benefits from silver therapeutics and silver-releasing dressings on wound care and regeneration products with regard to their antimicrobial efficacy have been well studied (see also examples given in section 4). Yet, little is published on how the released silver ions influence the wound bed, or how they are metabolized and deposited in the tissue. Wound dressings are especially used on burned skin, and dermal absorption of silver and elevated silver levels in plasma and urine were observed after treatment with such dressings. ${ }^{438}$ 
Different processes are implicated in the burn wound repair such as inflammation around the site of injury, angiogenesis and the development of granulation tissue, repair of the connective tissue and epithelium, and remodeling. However, wound repair is slowed due to the bacterial colonization that prolongs the inflammatory response, which may also damage the surrounding tissue. ${ }^{439}$ To support the wound healing by maintaining a microbe-free and a moist wound healing environment, ${ }^{440}$ silverbased dressings are often used to prepare the wound for healing. Over the past years, there was a rapid increase in the number of silver dressings. Several silver products, such as silver nitrate, silver [(4-aminophenyl)sulfonyl $]$ (pyrimidin-2-yl)azanide better known as silver sulfadiazine (SSD), ${ }^{441}$ and introduced as gold standard by Fox in 1968 (Figure 22), ${ }^{442}$ SSD/chlorhexidine, SSD with cerium nitrate, and a SSD impregnated lipidocolloid wound dressing ${ }^{205,441,443}$ have been used for topical burn wound therapy.

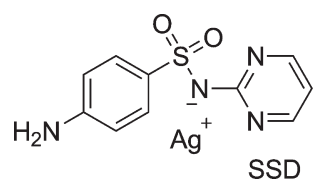

Figure 22. Structure of silver sulfadiazine.

Previously reported effects of silver nitrate on burn wounds were based on clinical studies and observations showing that concentrations exceeding $1 \%\left(1 \times 10^{7} \mu \mathrm{g} \mathrm{L}^{-1}\right)$ of silver nitrate were toxic to tissues. Hidalgo et al. ${ }^{444}$ showed in vitro that, at direct exposure, $\mathrm{AgNO}_{3}$ greatly inhibits the growth of bacteria starting at concentrations as low as a $0.0055 \%$ solution of $\mathrm{AgNO}_{3}\left(5.5 \times 10^{4} \mu \mathrm{g} \mathrm{L}^{-1}\right)$ in purified water, a level approximately 100 times lower than those used in clinical practice. Although ionic silver is said to have a beneficial effect of decreasing wound surface inflammation, ${ }^{440,445}$ some studies have recently shown a potential silver toxicity of SSD on various host cells and a delayed wound-healing process. ${ }^{446-449,449,450}$ Such contradictory studies reflect the literature. Thus, in vitro and in vivo studies do not always fully concur with each other, ${ }^{451,452}$ and in vitro cytotoxicity alone cannot predict the in vivo toxicity. Indeed, there are few in vivo studies on the effect of silver in the wound healing process. Kjolseth et al. ${ }^{453}$ used a wound healing model in adult mice to study the effect of $\mathrm{AgNO}_{3}$ and SSD on wound epithelialization (regrowing process of skin over a wound) and neovascularization (formation of new blood vessels). They concluded that silver nitrate did not delay the epithelialization and neovascularization in the treated wounds. This is in contradiction with in vitro studies using silver salts ${ }^{423,454}$ in which delays in wound healing, with variations in epithelialization and tissue vascularization, have been observed. It is important to note that certain toxic effects of silver (both on fibroblasts and on microorganisms) are apparently attenuated in vivo by the presence of human serum, other biological fluids containing chloride, peptides, and proteins, ${ }^{455,456}$ or by body fluid dilution. In contrast to silver nitrate and SSD, newly innovated silver-based dressings in which silver itself is incorporated within the dressing rather than being applied as a salt, compound, or solution have been introduced. The most common form of silver found in the silver-based dressings is the so-called nanocrystalline silver, which differs from silver salts. ${ }^{393}$ Nanocrystalline silver products are said to provide small $\operatorname{Ag}(0)$ clusters, which are apparently far less rapidly deactivated by chloride or organic matter than the ionic form. ${ }^{457}$ In comparison to ionic silver, nanocrystalline silver seems to dissolve in water to provide both $\operatorname{Ag}(\mathrm{I})$ and $\operatorname{Ag}(0)$, whereas other silver sources release only $\mathrm{Ag}(\mathrm{I}) .{ }^{393}$ Indeed, such new generation products available on the market have a more controlled and prolonged release of silver to the wound area as compared to cream formulations such as SSD. This mode of silver delivery allows the dressings to be changed less frequently, thereby reducing the risk of nosocomial infections, the cost of care, as well as further tissue damages and patient discomfort. ${ }^{458,459}$ Thus, silver compounds and nanosized silver coatings are examples of very well established and frequently used biomaterials with good antimicrobial and biocompatibility properties. They are used despite increased silver levels in the urine ${ }^{438,460}$ and some (in vitro) studies, which might indicate the contrary. ${ }^{444}$

5.2.2. Bone Contact: Orthopedic and Dental Implants and Bone-Filling Products. Next to our skin, silver can also be in close contact with bone when it is used as a coating for metallic biomaterials or when it is used as AgNPs in bone grafts, ${ }^{461}$ in bone cements, ${ }^{462}$ and with $\mathrm{HA}$ on implant surfaces. ${ }^{463}$ The main applications are here the orthopedic and dental implants. Especially for the latter, where exposure to, for example, bacteria-containing saliva can hardly be avoided, strong antibacterial properties in combination with a good biocompatibility of the implants are required to prevent dramatic consequences such as jawbone infection ${ }^{464}$ and even the loss of denture. Wound infection can however occur as well for all other implants inserted into the body via operations. On the market, metallic alloys, selected for their excellent mechanical properties, resistance to corrosion, and biocompatibility, are available with metallic silver coatings to overcome bacterial infections. They were shown not to provoke any histological change or functional deficit of the organs and no local and systemic side effects despite elevated silver concentrations (see section 4.1). ${ }^{312,313}$ A study ${ }^{465}$ on the dose-dependent effects of silver demonstrated that elementary silver exhibits no cytotoxic effect, at a dose below $143 \mu \mathrm{g} \mathrm{kg}^{-1}$, on osteoblasts in vitro and even stimulates osteogenic maturation. Bone repair itself is a highly concerted process involving osteogenic stem cells, osteoconductive surfaces, and osteoinductive growth factors that can be severely disrupted by bacterial toxins and host inflammatory responses. ${ }^{466}$ Osteointegration (or osseointegration) is thus defined as direct contact between living bone and implant, first described by Branemark and co-workers, ${ }^{467}$ and influenced by various factors such as the design and size of the implant or the surface characteristics of the medical device. ${ }^{468-471}$ Because surgeries and the occurrence of multiresistant bacteria cause significant medical costs, tissueengineered silver bone graft devices have been developed to simultaneously prevent infection while promoting bone regeneration. For instance, Zheng et al. ${ }^{466}$ demonstrated that metallic AgNP-poly(lactic-co-glycolic acid) (PLGA) composite grafts with strong antibacterial properties did not inhibit the adherence, proliferation, or mineralization of MC3T3E1 preosteoblasts, and no negative effects on the bone morphogenetic protein 2 (BMP-2) osteoconductivity were observed. Another group ${ }^{304}$ has investigated the biocompatibility of silver-coated external fixation devices, specifically the interactions of silver with tissue cells. They have shown that silver is neither genotoxic nor cytotoxic and is comparable to stainless steel, a material that is widely used for metal implants. 


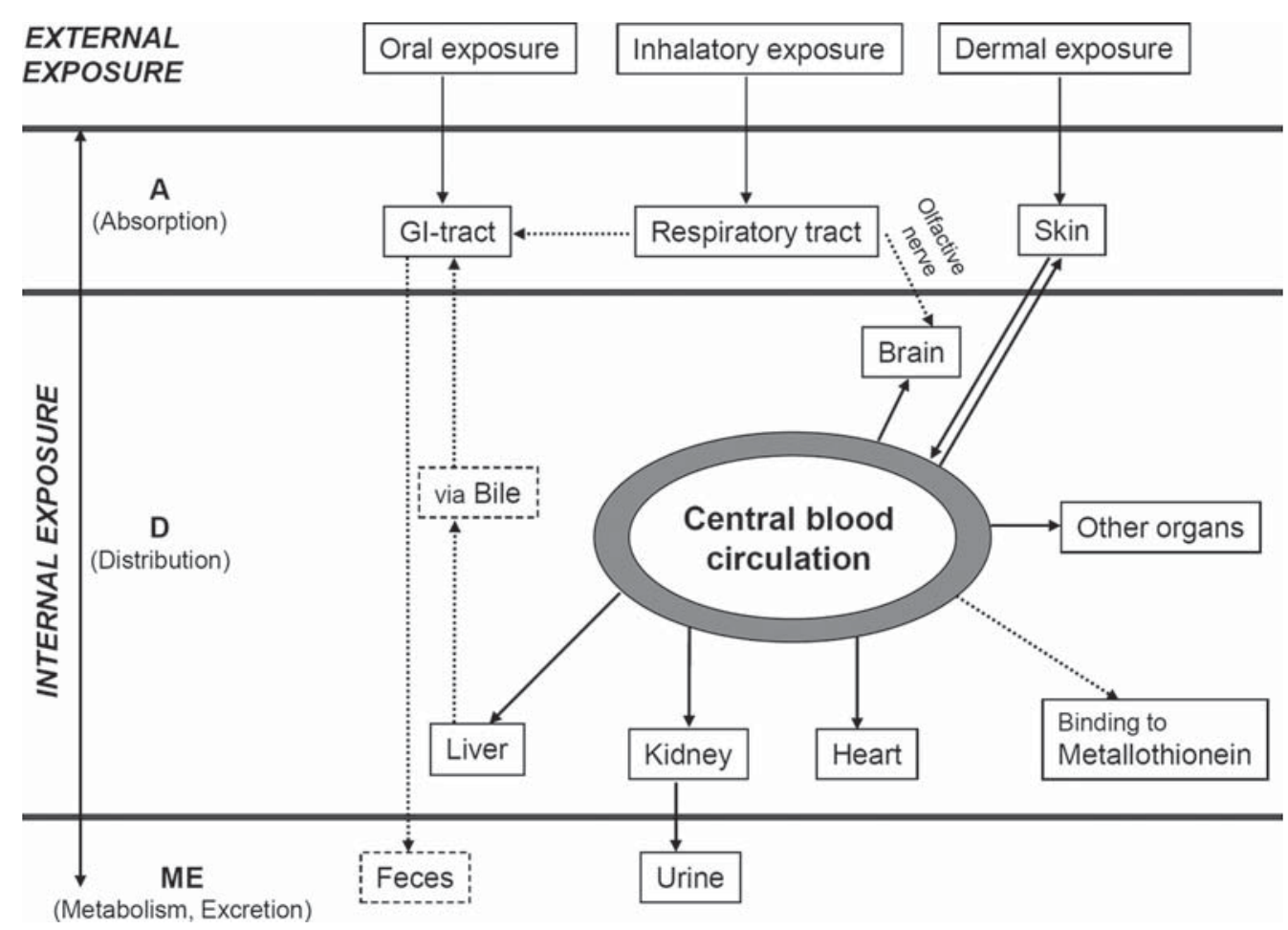

Figure 23. Absorption, distribution, metabolism, and excretion (ADME) processes of nanosilver. Adapted with permission from ref 485 . Copyright 2009 Informa Healthcare.

Moreover, silver coatings caused no cell-cycle delay and revealed good osteoblast activity at long incubation periods. However, the gradual loosening of implants from the bones has been observed, particularly when the implants were cemented. ${ }^{472}$ Yet, in vitro studies of bone cements made of poly(methyl metacrylate) (PMMA) loaded with AgNPs did not display cytotoxicity on human fibroblasts and osteoblasts. ${ }^{352} \mathrm{~A}$ new generation of PMMA/ $\mathrm{Ag}_{2} \mathrm{O}$ bone cements with collagen coating developed by Cavalu and co-workers ${ }^{473,474}$ was shown to be capable of releasing $\operatorname{Ag}(\mathrm{I})$ during a 3-week incubation in a simulated body fluid with ion concentrations approximately equal to those of human blood plasma. With this PMMA/ $\mathrm{Ag}_{2} \mathrm{O}$ cement, biomineralization, biocompatibility, and antibacterial properties were improved.

Although some researchers consider that simply the nanoscale topography of the surface of an implant is sufficient for promoting osteoblast proliferation and therefore bone remineralization, ${ }^{475}$ others have attempted to develop implants with bioactive nanoscale surfaces to promote cell adhesion and bone growth. For example, HA or calcium phosphate coatings contribute to the recalcification of natural bone around the implant thanks to their combination of nanoscale topography and the reservoir of raw material. Such materials, like the cosputtered Ag-containing osteoinductive HA coating on metallic surfaces, ${ }^{353,476}$ developed to accelerate bone attachment in orthopedic implants, show no in vitro cytotoxic effect and promote osteocalcin production (phenotypic marker of the late differentiation state of osteoblast-like cells for osteoinduction and bone mineralization). Therefore, silver addition can apparently stimulate the osteocalcin gene to promote bone mineralization and help the rapid bone in growths in vivo. However, further studies are required to find out the detailed interaction of silver ion with the osteocalcin gene.
5.2.3. Blood Contact: Implants Introduced into the Vascular System. Almost all implants will come at some point into contact with blood. Many polymeric materials such as catheters, vascular grafts, stents, and prosthetic heart valves are even directly introduced into the vascular system and constantly in contact with blood. However, a high number of bloodstream infections and local blood thrombosis have been observed in relation with such implants, and researchers thus started to focus on the development of bioinert and biocompatible coatings for polymer surfaces to minimize nonspecific adhesion and inflammatory events. ${ }^{477}$ PU-based polymers are typical examples. ${ }^{397}$ Polyester-type waterborne PU containing up to $3 \times 10^{4} \mathrm{ppb}\left(3 \times 10^{4} \mu \mathrm{g} \mathrm{kg}^{-1}\right) \mathrm{AgNPs}^{478}$ and PU coated with polyaniline-AgNPs (0.2 wt \%) composite $^{479}$ show good physiological properties in vitro (for fibroblasts, endothelial cells, platelets, and monocytes). Another polymer, PLLA, was shown to remain biocompatible in vivo with a coating containing up to 5 wt $\% \mathrm{AgNO}_{3}\left(5 \times 10^{7} \mu \mathrm{g}\right.$ $\left.\mathrm{kg}^{-1}\right) .{ }^{480}$ Recently, Qureshi et al. ${ }^{358}$ confirmed that the AgNPPLLA nanocomposite films with a AgNP concentration of $7 \times 10^{5} \mathrm{ppb}\left(7 \times 10^{5} \mu \mathrm{g} \mathrm{kg}^{-1}\right)$ had little effect on the viability of human HeLa cells (see also section 4.1.3). In addition, silvercoated polypropylene mesh used in a rat abdominal model ${ }^{481}$ has shown in vitro and in vivo combined antibacterial efficacy with a good biocompatibility as well as tissue integration.

From all of these implants treated with silver and its compounds discussed in this section, silver can in principle be released and, via the bloodstream, reach all organs in the human body, which will be discussed in the following subsection.

\subsection{Effects of Silver Exposure on Tissues and Organs}

With the increasing use of silver, the question of its toxicity becomes more and more an issue as so many sometimes contradictory studies are published. Surprisingly, silver is better tolerated by the body than most heavy metals, ${ }^{482}$ and it is 
relatively nontoxic to mammalian cells. Most humans are exposed every day to very low levels of silver mainly from food and drinking water, but also to a smaller extent from air $\left(1 \times 10^{-6} \mu \mathrm{g} \mathrm{L}^{-1}\right) .{ }^{436}$ The average concentration of silver is $0.2-2.0 \mathrm{ppb}\left(0.2-2 \mu \mathrm{g} \mathrm{L}^{-1}\right)$ in surface water of lakes and rivers, and $200-300 \mathrm{ppb}\left(200-300 \mu \mathrm{g} \mathrm{kg}^{-1}\right)$ in soils. ${ }^{483}$ Silver poisoning, however, only occurs among people chronically exposed to silver. As more and more nanomaterials are produced, some of these materials do exhibit significant toxicity to mammalian cells even if they are biochemically inert and biocompatible in bulk. ${ }^{484}$ Therefore, the exposure to nanosilver in the body is becoming more significant, and silver in the form of nanoparticles has gained an increasing access to tissues, cells, and biological molecules within the human body as shown, for example, in sections 5.1 and 5.2. Once silver compounds are absorbed by the body, three processes are possible, (i) metabolism, (ii) storage, and (iii) excretion, which can result in acute or chronic effects including tissue and organ damages as will be discussed in the following.

5.3.1. Acute and Chronic Toxicity of Silver. Toxic substances, with regard to human health, cause short-term or/ and long-term effects based on their fate in the human body as described by the administration/distribution/metabolism/excretion process $(\mathrm{ADME})^{485}$ depicted in Figure 23. Short-term or acute effects have a relatively quick fate (usually minutes to days) after a brief exposure to a relatively high concentration of toxic material (acute exposure), and they can be local or systemic. Upon close contact with skin, eyes, lungs, or gastrointestinal tract, local effects of the toxicant can be observed rapidly, whereas systemic effects are observed later after absorption of the toxicant into the body from its initial contact point, after transport to other body parts, causing possible adverse effects in susceptible organs.

In the literature, only few studies ${ }^{486}$ describe in vivo results concerning the cytotoxicity of silver. Some report a chronic inflammatory reaction in patients treated with a silver-coated heart valve even though the silver blood concentration did not exceed $22 \mathrm{ppb}\left(22 \mu \mathrm{g} \mathrm{L}{ }^{-1}\right),{ }^{420}$ while other studies describe no cytotoxic effect of silver and a good biocompatibility. ${ }^{276}$ For instance, for clean wounds in pigs, SSD was shown to increase the epithelialization rate by $28 \%$, indicating a beneficial effect in wounds additional to antimicrobial activity. ${ }^{487}$ These contradictory results can be explained by the variation of the silver concentration acting on different cell types. It is well-known that silver toxicity is a dose-dependent process. The estimated fatal dose of $\mathrm{AgNO}_{3}$ is $1.4 \times 10^{5} \mu \mathrm{g} \mathrm{kg}^{-1}$ for humans. ${ }^{488}$ Most of the quantitative safety data on silver come, however, from a large number of animal studies done in the past century. Thus, the oral lethal dose, $50 \%\left(\mathrm{LD}_{50}\right)$, which is the amount of the substance required, usually per body weight, to kill $50 \%$ of the test population reported for $\mathrm{AgNO}_{3}$ is $0.5-1.29 \times 10^{5} \mu \mathrm{g} \mathrm{kg}^{-1}$ for mice, depending on the references, ${ }^{489}$ and an $\mathrm{LD}_{50}$ of $2.8 \times 10^{6} \mu \mathrm{g} \mathrm{kg}^{-1}$ for rats $\left(1.0 \times 10^{6} \mu \mathrm{g} \mathrm{kg}^{-1}\right.$ for mice $)$ is given for the relatively insoluble silver oxide. ${ }^{490}$ Female SJL $\left(\mathrm{H}-2^{\mathrm{s}}\right)$ mice (Swiss mouse inbred by James Lambert) developed serum IgG antinucleolar antibodies after a 5-10 week treatment with $0.05 \%\left(5 \times 10^{5} \mu \mathrm{g} \mathrm{L}^{-1}\right)$ or $0.01 \%\left(1 \times 10^{5} \mu \mathrm{g} \mathrm{L}^{-1}\right) \mathrm{AgNO}_{3}$, administered orally in drinking water. ${ }^{491}$ Controls receiving ordinary tap water and mice treated with $0.002 \%$ $\left(2 \times 10^{4} \mu \mathrm{g} \mathrm{L}^{-1}\right) \mathrm{AgNO}_{3}$ showed no antinucleolar antibodies. Serum autoantibodies to chromatin or histones, kidney, spleen, stomach, thyroid, or skin antigens (except the nucleolus) were not found in any of the mice. In mice given $0.05 \%$ $\left(5 \times 10^{5} \mu \mathrm{g} \mathrm{L}^{-1}\right) \mathrm{AgNO}_{3}$, the kidney showed the highest silver concentration $\left((1.22 \pm 0.9) \times 10^{4} \mu \mathrm{g} \mathrm{kg}^{-1}\right.$ wet wt $)$, followed by the spleen $\left((8.7 \pm 1.3) \times 10^{3} \mu \mathrm{g} \mathrm{kg}^{-1}\right.$ wet wt $)$, and the liver $\left((3.9 \pm 0.4) \times 10^{3} \mu \mathrm{g} \mathrm{kg}^{-1}\right.$ wet wt $)$. Lower concentrations of $0.01 \%\left(1 \times 10^{5} \mu \mathrm{g} \mathrm{L}^{-1}\right) \mathrm{AgNO}_{3}$ caused a different distribution of silver, with the highest concentration now in the spleen $\left((2.1 \pm 0.16) \times 10^{3} \mu \mathrm{g} \mathrm{kg}^{-1}\right.$ wet $\left.\mathrm{wt}\right)$, followed by the kidney $\left((6.3 \pm 0.37) \times 10^{2} \mu \mathrm{g} \mathrm{kg}^{-1}\right.$ wet $\left.\mathrm{wt}\right)$ and the liver $\left(<(0-29) \times 10^{3} \mu \mathrm{g} \mathrm{kg}^{-1}\right.$ wet wt $)$. Silver seems thus to be a more specific inducer of antinucleolar/ antifibrillarin autoantibodies than mercury and gold, lacks the general immune stimulating potential of mercury, and has only a weak tendency to induce renal immune deposits. ${ }^{491}$

Chronic toxicity of silver is observed with argyria, which was observed in individuals that have ingested both metallic silver and silver compounds in small doses over months. To induce argyria by ingestion, the estimated required amount lies within the range of $(1.4-40) \times 10^{4} \mu \mathrm{g} \mathrm{kg}^{-1}$ for soluble silver salts. ${ }^{488,492}$ Argyria is permanent, and most medical doctors and scientists believe that the discoloration of the skin seen in argyria is the most serious health effect that silver may provoke. ${ }^{483}$ While the silver level in the blood in argyremic patients is reported to vary from nondetectable to $5 \mu \mathrm{g} \mathrm{L}^{-1}$, levels below $2 \mu \mathrm{g} \mathrm{L}^{-1}$ in the blood are considered as normal ${ }^{460}$ based on daily incorporation of small amounts of silver. Indeed, only a fraction of it (almost $10 \mathrm{wt} \%$ ) is absorbed, ${ }^{494}$ the rest being excreted in feces. ${ }^{438,494}$ The absorbed fraction is carried by the bloodstream and deposited in various tissues throughout the body in which silver is stored and accumulated intracellularly apparently without any effect. ${ }^{494}$ Most of the ingested silver is then excreted via the urine. ${ }^{438,494}$

However, therapeutic and toxic effects can be only exhibited by free $\mathrm{Ag}(\mathrm{I})$ (see section 4). Therefore, the reported threshold values have to be interpreted with caution, because the measured silver ion concentration may include both bound and free silver. In the literature, the cytotoxicity of silver nitrate on mammalian cells has been reported: the half maximal inhibitory concentration $\left(\mathrm{IC}_{50}\right)$, which described the effectiveness of a compound in inhibiting biological or biochemical function, is $721 \mathrm{ppb}\left(721 \mu \mathrm{g} \mathrm{L}^{-1}\right)$ for L929 mouse fibroblasts and $470 \mathrm{ppb}\left(470 \mu \mathrm{g} \mathrm{L}^{-1}\right)$ for MC3T3-E1 mouse osteoblasts after 8 days of incubation in Eagle's minimal essential medium (MEM). ${ }^{495}$

Apart from these more general toxicity aspects, the effects of silver on individual organs have been studied, which will be discussed in more detail in the next subsection.

5.3.2. Tissue and Organ Damages. After an overexposure followed by absorption, silver is transported by the bloodstream and can accumulate in organs and tissues such as liver, skin, kidney, spleen, heart, lung, olfactory bulb, corneas, gingival mucous membranes, brain, and testes. ${ }^{438,496-502,502,503}$ Several systemic clinical side effects of silver may occur, such as argyrosis (eye), leucopenia, or liver and kidney damage. ${ }^{420,430,496,504}$ For instance, histopathological analysis of liver tissue showed a dose-dependent toxicity of silver ${ }^{44,505}$ and an inflammation with an influx of lymphocytes, but values can recover after aborting silver uptake. ${ }^{438}$

Reports concerning the toxic effects of silver related to nerve tissue, centrally and in the periphery, are rather rare. However, silver has been claimed to cause brain damage, ${ }^{506}$ seizure, ${ }^{507}$ a persistent vegetative state, ${ }^{508}$ or in early studies even the death of the animal. ${ }^{509,510}$ Moreover, Horner et al. ${ }^{511}$ intoxicated mice with an intraperitoneal injection of $\mathrm{LD}_{50}$ amounts of different 

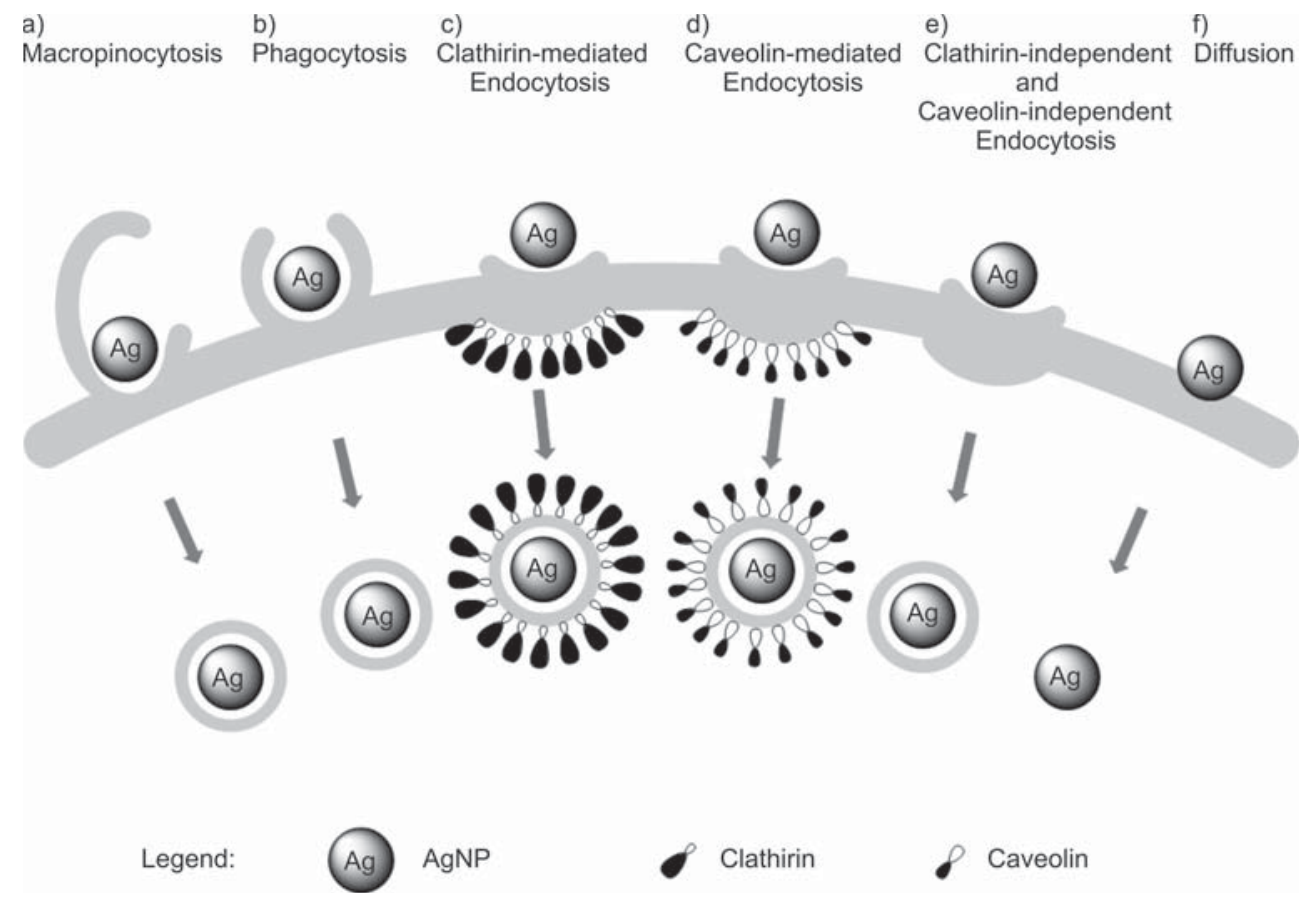

Figure 24. Possible modes of AgNPs internalization. Adapted with permission from ref 519. Copyright 2010 Macmillen Publishers Ltd. Adapted with permission from ref 520. Copyright 2011 Springer Science and Business Media.

silver compounds and observed toxic syndromes including hyperexcitability, ataxia, central nervous system depression, loss of righting reflex, and death of mice. At lower doses, different toxic effects have been reported. For example, local neurotoxicity was reported in a patient 5 years after the use of silverimpregnated bone cement ${ }^{512,513}$ in a revisional Christiansen total hip arthroplasty. After serum analysis, silver-impregnated bone cement was shown to release a high silver concentration in the hip joint cavity leading to the peripheral nerve lesion. If a large amount of silver is released from the implanted bead (only $10 \%$ of the loaded silver remained on the bead surface) into connective tissue, severe local tissue disruption and even necrosis can be induced. ${ }^{514}$ For silver-coated heart valves, hypersensitivity or allergic reactions were described. ${ }^{515,516}$

The severness of tissue damage seems thus to be dose dependent, and low concentration of silver compounds appears to be administrable without lethal side effects.

5.3.3. Cellular Uptake of Silver. As mentioned before, the nanoparticles in general can get into the body through semiopen interfaces (Figure 23). On the cellular level, modes of the internalization include either passive diffusion ${ }^{192,517,518}$ or active endocytic pathways, such as (i) macropinocytosis, (ii) phagocytosis, (iii) clathirin-mediated endocytosis, (iv) caveolarmediated endocytosis, and (v) clathirin-independent and caveolin-independent endocytosis (Figure 24). ${ }^{192,519,520}$ These processes seem to depend on the size and the shape of the nanoparticles. ${ }^{517,519}$ In a study by AshaRani et al., ${ }^{192}$ the presence of endosomes filled with AgNPs, as seen on TEM and scanning TEM images, suggests a receptor-mediated endocytic pathway rather than a diffusion process. One way to elucidate how particles enter the cell is the selective inhibition of the different pathways. For example, treating human macrophage cell line U937 with different inhibitors, such as chlorpromazine, nystatin, and cytochalasin D, and measuring subsequent production of Interleukin-8, Kim and $\mathrm{Choi}^{519}$ proposed a complex mechanism, including endocytosis and phagocytosis, for the uptake of AgNPs. The same authors proposed, in addition, direct penetration, because of the observed cell death. ${ }^{517}$ On the other hand, Greulich et al. ${ }^{521}$ investigated uptake of PVP-coated AgNPs by human mesenchymal stem cells. Using inhibitors, such as chlorpromazine and wortmannin, the main uptake through clathirin-mediated endocytosis and macropinocytosis was indicated, respectively. ${ }^{521}$ For more detailed information about the general mechanism of cellular uptake of NPs, the reader is directed to recently published reviews in this field. ${ }^{519,520,522}$

\subsection{Toxic Effects of Silver Nanoparticles}

Since the recent developments of AgNPs in medical applications (see section 4), their toxic effects have also to be studied. Indeed, AgNPs interact with bacteria and viruses (see section 3) and with the eukaryotic immune system, as will be shown in the following.

Toxic effects of AgNPs have been reported for different in vitro and in vivo mammalian systems. ${ }^{523-528}$ Indeed, AgNPs can potentially cause toxic effects, such as inflammation, cell activation, depletion of GSH level in association with mitochondrial dysfunction, and ROS production. ${ }^{529}$ Indeed, AgNPs interact with thiol-containing proteins and enzymes, such as thioredoxin peroxidase, thioredoxin, superoxide dismutase protein, and GSH (the most sensitive indicator of the cell to silver toxicity). The so-induced ROS accumulation can initiate an inflammatory response, a perturbation, and a destruction of the mitochondria. ${ }^{530}$ Arora et al. ${ }^{531}$ confirmed in vitro an induction of oxidative stress with a depletion of GSH and an increased lipid peroxidation when human cell lines were exposed to AgNPs. Finally, all of these processes gradually lead to necrosis and/or programmed cell death (apoptosis). ${ }^{532,533} \mathrm{It}$ has been found that apoptosis was induced by a lower silver concentration than the one required for necrosis. ${ }^{531}$ However, how exactly AgNPs do induce cytotoxicity is still not well understood. Lubick et al. ${ }^{534}$ suggested that AgNPs can be taken up by organisms, thereby introducing a continuous release of 


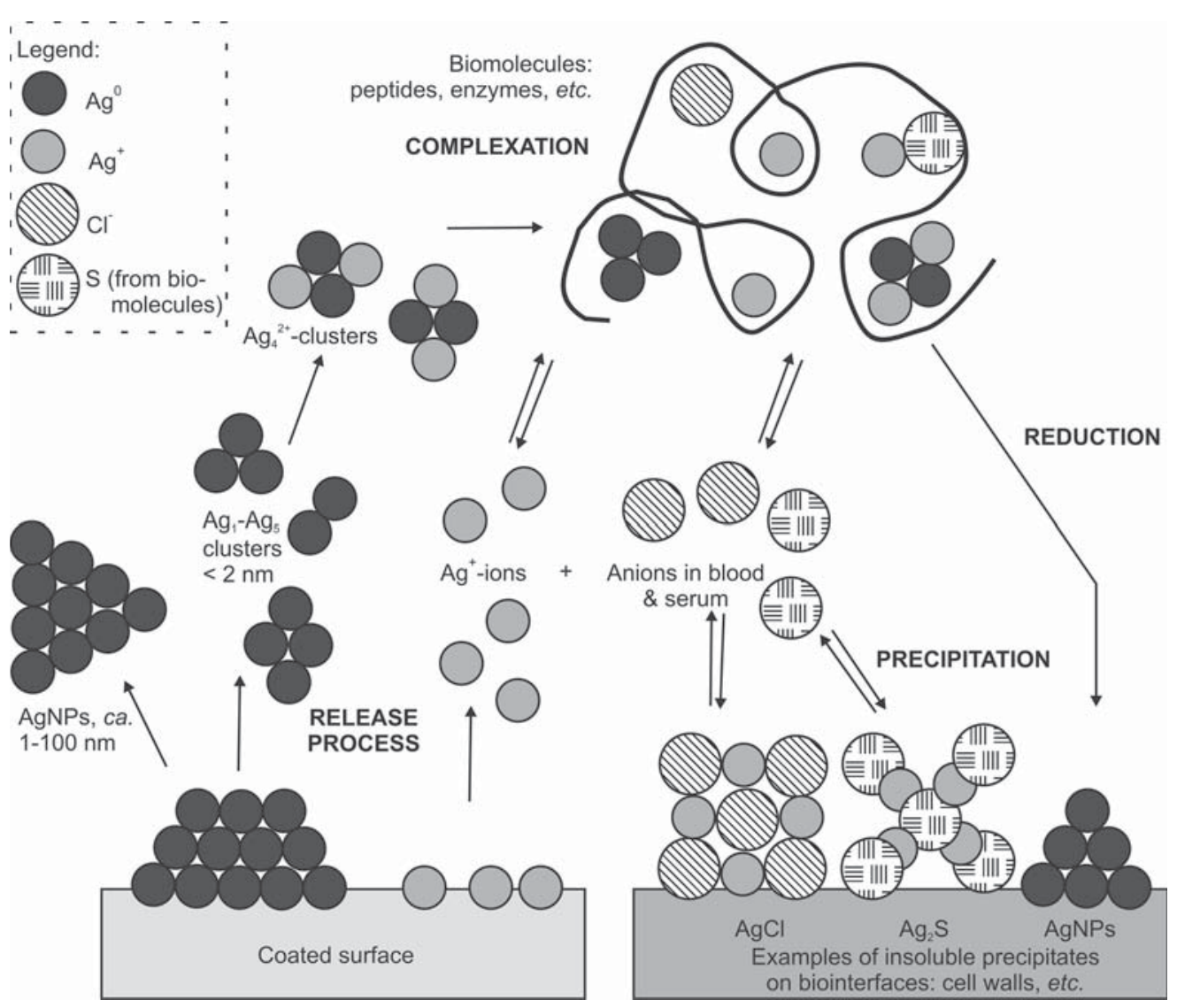

Figure 25. Overview on currently discussed species of $\operatorname{Ag}(\mathrm{I})$ and $\mathrm{Ag}(0)$ and their interactions with biomolecules starting with their release from surfaces such as catheters or implants $\left(K_{\mathrm{SP}}\left(25^{\circ} \mathrm{C}\right): \mathrm{Ag}_{2} \mathrm{~S}=6 \times 10^{-51} \mathrm{~mol}^{3} \mathrm{~L}^{-3}, \mathrm{AgCl}=1.8 \times 10^{-10} \mathrm{~mol}^{2} \mathrm{~L}^{-2}, \mathrm{Ag}_{3} \mathrm{PO}_{4}=8.89 \times 10^{-17} \mathrm{~mol}^{4} \mathrm{~L}^{-4}\right)$. ${ }^{194}$

silver ions into the tissues by bypassing the mechanism of silver ion detoxification, discussed in section 5.4. As was already mentioned in the context of bacteria (see section 3.1), it has recently been suggested that AgNPs may act as Trojan horses by entering the cells and then releasing silver ions that damage intracellular functions. ${ }^{527}$ Recent publications reviewed the human risk assessment for nanoforms of silver (e.g., AgNPs $)^{498}$ and the toxicology and toxicokinetics of nanosilver. ${ }^{535}$ One should keep in mind that the main in vitro mechanism seems to be mediated by an increase in ROS production. This stimulates oxidant-sensitive signaling pathways, which at high doses stimulate inflammation, leading to a secondary genotoxicity and cell death (apoptosis or necrosis).

Smaller particles exhibit not only a higher toxicity as compared to larger particles due to their larger surface area availability and thus a stronger ion release (as explained in section 3.1), they can in addition cross more easily the biological barriers, such as the blood-brain ${ }^{536,537}$ or the blood-testes barrier. ${ }^{537,538}$

\subsection{Silver Detoxification}

Although the antimicrobial activity of silver is well-known, little is known about the eukaryotes' detoxification mechanisms. The question is often raised why silver does not have similar cytotoxic effects toward eukaryotic cells compared to bacterial cells. Eukaryotic cells are usually larger with a higher structural and functional redundancy as compared to prokaryotic cells, and therefore higher silver ion concentrations are required to achieve comparable toxic effects as for bacterial cells. ${ }^{27}$ This difference provides a "therapeutic window" (see section 4) in which bacterial cells are successfully attacked, whereas harmful effects on eukaryotic cells are not yet observed. From the literature, several modes of silver detoxification exist in eukaryotic cells: (i) the sequestration of silver ions and (ii) the ATPase efflux pump.

5.5.1. Sequestration of Silver Ions. The principal sequestration agents for transition metal ions in groups 11 and 12 , including $\mathrm{Cu}(\mathrm{I})$ and $\mathrm{Ag}(\mathrm{I})$, are sulfur-containing molecules such as sulfides ${ }^{494}$ or the cysteine-rich metallothioneins (see also section 2.2). ${ }^{539}$ One of the first observable interactions of silver salts in contact with the skin are black, insoluble precipitates discussed as sulfidic compounds. ${ }^{540}$ Surprisingly, their exact composition is still not known. However, many organisms use sulfides to precipitate the excess of metals. The precipitation of silver ions (Figure 25) in the form of silver sulfide, but also as silver phosphate or silver chloride, renders $\mathrm{Ag}(\mathrm{I})$ less bioavailable due to the low solubility of these compounds $\left(K_{\mathrm{SP}}\left(25^{\circ} \mathrm{C}\right)\right.$ : $\mathrm{Ag}_{2} \mathrm{~S}=6 \times 10^{-51} \mathrm{~mol}^{3} \mathrm{~L}^{-3}, \mathrm{AgCl}=1.8 \times 10^{-10} \mathrm{~mol}^{2} \mathrm{~L}^{-2}$, $\left.\mathrm{Ag}_{3} \mathrm{PO}_{4}=8.89 \times 10^{-17} \mathrm{~mol}^{4} \mathrm{~L}^{-4}\right) .{ }^{194}$ However, the nature of the solutes can affect the biological activity of silver, for example, by complex formation, thus influencing the amount of silver in solution. In, for example, phosphate-buffered saline (PBS), silver can be active in concentrations as low as $50 \mathrm{ppb}$ $\left(50 \mu \mathrm{g} \mathrm{L}^{-1}\right)$, but the presence of organic matter significantly diminishes the efficiency of silver. Nutrient broths decrease the efficiency of silver by a factor of at least 80 as compared to pure water, ${ }^{541}$ and serum decreases the activity by a factor of more than $250 .{ }^{542}$ Serums are an extremely complex mixture of plasma proteins, growth factors, hormones, etc. The exact mechanism involved in serum-promoted silver release is still 
unknown, but the presence of sodium and chloride ions has an effect on silver dissolution. Thus, Burd et al. ${ }^{543}$ showed that when the dressing was presoaked in PBS, the release of silver was increased, leading to a decrease of the silver concentrations remaining on the dressing, and the in vitro toxicity of silver in both keratinocyte or fibroblast culture media was lowered. Indeed, Hidalgo and co-workers ${ }^{444}$ also demonstrated that viability inhibition of fibroblasts varies according to the concentrations of fetal calf serum (FCS) present in the medium. $\mathrm{AgNO}_{3}$ was 10 times less toxic in a medium supplemented with 10 wt \% FCS than with 2-5\% FCS. These results suggested that FCS in the culture media inactivates $\mathrm{AgNO}_{3}$ or protects the fibroblasts against the toxic effects of this compound. Such cytoprotective effects of serums have also been described and confirmed by other researchers, ${ }^{544,545}$ however, without a full description of a molecular mechanism.

The previously mentioned argyria (see section 5.3.1) is an effect resulting from Ag-sequestration in the body. Such pigmentation caused by silver ingestion, as well as tattoos (pigmented lesions caused by amalgam), are examples of silver remaining in the body for an extended period without causing other harm. ${ }^{546}$ In the case of argyria, White et al. ${ }^{549}$ demonstrated the presence of silver, sulfur, and selenium in the basement membrane and elastic fibers. Aoyagi and Katagiri $^{546}$ also reported the presence of silver and sulfur in connective tissues without host reaction. Using an original subcutaneous injection method, Aoyagi and Iwasaki ${ }^{548}$ have implanted silver particles of two different sizes $(100 \mathrm{~nm}$ and $45 \mu \mathrm{m})$ and have observed the sulfuration of silver and histopathologic changes during a year. From the obtained results, they concluded that the decline of silver effect on the host was correlated with an increase in the sulfuration of silver. The storage of silver in form of $\mathrm{Ag}_{2} \mathrm{~S}$ or S-bound species in general seems thus apparently an efficient way of detoxification. Another prominent example for the importance of sulfurcontaining compounds are the metallothioneins already mentioned in section 2.2. Metalloproteins in general protect the human body against oxidative stress induced by excess free radicals. The synthesis of MT is transcriptionally regulated by metals in all higher eukaryotes, ${ }^{539}$ and its production promotes RNA and DNA synthesis, cell proliferation, and tissue repair. Thus, MTs found in animals, higher plants, eukaryotic microorganisms, and some prokaryotes ${ }^{539}$ have the dual effect of protecting the tissues against the potential toxic effects of silver and of improving natural healing. Indeed, the uptake of silver ions by the cells stimulates MT synthesis and leads to the formation of stable complexes, such as Ag-MT with luminescent properties. ${ }^{549}$ The metallothioneins are still subjects of scientific investigations due to their vital roles in mineral transport, enzyme production, signal transduction, redox reactions, and many other crucial biological functions.

In summary, silver is thought to be bonded to the host substances mainly through the coordination by sulfur, thus leading to a reduced toxicity of the metal ion.

5.5.2. Silver Ion Transport by ATPase P-type Efflux Pumps. We have discussed in section 3.2 that the bacterial resistance of silver consists of two mechanisms: (i) the accumulation and storage of silver (generally redox chemistry) and (ii) the active efflux pump, in which conserved regions of P-type ATPase associated with the transport of copper pump the silver ions out of the cell. ATPase pumps are ubiquitous and highly conserved from bacteria to humans. ${ }^{550-552}$ In mamma- lian cells, they are present in the plasma membranes or endoplasmic reticular membranes, whereas in prokaryotes they are located in the cytoplasmic membranes. ATPases, which translocate ions such as protons, $\mathrm{Na}(\mathrm{I}), \mathrm{K}(\mathrm{I}), \mathrm{Ca}(\mathrm{II})$, and a variety of metal ions, are categorized into the P-type family of ATPases ${ }^{553}$ that use the energy provided by the ATP hydrolysis to transport ions across cell membranes. In eukaryotes, P-type ATPases play essential roles in numerous processes, which in humans include nerve impulse propagation, relaxation of muscle fibers, secretion and absorption in the kidney, acidification of the stomach, and nutrient absorption in the intestine. $^{554}$

Similar to bacterial cells, silver has been shown to use the copper transporter in eukaryotic systems, such as ATP7A that transports the intracellular silver in copper-resistant Chinese hamster ovary cells (CHO-CUR3) and in human fibroblasts to the plasma membrane for excretion. ${ }^{555,556}$ ATP7A and the related ATP7B are well characterized as copper transporters with distinct distribution in tissues. Both play an equally important role in regulating copper levels in the body and in maintaining intracellular copper concentration. ${ }^{557}$ These two transporters utilize the same general mechanism for their function, and are coexpressed in some cells, but they have different trafficking properties. While ATP7B is more specialized, ATP7A has been found to be a "house-keeping" enzyme due to its higher turnover rate for the formation of phosphorylated intermediates ${ }^{558}$ as compared to ATP7B. ATP7A exports copper from the enterocytes (or intestinal absorptive cells) into the blood circulation for further distribution throughout the body. ${ }^{559}$ Inactivation of ATP7A in Menkes Disease results in increased copper concentration in the intestine. ${ }^{560}$ Removal of excess copper from the body requires the presence of ATP7B in the liver. ${ }^{561}$ ATP7B facilitates transport of copper from hepatocytes into the bile. ${ }^{562}$ Thus, the inactivation of ATP7B in Wilson's disease is associated with a massive copper overload in the liver and some other tissues. ${ }^{560}$ Silver transport by these transporters is expected to take place in an analogous fashion.

Another monitored protein, CTR1, has an important role in regulating the $\mathrm{Cu}(\mathrm{I})$ uptake at the cell membrane in mammalian cells by transporting copper with high affinity in a time-dependent and saturable manner. ${ }^{563}$ This protein can similarly handle silver and other metals. ${ }^{563-565}$ However, little is known about the expression and function of CTR1 for either silver or copper transport. ${ }^{566}$ Because of the aforementioned close similarity between $\mathrm{Cu}(\mathrm{I})$ and $\mathrm{Ag}(\mathrm{I})$ (see, for example, sections 2.1 and 2.2), it is not surprising that silver ions were found to interact with important copper transport proteins such as ATP7 and CTR1.

To conclude, AgNPs and all implants treated with silver and its compounds release $\operatorname{Ag}(\mathrm{I})$, which are first absorbed by the body and then transported via the bloodstream to be accumulated mainly in the liver and spleen, causing some side effects in a dose-dependent manner. For the time being, little is known about the mechanisms involved in the silver transport and detoxification in the human body. Because silver is a nonessential element for our human body, $\operatorname{Ag}(\mathrm{I})$ has to use already existing transport ways for other, essential elements. Analogies can be made (i) on the basis of the similarity of ATP pumps ranging from bacteria to humans, and (ii) by direct comparison with $\mathrm{Cu}(\mathrm{I})$ transport due to the comparable properties of $\mathrm{Cu}(\mathrm{I})$ and $\mathrm{Ag}(\mathrm{I})$ (see, for example, sections 2.1 and 2.2). Thus, according to numerous predictions, silver may 
use the already existing Cu-transport systems. Nevertheless, the real pathway still remains to be elucidated.

The determination of a "therapeutic window" and the toxicity or the biocompatibility of silver nanomaterials and silver-coated implants is crucial for their successful application. Nonetheless, this is also one of the main difficulties because some in vivo and in vitro studies give contradictory results depending on the used models and conditions.

\section{CONFLICTING EVIDENCE}

This Review on the current state of the art of research on silver and its compounds in the medical context shows that most results need to be interpreted with care and that further investigations are needed. For instance, wound care studies, trying to compare different products, have been carried out by several research groups, but varying silver concentrations and differing modes of delivery of silver ions (test setup) make a direct comparison inappropriate. ${ }^{439,567-570}$ Another example showing the difficulties in the interpretation of the obtained results lies in the in vitro antimicrobial tests. For instance, laboratory studies usually begin with the evaluation of the determination of the MIC of an antimicrobial agent to determine its potency. Numerous factors, however, influence the activity, such as the concentration, the temperature, the contact time, the type of tested species, the number of organisms present, and the presence of organic matter. ${ }^{221,571}$ This is why such MIC-values may vary from one literature to another. Furthermore, recent studies of 3D cell cultures show that, for example, skin cells are better able to survive exposure to cytotoxic agents such as silver nitrate than in $2 \mathrm{D}$ cultures. ${ }^{572}$ Because 3D cell cultures have only recently become more standardized for investigations, the literature currently lacks more abundant results. Along the same lines, we have also pointed out the often contradictory results obtained for in vivo as compared to in vitro studies (see sections 3 and 5). In this context, equilibria of insoluble silver compounds with soluble biomolecule complexes are often cited, but the nature of the latter remains largely unknown, and as insoluble silver compounds, $\mathrm{AgCl}$ is as often mentioned as $\mathrm{Ag}_{3} \mathrm{PO}_{4}$ or $\mathrm{Ag}_{2} \mathrm{~S}$, although the solubility products of these are very different.

Another ongoing discussion is the question whether bacteria can become resistant to silver. We have described indeed several defense mechanisms of bacteria against silver: the efflux pumps and silver reducing mechanisms. A recent review ${ }^{573}$ describes thus that bacteria and fungi are able to biosynthesize AgNPs of 5-40 nm from relatively high concentrated $\mathrm{AgNO}_{3}$ solutions $\left(1 \times 10^{3} \mu \mathrm{M}, 1.08 \times 10^{5} \mu \mathrm{g} \mathrm{L}^{-1}\right){ }^{183}$ If such properties are encoded in genes, they are very likely to be transmissible, either by heritage or by horizontal transfer. However, given the wide range of possible interactions of silver ions with biomolecules, chances of absolute resistance against silver as for antibiotics are probably very much smaller. The main concern for applicability will thus be to remain in the "therapeutic window".

Studying the literature along this Review, we also discovered discrepancies in the description of AgNPs, "nano-silver", and silver clusters in different papers mentioned in this Review. It is not clear if the same or different species are described, for example, for clusters formulated as " $\mathrm{Ag}_{4}$ " and " $\mathrm{Ag}_{4}{ }^{2+}$ ", as the analytical methods to analyze these clusters were different. Mass spectrometry certainly would reveal rather the charged species, and at the same time may lead to fractionation of clusters, thus falsifying the results. XPS studies, on the other hand, may give better insights into the oxidation state of the metal directly in the tissues. This method is however less used by biochemists and microbiologists. The term "silver nanoparticles", AgNPs, is also widely used and may describe very different sizes of NPs, on one hand, as well as very different surface chemistry of the latter. Thus, some studies come to the conclusions that AgNPs are rather toxic, while others describe them as well tolerable by the mammalian organism. Certainly, the size of the AgNPs plays just as important of a role as its surface functionalization. Their surface charge is important if these particles should be taken up easily or not, if they are hydrophobic or hydrophilic, and thus soluble rather in apolar, respectively polar, environments.

\section{CONCLUSIONS}

We have brought together in the previous sections the results related to the interaction of silver and its compounds with living cells, giving an integrating view from the materials via the microscopic to the molecular scale. While on the materials side, a lot of facts are confirmed, the antimicrobial properties observed at the nano- and microscale are more difficult to fit to the molecular observations. The way from hypotheses to a full molecular mechanism of action of the silver ions in biological media is still long, and a lot of bridges need to be built between the research areas. While some results and opinions seem to be diametrically opposite, they often are based on experiments, which cannot be compared directly with each other. It is established by now that the interactions of silver and its ions with human and bacterial cells take place on many molecular levels. Thus, the current state of research on silver and its compounds in medicine resembles a very large puzzle of which partial areas give a local picture on the mechanism, while other areas are still mainly incomplete. A number of different hypotheses, sometimes of opposite conclusions, are derived from individual results to propose the missing links. However, these have to be confirmed yet to complete the "silver puzzle" and to understand the overall reactions and underlying mechanisms of its action.

We have seen in these past sections that, although many results on silver and its interactions with biological media such as body tissues and fluids as well as individual biomolecules can be found in the literature, a global picture on the functioning is still missing. Many contradictory studies confirm that the story about silver and its good antimicrobial properties and good biocompatibility is by far not finished. Silver definitely has a great potential in the medical context as long as its concentration can be well controlled. With further studies, the potential for applications in other fields such as nanomaterials is extremely high and promising.

It is thus important to foster interdisciplinary research, regrouping scientists from all domains of natural sciences to solve a mature problem: we have to combat bacteria, which become more and more resistant against antibiotics. Silver and its compounds may well be a possible solution.

\section{AUTHOR INFORMATION}

\section{Corresponding Author}

*Phone: +41263008732. Fax: +41263009738. E-mail: sonja. eckhardt@unifr.ch (S.E.); katharina.fromm@unifr.ch (K.M.F.).

\section{Notes}

The authors declare no competing financial interest. 
(3) Leid, J. G.; Ditto, A. J.; Knapp, A.; Shah, P. N.; Wright, B. D.; Blust, R.; Christensen, L.; Clemons, C. B.; Wilber, J. P.; Young, G. W.; Kang, A. G.; Panzner, M. J.; Cannon, C. L.; Yun, Y. H.; Youngs, W. J.; Seckinger, N. M.; Cope, E. K. J. Antimicrob. Chemother. 2011, 67, 138.

(4) Youngs, W. J.; Knapp, A. R.; Wagers, P. O.; Tessier, C. A. Dalton Trans. 2012, 41, 327.

(5) Roy, M.; Fielding, G. A.; Beyenal, H.; Bandyopadhyay, A.; Bose, S. ACS Appl. Mater. Interfaces 2012, 4, 1341.

(6) Ragaseema, V. M.; Unnikrishnan, S.; Kalliyana Krishnan, V.; Krishnan, L. K. Biomaterials 2012, 33, 3083.

(7) Furno, F. J. Antimicrob. Chemother. 2004, 54, 1019.

(8) Shimazaki, T.; Miyamoto, H.; Ando, Y.; Noda, I.; Yonekura, Y.; Kawano, S.; Miyazaki, M.; Mawatari, M.; Hotokebuchi, T. J. Biomed. Mater. Res. 2009, 92B, 386.

(9) Kraft, C. N.; Hansis, M.; Arens, S.; Menger, M. D.; Vollmar, B. J. Biomed. Mater. Res. 2000, 49, 192.

(10) Silver, S. FEMS Microbiol. Rev. 2003, 27, 341.

(11) Silver, S.; Le Phung, T.; Silver, G. J. Ind. Microbiol. Biotechnol. 2006, 33, 627 .

(12) Hill, J. W. Colloidal Silver; Clear Springs Press: Rainier, 2009.

(13) Robin, A. Y.; Fromm, K. M. Coord. Chem. Rev. 2006, 250, 2127.

(14) Silver, F. H.; Christiansen, D. L. Biomaterials Science and Biocompatability; Springer: New York, 1999.

(15) Yildirimer, L.; Thanh, N. T.; Loizidou, M.; Seifalian, A. M. Nano Today 2011, 6, 585.

(16) Blecher, K.; Nasir, A.; Friedman, A. Virulence 2011, 2, 395.

(17) Bhattacharyya, S.; Kudgus, R. A.; Bhattacharya, R.; Mukherjee, P. Pharm. Res. 2011, 28, 237.

(18) Che, C.-M.; Siu, F.-M. Curr. Opin. Chem. Biol. 2010, 14, 255.

(19) Waldron, K. J.; Robinson, N. J. Nat. Rev. Microbiol. 2009, 7, 25.

(20) Pearson, R. G. J. Am. Chem. Soc. 1963, 85, 3533.

(21) Letelier, M. E.; Faúndez, M.; Jara-Sandoval, J.; Molina-Berríos, A.; Cortés-Troncoso, J.; Aracena-Parks, P.; Marín-Catalán, R. J. Appl. Toxicol. 2009, 29, 695.

(22) Shoeib, T.; Siu, K. W. M.; Hopkinson, A. C. J. Phys. Chem. A 2002, 106, 6121.

(23) Jover, J.; Bosque, R.; Sales, J. Dalton Trans. 2008, 45, 6441.

(24) Jover, J.; Bosque, R.; Sales, J. J. Phys. Chem. A 2009, 113, 3703.

(25) Glusker, J. P. Adv. Protein Chem. 1991, 42, 1.

(26) Shoeib, T.; Rodriquez, C. F.; Siu, K. W. M.; Hopkinson, A. C. Phys. Chem. Chem. Phys. 2001, 3, 853.

(27) Romanov, V.; Siu, C.-K.; Verkerk, U. H.; Aribi, H. E.; Hopkinson, A. C.; Siu, K. W. M. J. Phys. Chem. A 2008, 112, 10912.

(28) Lee, V. W.-M.; Li, H.; Lau, T.-C.; Guevremont, R.; Siu, K. W. M. J. Am. Soc. Mass Spectrom. 1998, 9, 760.

(29) Cooks, R. G.; Wong, P. S. H. Acc. Chem. Res. 1998, 31, 379.

(30) Yamauchi, O.; Odani, A.; Takani, M. J. Chem. Soc., Dalton Trans. 2002, 18, 3411.

(31) Acland, C. B.; Freeman, H. C. J. Chem. Soc. D 1971, 17, 1016.

(32) Kamwaya, M. E.; Papavinasam, E.; Teoh, S. G.; Rajaram, R. K. Acta Crystallogr., Sect. C: Cryst. Struct. Commun. 1984, 40, 1318

(33) Kamwaya, M. E.; Papavinasam, E.; Teoh, S. G.; Rajaram, R. K. Z. Kristallogr. 1984, 169, 51.

(34) Démaret, A.; Abraham, F. Acta Crystallogr., Sect. C: Cryst. Struct. Commun. 1987, 43, 1519.

(35) Nomiya, K.; Takahashi, S.; Noguchi, R.; Nemoto, S.; Takayama, T.; Oda, M. Inorg. Chem. 2000, 39, 3301.

(36) Nomiya, K.; Yokoyama, H. J. Chem. Soc., Dalton Trans. 2002 2483.

(37) Wang, L.; Pei, Y. Acta Crystallogr., Sect. E: Struct. Rep. Online 2006, 62, m1487.

(38) Kasuga, N. C.; Sato, M.; Amano, A.; Hara, A.; Tsuruta, S.; Sugie, A.; Nomiya, K. Inorg. Chim. Acta 2008, 361, 1267.

(39) Kasuga, N. C.; Takagi, Y.; Tsuruta, S.-i.; Kuwana, W.; Yoshikawa, R.; Nomiya, K. Inorg. Chim. Acta 2011, 368, 44.

(40) Kasuga, N. C.; Yoshikawa, R.; Sakai, Y.; Nomiya, K. Inorg. Chem. 2012, 51, 1640 .

(41) Mirolo, L.; Schmidt, T.; Eckhardt, S.; Meuwly, M.; Fromm, K. M. Chem.-Eur. J. 2012, 18, in press, DOI:10.1002/chem.201201844.

(1) Fromm, K. M. Nat. Chem. 2011, 3, 178.
(2) Huh, A. J.; Kwon, Y. J. J. Controlled Release 2011, 156, 128. 
(42) Orpen, A. G.; Brammer, L.; Allen, F. H.; Kennard, O.; Watson, D. G.; Taylor, R. J. Chem. Soc., Dalton Trans. 1989, 12, S1-S83.

(43) Jansen, M. Angew. Chem., Int. Ed. Engl. 1987, 26, 1098.

(44) Pyykkö, P. Chem. Rev. 1997, 97, 597.

(45) Bondi, A. J. Phys. Chem. 1964, 68, 441.

(46) Holleman, A. F.; Wiberg, E.; Wiberg, N. Lehrbuch der Anorganischen Chemie; de Gruyter: Berlin, 2007.

(47) Shoeib, T.; Sharp, B. L. Inorg. Chim. Acta 2009, 362, 1925.

(48) Ahmad, S.; Hanif, M.; Monim-ul-Mehboob, M.; Isab, A.; Ahmad, S. Synth. React. Inorg., Met.-Org., Nano-Met. Chem. 2009, 39, 45 .

(49) Mantion, A.; Taubert, A. In Encyclopedia of Supramolecular Chemistry: Metal-Peptide Frameworks; Atwood, J., Steed, J., Wallace, K., Eds.; Taylor \& Francis: New York, 2012.

(50) Li, H.; Siu, K. W. M.; Guevremont, R.; Le Blanc, J. C. Y. J. Am. Soc. Mass Spectrom 1997, 8, 781.

(51) Chu, I. K.; Guo, X.; Lau, T.-C.; Siu, K. W. M. Anal. Chem. 1999, $71,2364$.

(52) Chu, I. K.; Shoeib, T.; Guo, X.; Rodriquez, C. F.; Hopkinson, A. C.; Siu, K. W. M.; Lau, T.-C. J. Am. Soc. Mass Spectrom. 2001, 12, 163.

(53) Chu, I. K.; Cox, D. M.; Guo, X.; Kireeva, I.; Lau, T.-C.; McDermott, J. C.; Siu, K. W. M. Anal. Chem. 2002, 74, 2072.

(54) Jayasekharan, T.; Sahoo, N. K. Rapid Commun. Mass Spectrom. 2010, 24, 3562.

(55) Nan, J.; Yan, X.-P. Chem.-Eur. J. 2010, 16, 423.

(56) McCully, K. S. Nat. Med. 1996, 2, 386.

(57) Focks, J. J.; Clappers, N.; Brouwer, M. A.; Cramer, E. G.; van Dijk, E. G.; van Oijen, M. G.; Jansen, J. B.; Peters, W. H. M.; Verheugt, F. W. J. Am. Coll. Cardiol. 2010, 55, A175-E1643.

(58) Shen, J.-S.; Li, D.-H.; Zhang, M.-B.; Zhou, J.; Zhang, H.; Jiang, Y.-B. Langmuir 2011, 27, 481.

(59) Feng, D.-Q.; Liu, G.; Zheng, W.; Liu, J.; Chen, T.; Li, D. Chem. Commun. 2011, 47, 8557.

(60) Gorsuch, J. W.; Klaine, S. J. Environ. Toxicol. Chem. 1998, 17, 537.

(61) Mejáre, M.; Bülow, L. Trends Biotechnol. 2001, 19, 67.

(62) Scheuhammer, A. M.; Cherian, M. G. Toxicol. Appl. Pharmacol. 1986, 82, 417.

(63) Margoshes, M.; Vallee, B. L. J. Am. Chem. Soc. 1957, 79, 4813.

(64) Klaassen, C. D. Metallothionein IV; Birkhäuser: Basel, 1999.

(65) Kaegi, J. H. R.; Kojima, Y. Exp. Suppl. 1987, 52, 25.

(66) Blindauer, C. A.; Leszczyszyn, O. I. Nat. Prod. Rep. 2010, 27, 720 .

(67) Riedel, E. Anorganische Chemie; de Gruyter: Berlin, 1999.

(68) Peterson, C. W.; Narula, S. S.; Armitage, I. M. FEBS Lett. 1996, 379,85 .

(69) The number indicates the position of the amino acid, the primary sequence, and was neither subscripted nor superscripted to avoid confusion with chemical formula or references, respectively.

(70) González-Bellavista, A.; Atrian, S.; Muñoz, M.; Capdevila, M.; Fàbregas, E. Talanta 2009, 77, 1528.

(71) Krizkova, S.; Huska, D.; Beklova, M.; Hubalek, J.; Adam, V.; Trnkova, L.; Kizek, R. Environ. Toxicol. Chem. 2010, 29, 492.

(72) Kobylinska, D. K.; Bruzzoniti, M. C.; Sarzanini, C.; Franko, M. J. Phys.: Conf. Ser. 2010, 214, 12119.

(73) Trnkova, L.; Krizkova, S.; Adam, V.; Hubalek, J.; Kizek, R. Biosens. Bioelectron. 2011, 26, 2201.

(74) Vasak, M. Methods Enzymol. 1991, 205, 452.

(75) Robinson, N. J.; Tommey, A. M.; Kuske, C.; Jackson, P. J. Biochem. J. 1993, 295, 1.

(76) Zenk, M. H. Gene 1996, 179, 21.

(77) Mikelova, R.; Baloun, J.; Petrlova, J.; Adam, V.; Havel, L.; Petrek, J.; Horna, A.; Kizek, R. Bioelectrochemistry 2007, 70, 508.

(78) Ortiz, D. F.; Ruscitti, T.; McCue, K. F.; Ow, D. W. J. Biol. Chem. 1995, 270, 4721.

(79) Barbucci, R.; Odriozola, I.; Ormategui, N.; Loinaz, I.; Pomposo, J. A.; Grande, H. J. Macromol. Symp. 2008, 266, 96.

(80) Casuso, P.; Carrasco, P.; Loinaz, I.; Cabanero, G.; Grande, H. J.; Odriozola, I. Soft Matter 2011, 7, 3627.
(81) Casuso, P.; Carrasco, P.; Loinaz, I.; Grande, H. J.; Odriozola, I. Org. Biomol. Chem. 2010, 8, 5455.

(82) Shen, J.-S.; Li, D.-H.; Cai, Q.-G.; Jiang, Y.-B. J. Mater. Chem. 2009, 19, 6219.

(83) Li, D.-H.; Shen, J.-S.; Chen, N.; Ruan, Y.-B.; Jiang, Y.-B. Chem. Commun. 2011, 47, 5900.

(84) Liu, Y.; Ma, W.; Liu, W.; Li, C.; Liu, Y.; Jiang, X.; Tang, Z. J. Mater. Chem. 2011, 21, 19214.

(85) Slocik, J. M.; Wright, D. W. Biomacromolecules 2003, 4, 1135.

(86) Moshe, A.; Markovich, G. Chem. Mater. 2011, 23, 1239.

(87) Wu, Q.; Cao, H.; Luan, Q.; Zhang, J.; Wang, Z.; Warner, J. H.; Watt, A. Inorg. Chem. 2008, 47, 5882.

(88) Kumar, A. S.; Abyaneh, M. K.; Gosavi, S. W.; Kulkarni, S. K.; Pasricha, R.; Ahmad, A.; Khan, M. I. Biotechnol. Lett. 2007, 29, 439.

(89) Santillan, M. J.; Quaranta, N. E.; Boccaccini, A. R. Surf. Coat. Technol. 2010, 205, 2562.

(90) Krumov, N.; Perner-Nochta, I.; Oder, S.; Gotcheva, V.; Angelov, A.; Posten, C. Chem. Eng. Technol. 2009, 32, 1026.

(91) Dickerson, M. B.; Sandhage, K. H.; Naik, R. R. Chem. Rev. 2008, 108,4935

(92) Chen, C.-L.; Rosi, N. L. Angew. Chem., Int. Ed. 2010, 49, 1924.

(93) Davis, M. E. Science 2004, 305, 480.

(94) Klaus, T.; Joerger, R.; Olsson, E.; Granquist, C.-G. Proc. Natl. Acad. Sci. U.S.A. 1999, 96, 13611.

(95) Moulton, M. C.; Braydich-Stolle, L. K.; Nadagouda, M. N.; Kunzelman, S.; Hussain, S. M.; Varma, R. S. Nanoscale 2010, 2, 763.

(96) Nadagouda, M. N.; Varma, R. S. Green Chem. 2008, 10, 859.

(97) Smith, G. P. Science 1985, 228, 1315.

(98) Seker, U. O. S.; Demir, H. V. Molecules 2011, 16, 1426.

(99) Rodi, D. J.; Makowski, L. Curr. Opin. Biotechnol. 1999, 10, 87.

(100) Naik, R. R.; Jones, S. E.; Murray, C. J.; McAuliffe, J. C.; Vaia, R. A.; Stone, M. O. Adv. Funct. Mater. 2004, 14, 25.

(101) Naik, R. R.; Stringer, S. J.; Agarwal, G.; Jones, S. E.; Stone, M. O. Nat. Mater. 2002, 1, 169.

(102) Bassindale, A. R.; Codina-Barrios, A.; Frascione, N.; Taylor, P. G. Chem. Commun. 2007, 28, 2956.

(103) Lee, E.; Kim, D.-H.; Woo, Y.; Hur, H.-G.; Lim, Y. Biochem. Biophys. Res. Commun. 2008, 376, 595.

(104) Sengupta, A.; Thai, C. K.; Sastry, M. S. R.; Matthaei, J. F.; Schwartz, D. T.; Davis, E. J.; Baneyx, F. Langmuir 2008, 24, 2000.

(105) Ostrov, N.; Gazit, E. Angew. Chem., Int. Ed. 2010, 49, 3018.

(106) Sano, K.-I.; Sasaki, H.; Shiba, K. Langmuir 2005, 21, 3090.

(107) Sedlak, H. R.; Hnilova, M.; Grosh, C.; Fong, H.; Baneyx, F.; Schwartz, D.; Sarikaya, M.; Tamerler, C.; Traxler, B. Appl. Environ. Microbiol. 2012, 78, 2289.

(108) Xie, J.; Lee, J. Y.; Wang, D. I. C.; Ting, Y. P. ACS Nano 2007, $1,429$.

(109) Selvakannan, P. R.; Swami, A.; Srisathiyanarayanan, D.; Shirude, P. S.; Pasricha, R.; Mandale, A. B.; Sastry, M. Langmuir 2004, 20, 7825.

(110) Dong, Q.; Su, H.; Cao, W.; Han, J.; Di, Z.; Guo, Q. Mater. Chem. Phys. 2008, 110, 160.

(111) Stubbe, J.; Nocera, D. G.; Yee, C. S.; Chang, M. C. Y. Chem. Rev. 2003, 103, 2167.

(112) Gao, J.; Müller, P.; Wang, M.; Eckhardt, S.; Lauz, M.; Fromm, K. M.; Giese, B. Angew. Chem., Int. Ed. 2011, 50, 1926.

(113) Thompson, D. C.; Perera, K.; London, R. Chem. Res. Toxicol. $1995,8,55$.

(114) Ascik, K. Przegl. Papier. 1973, 29, 239.

(115) Si, S.; Bhattacharjee, R. R.; Banerjee, A.; Mandal, T. K. Chem.Eur. J. 2006, 12, 1256.

(116) Malencik, D. A.; Anderson, S. R. Amino Acids 2003, 25, 233.

(117) Sjödin, M.; Styring, S.; Åkermark, B.; Sun, L.; Hammarström, L. J. Am. Chem. Soc. 2000, 122, 3932.

(118) Belser, K.; Vig Slenters, T.; Pfumbidzai, C.; Upert, G.; Mirolo, L.; Fromm, K. M.; Wennemers, H. Angew. Chem., Int. Ed. 2009, 48, 3661.

(119) Henglein, A. J. Phys. Chem. 1993, 97, 5457. 
(120) Fischer, V.; Landfester, K.; Muñoz-Espí, R. Cryst. Growth Des. 2011, 11, 1880 .

(121) Nam, K. T.; Lee, Y. J.; Krauland, E. M.; Kottmann, S. T.; Belcher, A. M. ACS Nano 2008, 2, 1480.

(122) Roy, S.; Banerjee, A. Soft Matter 2011, 7, 5300.

(123) Adhikari, B.; Banerjee, A. Chem.-Eur. J. 2010, 16, 13698.

(124) Carter, C. J.; Ackerson, C. J.; Feldheim, D. L. ACS Nano 2010, 4, 3883.

(125) Lee, Y. J.; Lee, Y.; Oh, D.; Chen, T.; Ceder, G.; Belcher, A. M. Nano Lett. 2010, 10, 2433.

(126) Giese, B.; Eckhardt, S.; Lauz, M. In Encyclopedia of Radicals in Chemistry, Biology and Materials; Chatgilialoglu, C., Studer, A., Eds.; John Wiley \& Sons: Chichester, West Sussex, Hoboken, NJ, 2012.

(127) Si, S.; Mandal, T. K. Chem.-Eur. J. 2007, 13, 3160.

(128) Selvakannan, P. R.; Mandal, S.; Phadtare, S.; Gole, A.; Pasricha, R.; Adyanthaya, S. D.; Sastry, M. J. Colloid Interface Sci. 2004, 269, 97.

(129) Dickerson, M. B.; Naik, R. R.; Stone, M. O.; Cai, Y.; Sandhage,

K. H. Chem. Commun. 2004, 15, 1776.

(130) Kulesza, A.; Mitrić, R.; Bonačić-Koutecký, V.; Bellina, B.; Compagnon, I.; Broyer, M.; Antoine, R.; Dugourd, P. Angew. Chem., Int. Ed. 2011, 50, 878.

(131) Khan, Z.; Talib, A. Colloids Surf., B 2010, 76, 164.

(132) Mantion, A.; Guex, A. G.; Foelske, A.; Mirolo, L.; Fromm, K. M.; Painsi, M.; Taubert, A. Soft Matter 2008, 4, 606.

(133) Li, S.; Shen, Y.; Xie, A.; Yu, X.; Qiu, L.; Zhang, L.; Zhang, Q. Green Chem. 2007, 9, 852.

(134) van den Berg, A. J. J.; Horsten, S. F. A. J.; Kettenes-van den Bosch, J. J.; Kroes, B. H.; Beukelman, C. J.; Leeflang, B. R.; Labadie, R. P. FEBS Lett. 1995, 358, 215.

(135) Auvin, C.; Baraguey, C.; Blond, A.; Lezenven, F.; Pousset, J.-L.; Bodo, B. Tetrahedron Lett. 1997, 38, 2845.

(136) Bar, H.; Bhui, D. K.; Sahoo, G. P.; Sarkar, P.; De, S. P.; Misra, A. Colloids Surf., A 2009, 339, 134.

(137) Bar, H.; Bhui, D. K.; Sahoo, G. P.; Sarkar, P.; Pyne, S.; Misra, A. Colloids Surf., A 2009, 348, 212.

(138) Sanghi, R.; Verma, P. Bioresour. Technol. 2009, 100, 501.

(139) Eby, D. M.; Schaeublin, N. M.; Farrington, K. E.; Hussain, S. M.; Johnson, G. R. ACS Nano 2009, 3, 984.

(140) Eby, D. M.; Luckarift, H. R.; Johnson, G. R. ACS Appl. Mater. Interfaces 2009, 1, 1553.

(141) Durán, N.; Marcato, P. D.; Durán, M.; Yadav, A.; Gade, A.; Rai, M. Appl. Microbiol. Biotechnol. 2011, 90, 1609.

(142) Gazit, E. Chem. Soc. Rev. 2007, 36, 1263.

(143) Díez, I.; Hahn, H.; Ikkala, O.; Börner, H. G.; Ras, R. H. A. Soft Matter 2010, 6, 3160.

(144) Reches, M.; Gazit, E. Science 2003, 300, 625.

(145) Carny, O.; Shalev, D. E.; Gazit, E. Nano Lett. 2006, 6, 1594.

(146) Bale, S. S.; Asuri, P.; Karajanagi, S. S.; Dordick, J. S.; Kane, R. S. Adv. Mater. 2007, 19, 3167.

(147) Brust, M.; Walker, M.; Bethell, D.; Schiffrin, D. J.; Whyman, R. J. Chem. Soc., Chem. Commun. 1994, 7, 801.

(148) Graf, P.; Mantion, A.; Foelske, A.; Shkilnyy, A.; Mašić, A.; Thünemann, A. F.; Taubert, A. Chem.-Eur. J. 2009, 15, 5831.

(149) Graf, P.; Mantion, A.; Haase, A.; Thünemann, A. F.; Mašić, A.; Meier, W.; Luch, A.; Taubert, A. ACS Nano 2011, 5, 820.

(150) Mantion, A.; Graf, P.; Florea, I.; Haase, A.; Thünemann, A. F.; Mašić, A.; Ersen, O.; Rabu, P.; Meier, W.; Luch, A.; Taubert, A. Nanoscale 2011, 3, 5168.

(151) Upert, G.; Bouillère, F.; Wennemers, H. Angew. Chem., Int. Ed. 2012, 51, 4231 .

(152) Morones, J. R.; Elechiguerra, J. L.; Camacho, A.; Holt, K.; Kouri, J. B.; Ramírez, J. T.; Yacaman, M. J. Nanotechnology 2005, 16, 2346.

(153) Zhao, G.; Stevens, S. E. BioMetals 1998, 11, 27.

(154) Slawson, R. M.; van Dyke, M. I.; Lee, H.; Trevors, J. T. Plasmid 1992, 27, 72.

(155) Lok, C.-N.; Ho, C.-M.; Chen, R.; He, Q.-Y.; Yu, W.-Y.; Sun, H.; Tam, P. K.-H.; Chiu, J.-F.; Che, C.-M. J. Biol. Inorg. Chem. 2007, $12,527$.
(156) Kannan, N.; Subbalaxmi, S. Rev. Adv. Mater. Sci. 2011, 27, 99. (157) Baker, C.; Pradhan, A.; Pakstis, L.; Pochan, D. J.; Shah, S. I. J. Nanosci. Nanotechnol. 2005, 5, 244.

(158) Panáček, A.; Kvítek, L.; Prucek, R.; Kolář, M.; Večeřová, R.; Pizúrová, N.; Sharma, V. K.; Navěčná, T.; Zbořil, R. J. Phys. Chem. 2006, 110, 16248.

(159) Thakkar, K. N.; Mhatre, S. S.; Parikh, R. Y. Nanomedicine 2010, $6,257$.

(160) Samberg, M. E.; Orndorff, P. E.; Monteiro-Riviere, N. A. Nanotoxicology 2011, 5, 244.

(161) Lok, C.-N.; Ho, C.-M.; Chen, R.; He, Q.-Y.; Yu, W.-Y.; Sun, H.; Tam, P. K.-H.; Chiu, J.-F.; Che, C.-M. J. Proteome Res. 2006, 5, 916.

(162) Li, W.-R.; Xie, X.-B.; Shi, Q.-S.; Duan, S.-S.; Ouyang, Y.-S.; Chen, Y.-B. BioMetals 2011, 24, 135.

(163) Sadhasivam, S.; Shanmugam, P.; Yun, K. Colloids Surf., B 2010, 81,358 .

(164) Kathiresan, K.; Alikunhi, N. M.; Pathmanaban, S.; Nabikhan, A.; Kandasamy, S. Can. J. Microbiol. 2010, 56, 1050.

(165) Li, W.-R.; Xie, X.-B.; Shi, Q.-S.; Zeng, H.-Y.; Ouyang, Y.-S.; Chen, Y.-B. Appl. Microbiol. Biotechnol. 2010, 85, 1115.

(166) Kim, J. S.; Kuk, E.; Yu, K. N.; Kim, J.-H.; Park, S. J.; Lee, H. J.; Kim, S. H.; Park, Y. K.; Park, Y. H.; Hwang, C.-Y.; Kim, Y.-K.; Lee, Y.S.; Jeong, D. H.; Cho, M.-H. Nanomedicine 2007, 3, 95.

(167) Elechiguerra, J. L.; Burt, J. L.; Morones, J. R.; CamachoBragado, A.; Gao, X.; Lara, H. H.; Yacaman, M. J. J. Nanobiotechnol. 2005, 3, 6.

(168) Sun, R. W.-Y.; Chen, R.; Chung, N. P.-Y.; Ho, C.-M.; Lin, C.-L. S.; Che, C.-M. Chem. Commun. 2005, 40, 5059.

(169) Lara, H. H.; Ayala-Nuñez, N. V.; Ixtepan-Turrent, L.; Rodriguez-Padilla, C. J. Nanobiotechnol. 2010, 8, 1.

(170) Lu, L.; Sun, R. W.-Y.; Chen, R.; Hui, C.-K.; Ho, C.-M.; Luk, J. M.; Lau, G. K. K.; Che, C.-M. Antiviral Ther. 2008, 13, 253.

(171) Baram-Pinto, D.; Shukla, S.; Perkas, N.; Gedanken, A.; Sarid, R. Bioconjugate Chem. 2009, 20, 1497.

(172) Rogers, J. V.; Parkinson, C. V.; Choi, Y. W.; Speshock, J. L.; Hussain, S. M. Nanoscale Res. Lett. 2008, 3, 129.

(173) Sun, L.; Singh, A. K.; Vig, K.; Pillai, S. R.; Singh, S. R. J. Biomed. Nanotechnol. 2008, 4, 149.

(174) Hwang, E. T.; Lee, J. H.; Chae, Y. J.; Kim, Y. S.; Kim, B. C.; Sang, B.-I.; Gu, M. B. Small 2008, 4, 746.

(175) Feng, Q. L.; Wu, J.; Chen, G. Q.; Cui, G. Q.; Kim, T. N.; Kim, J. O. J. Biomed. Mater. Res. 2000, 52, 662.

(176) Marambio-Jones, C.; Hoek, E. M. V. J. Nanopart. Res. 2010, 12, 1531.

(177) Levinson, W. Review of Medical Microbiology and Immunology; McGraw-Hill Professional: New York, 2006.

(178) Jung, W. K.; Koo, H. C.; Kim, K. W.; Shin, S.; Kim, S. H.; Park, Y. H. Appl. Environ. Microbiol. 2008, 74, 2171.

(179) Choi, O.; Deng, K. K.; Kim, N.-J.; Ross, L.; Surampalli, R. Y.; Hu, Z. Water Res. 2008, 42, 3066.

(180) Pal, S.; Tak, Y. K.; Song, J. M. Appl. Environ. Microbiol. 2007, 73, 1712 .

(181) El Badawy, A. M.; Silva, R. G.; Morris, B.; Scheckel, K. G.; Suidan, M. T.; Tolaymat, T. M. Environ. Sci. Technol. 2011, 45, 283.

(182) Sui, Z.; Chen, X.; Wang, L.; Zhuang, W.; Chai, Y.; Yang, C. Phys. E (Amsterdam, Neth.) 2006, 33, 308.

(183) Fayaz, A. M.; Balaji, K.; Girilal, M.; Yadav, R.; Kalaichelvan, P. T.; Venketesan, R. Nanomedicine 2010, 6, 103.

(184) van der Wal, A.; Norde, W.; Zehnder, A. J.; Lyklema, J. Colloids Surf., B 1997, 9, 81 .

(185) Mirzajani, F.; Ghassempour, A.; Aliahmadi, A.; Esmaeili, M. A. Res. Microbiol. 2011, 162, 542.

(186) Sintubin, L.; de Gusseme, B.; van der Meeren, P.; Pycke, B. F. G.; Verstraete, W.; Boon, N. Appl. Microbiol. Biotechnol. 2011, 91, 153. (187) Neal, A. L. Ecotoxicology 2008, 17, 362.

(188) Quadros, M.; Marr, L. C. J. Air Waste Manage. Assoc. 2010, 60, 770 . 
(189) Jaiswal, S.; Duffy, B.; Jaiswal, A. K.; Stobie, N.; McHale, P. Int. J. Antimicrob. Agents 2010, 36, 280.

(190) Liu, J.; Hurt, R. H. Environ. Sci. Technol. 2010, 44, 2169.

(191) Xiu, Z.-M.; Ma, J.; Alvarez, P. J. J. Environ. Sci. Technol. 2011, 45, 9003.

(192) AshaRani, P. V.; Low Kah Mun, G.; Hande, M. P.; Valiyaveettil, S. ACS Nano 2009, 3, 279.

(193) Liu, J.; Sonshine, D. A.; Shervani, S.; Hurt, R. H. ACS Nano 2010, 4, 6903.

(194) http://www.saltlakemetals.com/SolubilityProducts.htm.

(195) Kaegi, R.; Voegelin, A.; Sinnet, B.; Zuleeg, S.; Hagendorfer, H.; Burkhardt, M.; Siegrist, H. Environ. Sci. Technol. 2011, 45, 3902.

(196) Ratte, H. T. Environ. Toxicol. Chem. 1999, 18, 89.

(197) Wang, J.; Huang, C.; Pirestani, D. Water Res. 2003, 37, 4444.

(198) Choi, O.; Clevenger, T. E.; Deng, B.; Surampalli, R. Y.; Ross, J.

L.; Hu, Z. Water Res. 2009, 43, 1879.

(199) Holt, K. B.; Bard, A. J. Biochemistry 2005, 44, 13214.

(200) Arakawa, H.; Neault, J. F.; Tajmir-Riahi, H. A. Biophys. J. 2001, 81,1580 .

(201) Johannsen, S.; Megger, N.; Böhme, D.; SigelRoland, K. O.;

Müller, J. Nat. Chem. 2010, 2, 229.

(202) Binder, B. M. Plant Sci. 2008, 175, 8.

(203) Chen, X.; Schluesener, H. J. Toxicol. Lett. 2008, 176, 1.

(204) Liau, S. Y.; Read, D. C.; Pugh, W. J.; Furr, J. R.; Russell, A. D. Lett. Appl. Microbiol. 1997, 25, 279.

(205) Russel, A. D.; Path, F. R. C.; Pharm, F. R.; Hugo, W. B. Prog. Med. Chem. 1994, 31, 351.

(206) Xu, F. F.; Imlay, J. A. Appl. Environ. Microbiol. 2012, 78, 3614.

(207) Gordon, O.; Vig Slenters, T.; Brunetto, P. S.; Villaruz, A. E.;

Sturdevant, D. E.; Otto, M.; Landmann, R.; Fromm, K. M. Antimicrob. Agents Chemother. 2010, 54, 4208.

(208) Kim, J. Y.; Lee, C.; Cho, M.; Yoon, J. Water Res. 2008, 42, 356.

(209) Bragg, P. D.; Rainnie, D. J. Can. J. Microbiol. 1974, 20, 883.

(210) Dibrov, P.; Dzioba, J.; Gosink, K. K.; Häse, C. C. Antimicrob. Agents Chemother. 2002, 46, 2668.

(211) Park, H.-J.; Kim, J. Y.; Kim, J.; Lee, J.-H.; Hahn, J.-S.; Gu, M. B.; Yoon, J. Water Res. 2009, 43, 1027.

(212) Cabiscol, E.; Tamarit, J.; Ros, J. Int. Microbiol. 2000, 3 , 3.

(213) Choi, O.; Hu, Z. Environ. Sci. Technol. 2008, 42, 4583.

(214) Inoue, Y.; Hoshino, M.; Takahashi, H.; Noguchi, T.; Murata, T.; Kanzaki, Y.; Hamashima, H.; Sasatsu, M. J. Inorg. Biochem. 2002, 92, 37.

(215) Wang, H.; Law, N.; Pearson, G.; van Dongen, B. E.; Jarvis, R. M.; Goodacre, R.; Lloyd, J. R. J. Bacteriol. 2010, 192, 1143.

(216) Parikh, R. Y.; Singh, S.; Prasad, B. L. V.; Patole, M. S.; Sastry, M.; Shouche, Y. S. ChemBioChem 2008, 9, 1415.

(217) Gupta, A.; Matsui, K.; Lo, J.-F.; Silver, S. Nat. Med. 1999, 5, 183.

(218) Klaus-Joerger, T.; Joerger, R.; Olsson, E.; Granqvist, C.-G. Trends Biotechnol. 2001, 19, 15.

(219) Percival, S.; Bowler, P.; Russell, D. J. Hosp. Infect. 2005, 60, 1.

(220) Loh, J. V.; Percival, S. L.; Woods, E. J.; Williams, N. J.; Cochrane, C. A. Int. Wound J. 2009, 6, 32.

(221) Chopra, I. J. Antimicrob. Chemother. 2007, 59, 587.

(222) Cason, J. S.; Jackson, D. M.; Lowbury, E. J.; Ricketts, C. R. Br. Med. J. 1966, 2, 1288.

(223) McHugh, G. L.; Moellering, R. C.; Hopkins, C. C.; Swartz, M. N. Lancet 1975, 1, 235 .

(224) Hendry, A. T.; Stewart, I. O. Can. J. Microbiol. 1979, 25, 915. (225) Annear, D. I.; Mee, B. J.; Bailey, M. J. Clin. Pathol. 1976, 29, 441.

(226) Bridges, K.; Kidson, A.; Lowbury, E. J.; Wilkins, M. D. Br. Med. J. 1979, 1, 446.

(227) Coward, J. E.; Carr, H. S.; Rosenkranz, H. S. Antimicrob. Agents Chemother. 1973, 3, 621.

(228) Rosenkranz, H. S.; Coward, J. E.; Wlodkowski, T. J.; Carr, H. S. Antimicrob. Agents Chemother. 1974, 5, 199.

(229) Silver, S. J. Ind. Microbiol. Biotechnol. 1998, 20, 1.
(230) Silver, S.; Le Phung, T. J. Ind. Microbiol. Biotechnol. 2005, 32, 587.

(231) Trevors, J. T.; Starodub, M. E. Curr. Microbiol. 1990, 21, 103.

(232) Deshpande, L. M.; Chopade, B. A. BioMetals 1994, 7, 49.

(233) Franke, S.; Grass, G.; Nies, D. H. Microbiology 2001, 147, 965.

(234) Silvestry-Rodriguez, N.; Sicairos-Ruelas, E. E.; Gerba, C. P.; Bright, K. R. Rev. Environ. Contam. Toxicol. 2007, 191, 23.

(235) Foldbjerg, R.; Olesen, P.; Hougaard, M.; Dang, D. A.; Hoffmann, H. J.; Autrup, H. Toxicol. Lett. 2009, 190, 156.

(236) Haefeli, C.; Franklin, F.; Hardy, K. J. Bacteriol. 1984, 158, 389.

(237) Slawson, R. M.; Trevors, J. T.; Lee, H. Arch. Microbiol. 1992, $158,398$.

(238) Silver, S.; Gupta, A.; Matsui, K.; Lo, J.-F. Met.-Based Drugs 1999, 6, 4.

(239) Nies, D. H. FEMS Microbiol. Rev. 2003, 27, 313.

(240) Parikh, R. Y.; Ramanathan, R.; Coloe, P. J.; Bhargava, S. K.; Patole, M. S.; Shouche, Y. S.; Bansal, V. PLoS One 2011, 6, 1.

(241) Lok, C.-N.; Ho, C.-M.; Chen, R.; Tam, P. K.-H.; Chiu, J.-F.; Che, C.-M. J. Proteome Res. 2008, 7, 2351.

(242) Su, C.-C.; Long, F.; Yu, E. W. Protein Sci. 2011, 20, 6.

(243) Loftin, I. R.; Franke, S.; Blackburn, N. J.; McEvoy, M. M. Protein Sci. 2007, 16, 2287.

(244) Loftin, I. R.; Franke, S.; Roberts, S. A.; Weichsel, A.; Héroux, A.; Montfort, W. R.; Rensing, C.; McEvoy, M. M. Biochemistry 2005, 44, 10533.

(245) Kittleson, J. T.; Loftin, I. R.; Hausrath, A. C.; Engelhardt, K. P.; Rensing, C.; McEvoy, M. M. Biochemistry 2006, 45, 11096.

(246) Kim, E.-H.; Rensinga, C.; McEvoy, M. M. Nat. Prod. Rep. 2010, 27, 711.

(247) http://www.uniprot.org/uniprot/P77214.

(248) http://www.uniprot.org/uniprot/F5RX87.

(249) Ramanathan, R.; O’Mullane, A. P.; Parikh, R. Y.; Smooker, P. M.; Bhargava, S. K.; Bansal, V. Langmuir 2011, 27, 714.

(250) Sharma, V.; Yngard, R.; Lin, Y. Adv. Colloid Interface Sci. 2009, $145,83$.

(251) Juibari, M. M.; Abbasalizadeh, S.; Jouzani, G. S.; Noruzi, M. Mater. Lett. 2011, 65, 1014.

(252) Gurunathana, S.; Kalishwaralala, K.; Vaidyanathana, R.; Deepak, V.; Pandiana, S. R. K.; Muniyandia, J.; Hariharana, N.; Eomb, S. H. Colloids Surf., B 2009, 74, 328.

(253) Sintubin, L.; Windt, W. de; Dick, J.; Mast, J.; van der Ha, D.; Verstraete, W.; Boon, N. Appl. Microbiol. Biotechnol. 2009, 84, 741.

(254) Nair, B.; Pradeep, T. Cryst. Growth Des. 2002, 2, 293.

(255) Kalimuthu, K.; Babu, R. S.; Venkataraman, D.; Bilal, M.; Gurunathan, S. Colloids Surf., B 2008, 65, 150.

(256) Vaidyanathan, R.; Gopalram, S.; Kalishwaralal, K.; Deepak, V.; Pandian, S. R. K.; Gurunathan, S. Colloids Surf., B 2010, 75, 335.

(257) Shahverdi, A. R.; Minaeian, S.; Shahverdi, H. R.; Jamalifar, H.; Nohi, A.-A. Process Biochem. 2007, 42, 919.

(258) Law, N.; Ansari, S.; Livens, F. R.; Renshaw, J. C.; Lloyd, J. R. Appl. Environ. Microbiol. 2008, 74, 7090.

(259) Veazey, J.; Reguera, G.; Tessmer, S. Phys. Rev. E 2011, 84, 60901.

(260) Feliciano, G. T.; da Silva, A. J. R.; Reguera, G.; Artacho, E. J. Phys. Chem. A 2012, 116, 8023.

(261) Bhainsa, K. C.; D’Souza, S. Colloids Surf., B 2006, 47, 160.

(262) Navazi, Z. R.; Pazouki, M.; Halek, F. S. Iran. J. Biotechnol. 2010, $8,56$.

(263) Balaji, D.; Basavaraja, S.; Deshpande, R.; Bedre Mahesh, D.; Prabhakar, B.; Venkataraman, A. Colloids Surf., B 2009, 68, 88.

(264) Durán, N.; Marcato, P. D.; Alves, O. L.; Souza, G. I. H. de; Esposito, E. J. Nanobiotechnol. 2005, 3, 8.

(265) Kowshik, M.; Ashtaputre, S.; Kharrazi, S.; Vogel, W.; Urban, J.; Kulkarni, S. K.; Paknikar, K. M. Nanotechnology 2003, 14, 95.

(266) Daizy, P. Spectrochim. Acta, Part A 2009, 73, 374.

(267) Kathiresan, K.; Manivannan, S.; Nabeel, M.; Dhivya, B. Colloids Surf., B 2009, 71, 133.

(268) Shaligram, N. S.; Bule, M.; Bhambure, R.; Singhal, R. S.; Singh, S. K.; Szakacs, G.; Pandey, A. Process Biochem. 2009, 44, 939. 
(269) Vigneshwaran, N.; Kathe, A. A.; Varadarajan, P.; Nachane, R. P.; Balasubramanya, R. Colloids Surf., B 2006, 53, 55.

(270) Chen, J.; Lin, Z.; Ma, X. Lett. Appl. Microbiol. 2003, 37, 105.

(271) Thirumurugan, G.; Shaheedha, S.; Dhanaraju, M. Int. J. ChemTech Res. 2009, 1, 714.

(272) Ganesh Babu, M.; Gunasekaran, P. Colloids Surf., B 2009, 74, 191.

(273) Kalishwaralal, K.; Deepak, V.; Ramkumarpandian, S.; Nellaiah, H.; Sangiliyandi, G. Mater. Lett. 2008, 62, 4411.

(274) Pugazhenthiran, N.; Anandan, S.; Kathiravan, G.; Prakash, N. K. U.; Crawford, S.; Ashokkumar, M. J. Nanopart. Res. 2009, 11, 1811.

(275) Kalishwaralal, K.; Deepak, V.; Pandian, S. R. K.; Kottaisamy, M.; Kanth, S. B.; Kartikeyan, B.; Gurunathan, S. Colloids Surf., B 2010, 77, 257.

(276) Zhang, J.; Xu, S.; Kumacheva, E. Adv. Mater. 2005, 17, 2336.

(277) Zhang, H.; Li, Q.; Wang, H.; Sun, D.; Lu, Y.; He, N. Appl. Biochem. Biotechnol. 2007, 143, 54.

(278) Fu, M.; Li, Q.; Sun, D.; Yinghua, L.; Ning, H.; Xu, D.; Huixuan, W.; Huang, J. Chin. J. Chem. Eng. 2006, 14, 114.

(279) Joerger, R.; Klaus, T.; Granqvist, C.-G. Adv. Mater. 2000, 12, 407.

(280) Lengke, M. F.; Fleet, M. E.; Southam, G. Langmuir 2007, 23, 2694.

(281) Gardea-Torresdey, J. L.; Gomez, E.; Peralta-Videa, J. R.; Parsons, J. G.; Troiani, H.; Jose-Yacaman, M. Langmuir 2003, 19, 1357.

(282) Tripathy, A.; Raichur, A. M.; Chandrasekaran, N.; Prathna, T. C.; Mukherjee, A. J. Nanopart. Res. 2010, 12, 237.

(283) Huang, J.; Li, Q.; Sun, D.; Lu, Y.; Su, Y.; Yang, X.; Wang, H.; Wang, Y.; Shao, W.; He, N.; Hong, J.; Chen, C. Nanotechnology 2007, $18,1$.

(284) Lin, Z.; Zhou, C.; Wu, J.; Zhou, J.; Wang, L. Spectrochim. Acta, Part A 2005, 61, 1195.

(285) Zhang, H.; Li, Q.; Lu, Y.; Sun, D.; Lin, X.; Deng, X.; He, N.; Zheng, S. J. Chem. Technol. Biotechnol. 2005, 80, 285.

(286) Darouiche, R. O. N. Engl. J. Med. 2004, 350, 1422.

(287) Mayer, K. H.; Schoenbaum, S. C. Prog. Cardiovasc. Dis. 1982, $25,43$.

(288) Costerton, J. W.; Stewart, P. S.; Greenberg, E. P. Science 1999, 284,1318 .

(289) Stewart, P. S.; Costerton, J. W. Lancet 2001, 358, 135.

(290) Hetrick, E. M.; Schoenfisch, M. H. Chem. Soc. Rev. 2006, 35, 780.

(291) Lear, G.; Lewis, G. D. Microbial Biofilms; Caister Academic Press: Norfolk, 2012.

(292) Bunyan, J.; Dilock, A. T.; Cawthorne, M. A.; Green, J. Br. J. Nutr. 1968, 22, 165.

(293) Mahmoudi, M.; Azadmanesh, K.; Shokrgozar, M. A.; Journeay, W. S.; Laurent, S. Chem. Rev. 2011, 111, 3407.

(294) Zhou, Y.; Chen, W.; Itoh, H.; Naka, K.; Ni, Q.; Yamane, H.; Chujo, Y. Chem. Commun. 2001, 23, 2518.

(295) Bauer, T. W.; Parvizi, J.; Kobayashi, N.; Krebs, V. J. Bone Jt. Surg. 2006, 88, 869.

(296) Ray, S.; Das, A. K.; Banerjee, A. Chem. Commun. 2006, 26, 2816.

(297) Pourjavadi, A.; Soleyman, R. J. Nanopart. Res. 2011, 13, 4647.

(298) Slocik, J. M.; Naik, R. R.; Stone, M. O.; Wright, D. W. J. Mater. Chem. 2005, 15, 749.

(299) Monroe, D. PLoS Biol. 2007, 5, e307.

(300) Vatamaniuk, O. K.; Mari, S.; Lu, Y.-P.; Rea, P. A. J. Biol. Chem. 2000, 275, 31451 .

(301) Maillard, M.; Huang, P.; Brus, L. Nano Lett. 2003, 3, 1611.

(302) Fan, Z.; Govorov, A. O. Nano Lett. 2010, 10, 2580.

(303) Bologna, R. A.; Tu, L. M.; Polansky, M.; Fraimow, H. D.; Gordon, D. A.; Whitmore, K. E. Urology 1999, 54, 982.

(304) Bosetti, M.; Masse, A.; Tobin, E.; Cannas, M. Biomaterials 2002, 23, 887 .

(305) Piedade, A. P.; Vieira, M. T.; Martins, A.; Silva, F. Nanotechnology 2007, 18, 105103.
(306) Masse, A.; Bruno, A.; Bosetti, M.; Biasibetti, A.; Cannas, M.; Gallinaro, P. J. Biomed. Mater. Res. 2000, 53, 600.

(307) Sheehan, E.; McKenna, J.; Mulhall, K. J.; Marks, P.; McCormack, D. J. Orthop. Res. 2004, 22, 39.

(308) Rai, M.; Yadav, A.; Gade, A. Biotechnol. Adv. 2009, 27, 76.

(309) Kumar, R.; Munstedt, H. Biomaterials 2005, 26, 2081.

(310) Lee, D.; Cohen, R. E.; Rubner, M. F. Langmuir 2005, 21, 9651.

(311) Sant, S. B.; Gill, K. S.; Burrell, R. E. Acta Biomater. 2007, 3, 341.

(312) Gosheger, G.; Hardes, J.; Ahrens, H.; Streitburger, A.; Buerger, H.; Erren, M.; Gunsel, A.; Kemper, F. H.; Winkelmann, W.; von Eiff, C. Biomaterials 2004, 25, 5547.

(313) Hardes, J.; Ahrens, H.; Gebert, C.; Streitbuerger, A.; Buerger, H.; Erren, M.; Gunsel, A.; Wedemeyer, C.; Saxler, G.; Winkelmann, W.; Gosheger, G. Biomaterials 2007, 28, 2869.

(314) Schierholz, J. M.; Lucas, L. J.; Rump, A.; Pulverer, G. J. Hosp. Infect. 1998, 40, 257.

(315) Diaz-Visurraga, J.; Garcia, A.; Cardenas, G. J. Appl. Microbiol. 2010, 108, 633.

(316) Martinez-Gutierrez, F.; Olive, P.; Banuelos, A.; Orrantia, E.; Nino, N.; Sanchez, E.; Ruiz, F.; Bach, H.; Av-Gay, Y. Nanomedicine 2010, 6, 681.

(317) Vasilev, K.; Sah, V. R.; Goreham, R. V.; Ndi, C.; Short, R. D.; Griesser, H. J. Nanotechnology 2010, 21, 215102.

(318) Bayston, R.; Vera, L.; Mills, A.; Ashraf, W.; Stevenson, O.; Howdle, S. M. J. Antimicrob. Chemother. 2010, 65, 258.

(319) Balan, L.; Schneider, R.; Lougnot, D. J. Prog. Org. Coat. 2008, 62,351 .

(320) Kong, H.; Jang, J. Langmuir 2008, 24, 2051.

(321) Shao, W.; Zhao, Q. Colloids Surf., B 2010, 76, 98.

(322) Shao, W.; Zhao, Q. Surf. Coat. Technol. 2010, 204, 1288.

(323) Burgers, R.; Eidt, A.; Frankenberger, R.; Rosentritt, M.; Schweikl, H.; Handel, G.; Hahnel, S. Arch. Oral. Biol. 2009, 54, 595.

(324) Valappil, S. P.; Pickup, D. M.; Carroll, D. L.; Hope, C. K.; Pratten, J.; Newport, R. J.; Smith, M. E.; Wilson, M.; Knowles, J. C. Antimicrob. Agents Chemother. 2007, 51, 4453.

(325) Zhou, W.; Zhong, X.; Wu, X.; Yuan, L.; Zhao, Z.; Wang, H.; Xia, Y.; Feng, Y.; He, J.; Chen, W. Surf. Coat. Technol. 2006, 200, 6155.

(326) Pan, J.; Liao, H.; Leygraf, C.; Thierry, D.; Li, J. J. Biomed. Mater. Res., Part B 1998, 40, 244.

(327) Watanabe, I.; McBride, M.; Newton, P.; Kurtz, K. S. Dent. Mater. 2009, 25, 629.

(328) Huang, H.-L.; Chang, Y.-Y.; Lai, M.-C.; Lin, C.-R.; Lai, C.-H.; Shieh, T.-M. Surf. Coat. Technol. 2010, 205, 1636.

(329) Liu, H.; Chen, Q.; Song, L.; Ye, R.; Lu, J.; Li, H. J. Non-Cryst. Solids 2008, 354, 1314

(330) Poelstra, K. A.; Barekzi, N. A.; Rediske, A. M.; Felts, A. G.; Slunt, J. B.; Grainger, D. W. J. Biomed. Mater. Res. 2002, 60, 206.

(331) Yao, C.; Perla, V.; McKenzie, J. L.; Slamovich, E. B.; Webster, T. J. J. Biomed. Nanotechnol. 2005, 1, 68.

(332) Li, J.; Wang, J.; Shen, L.; Xu, Z.; Li, P.; Wan, G.; Huang, N. Surf. Coat. Technol. 2007, 201, 8155.

(333) Singh, A. J. Appl. Polym. Sci. 2011, 119, 1084.

(334) Lin, L.; Gong, W.; Wang, X.; Li, X.; Wang, S. Mater. Lett. 2011, 65, 1375.

(335) Damm, C.; Münstedt, H.; Rösch, A. J. Mater. Sci.: Mater. Med. 2007, 42, 6067.

(336) Stobie, N.; Duffy, B.; Hinder, S. J.; McHale, P.; McCormack, D. E. Colloids Surf., B 2009, 72, 62.

(337) Stobie, N.; Duffy, B.; Colreavy, J.; McHale, P.; Hinder, S. J.; McCormack, D. E. J. Colloid Interface Sci. 2010, 345, 286.

(338) Zampino, D.; Ferreri, T.; Puglisi, C.; Mancuso, M.; Zaccone, R.; Scaffaro, R.; Bennardo, D. J. Mater. Sci.: Mater. Med. 2011, 46, 6734.

(339) Kassaee, M. Z.; Akhavan, A.; Sheikh, N.; Sodagar, A. J. Appl. Polym. Sci. 2008, 110, 1699.

(340) Santa Maria, L. C. de; Souza, J. D. C.; Aguiar, M. R. M. P.; Wang, S. H.; Mazzei, J. L.; Felzenszwalb, I.; Amico, S. C. J. Appl. Polym. Sci. 2008, 107, 1879. 
(341) Travan, A.; Pelillo, C.; Donati, I.; Marsich, E.; Benincasa, M.; Scarpa, T.; Semeraro, S.; Turco, G.; Gennaro, R.; Paoletti, S. Biomacromolecules 2009, 10, 1429.

(342) Travan, A.; Marsich, E.; Donati, I.; Benincasa, M.; Giazzon, M.; Felisari, L.; Paoletti, S. Acta Biomater. 2011, 7, 337.

(343) Kumar, R.; Münstedt, H. Polym. Int. 2005, 54, 1180.

(344) Kumar, R.; Howdle, S.; Munstedt, H. J. Biomed. Mater. Res., Part B 2005, 75, 311.

(345) Damm, C.; Münstedt, H.; Rösch, A. Mater. Chem. Phys. 2008, $108,61$.

(346) Boswald, M.; Mende, K.; Bernschneider, W.; Bonakdar, S.; Ruder, H.; Kissler, H.; Sieber, E.; Guggenbichler, J. P. Infection 1999, 27, S38-42.

(347) Karchmer, T. B.; Giannetta, E. T.; Muto, C. A.; Strain, B. A.; Farr, B. M. Arch. Intern. Med. 2000, 160, 3294.

(348) Plowman, R.; Graves, N.; Esquivel, J.; Roberts, J. A. J. Hosp. Infect. 2001, 48, 33.

(349) Ilić, V.; Šaponjić, Z.; Vodnik, V.; Lazović, S.; Dimitrijević, S.; Jovančić, P.; Nedeljković, J. M.; Radetić, M. Ind. Eng. Chem. Res. 2010, 49, 7287.

(350) Pollini, M.; Russo, M.; Licciulli, A.; Sannino, A.; Maffezzoli, A. J. Mater. Sci.: Mater. Med. 2009, 20, 2361.

(351) Cartmell, S. H.; Doherty, P. J.; Rhodes, N. P.; Hunt, J. A.; Healy, D. M.; Gilchrist, T. J. Mater. Sci.: Mater. Med. 1998, 9, 1.

(352) Alt, V.; Bechert, T.; Steinrucke, P.; Wagener, M.; Seidel, P.; Dingeldein, E.; Domann, E.; Schnettler, R. Biomaterials 2004, 25 4383.

(353) Chen, W.; Liu, Y.; Courtney, H.; Bettenga, M.; Agrawal, C.; Bumgardner, J.; Ong, J. Biomaterials 2006, 27, 5512.

(354) Xu, X.; Zhou, M. Fibers Polym. 2008, 9, 685.

(355) Liu, Y.; Liu, X.; Wang, X.; Yang, J.; Yang, X.-J.; Lu, L. J. Appl. Polym. Sci. 2010, 116, 2617.

(356) Schneider, O. D.; Loher, S.; Brunner, T. J.; Schmidlin, P.; Stark, W. J. J. Mater. Chem. 2008, 18, 2679.

(357) Malcher, M.; Volodkin, D.; Heurtault, B.; André, P.; Schaaf, P.; Möhwald, H.; Voegel, J.-C.; Sokolowski, A.; Ball, V.; Boulmedais, F.; Frisch, B. Langmuir 2008, 24, 10209.

(358) Qureshi, A. T.; Monroe, W. T.; Lopez, M. J.; Janes, M. E.; Dasa, V.; Park, S.; Amirsadeghi, A.; Hayes, D. J. J. Appl. Polym. Sci. 2011, 120, 3042.

(359) Masters, J. R. Nat. Rev. Cancer 2002, 2, 315.

(360) Balazs, D.; Triandafillu, K.; Wood, P.; Chevolot, Y.; van Delden, C.; Harms, H.; Hollenstein, C.; Mathieu, H. Biomaterials 2004, 25, 2139.

(361) Monteiro, D. R.; Gorup, L. F.; Takamiya, A. S.; Ruvollo-Filho, A. C.; de Camargo, E. R.; Barbosa, D. B. Int. J. Antimicrob. Agents 2009, 34, 103.

(362) Berra, L.; Kolobow, T.; Laquerriere, P.; Pitts, B.; Bramati, S.; Pohlmann, J.; Marelli, C.; Panzeri, M.; Brambillasca, P.; Villa, F.; Baccarelli, A.; Bouthors, S.; Stelfox, H.; Bigatello, L.; Moss, J.; Pesenti, A. Intensive Care Med. 2008, 34, 1030.

(363) Robin, A. Y.; Meuwly, M.; Fromm, K. M.; Goesmann, H.; Bernardinelli, G. CrystEngComm 2004, 6, 336.

(364) Fromm, K. M.; Gueneau, E. D.; Robin, A. Y.; Maudez, W.; Sague, J.; Bergougnant, R. Z. Anorg. Allg. Chem. 2005, 631, 1725.

(365) Sagué, J. L.; Fromm, K. M. Cryst. Growth Des. 2006, 6, 1566.

(366) Robin, A. Y.; Sague, J. L.; Fromm, K. M. CrystEngComm 2006, $8,403$.

(367) Robin, A. Y.; Sague Doimeadios, J. L.; Neels, A.; Vig Slenters, T.; Fromm, K. M. Inorg. Chim. Acta 2007, 360, 212.

(368) Slenters, T. V.; Sagué, J. L.; Brunetto, P. S.; Zuber, S.; Fleury, A.; Mirolo, L.; Robin, A. Y.; Meuwly, M.; Gordon, O.; Landmann, R.; Daniels, A. U.; Fromm, K. M. Materials 2010, 3, 3407.

(369) Gschwind, F.; Fromm, K. M. CrystEngComm 2012, 14, 4008.

(370) Brunetto, P. S.; Fromm, K. M. Chimia 2008, 62, 249.

(371) Brunetto, P. S.; Slenters, T. V.; Fromm, K. M. Materials 2011 4, 355 .

(372) Slenters, T. V.; Hauser-Gerspach, I.; Daniels, A. U.; Fromm, K. M. J. Mater. Chem. 2008, 18, 5359.
(373) Amalric, J.; Mutin, P. H.; Guerrero, G.; Ponche, A.; Sotto, A.; Lavigne, J.-P. J. Mater. Chem. 2008, 19, 141.

(374) Boks, N. P.; Norde, W.; van der Mei, H. C.; Busscher, H. J. Microbiology 2008, 154, 3122.

(375) Chou Chih-Wei, H.; Shan-hui, W. P.-H. J. Biomed. Mater. Res. 2008, 84A, 785 .

(376) Ward, R.; Anderson, J.; McVenes, R.; Stokes, K. J. Biomed. Mater. Res. 2007, 80A, 34.

(377) Lee, H.-Y.; Kampf, J. W.; Park, K. S.; Marsh, E. N. G. Cryst. Growth Des. 2008, 8, 296.

(378) Fu, J.; Ji, J.; Fan, D.; Shen, J. J. Biomed. Mater. Res., Part A 2006 79,665 .

(379) Serizawa, T.; Yamaguchi, M.; Akashi, M. Biomacromolecules 2002, 3, 724 .

(380) Huang, L.; Dai, T.; Xuan, Y.; Tegos, G. P.; Hamblin, M. R. Antimicrob. Agents Chemother. 2011, 55, 3432.

(381) Cao, X. L.; Cheng, C.; Ma, Y. L.; Zhao, C. S. J. Mater. Sci.: Mater. Med. 2010, 21, 2861.

(382) Kim, H. J.; Lee, H. C.; Oh, J. S.; Shin, B. A.; Oh, C. S.; Park, R. D.; Yang, K. S.; Cho, C. S. J. Biomater. Sci., Polym. Ed. 1999, 10, 543.

(383) Lu, S.; Gao, W.; Gu, H. Y. Burns 2008, 34, 623.

(384) Mi, F.-L.; Wu, Y.-B.; Shyu, S.-S.; Schoung, J.-Y.; Huang, Y.-B.; Tsai, Y.-H.; Hao, J.-Y. J. Biomed. Mater. Res. 2002, 59, 438.

(385) Ong, S.-Y.; Wu, J.; Moochhala, S.; Tan, M.-H.; Lu, J. Biomaterials 2008, 29, 4323.

(386) Sanpui, P.; Murugadoss, A.; Prasad, P.; Ghosh, S.; Chattopadhyay, A. Int. J. Food Microbiol. 2008, 124, 142.

(387) Cao, H.; Liu, X.; Meng, F.; Chu, P. Biomaterials 2011, 32, 693.

(388) Shmeleva, I. N.; Mikhailenko, N. Y.; Batrak, I. K. Glass Ceram. 1997, 54, 25.

(389) Granqvist, C. G. Sol. Energy Mater. Sol. Cells 2012, 99, 166.

(390) Kuroda, K.; Okido, M. Bioinorg. Chem. Appl. 2012, 730693.

(391) Surmenev, R. A. Surf. Coat. Technol. 2011, 206, 2035.

(392) Janssen, R.; Scheppokat, S.; Claussen, N. J. Eur. Ceram. Soc 2008, 28, 1369.

(393) Taylor, P.; Omotoso, O.; Wiskel, J.; Mitlin, D.; Burrell, R. Biomaterials 2005, 26, 7230.

(394) Taylor, P. L.; Ussher, A. L.; Burrell, R. E. Biomaterials 2005, 26, 7221.

(395) Ando, Y.; Miyamoto, H.; Noda, I.; Sakurai, N.; Akiyama, T.; Yonekura, Y.; Shimazaki, T.; Miyazaki, M.; Mawatari, M.; Hotokebuchi, T. Mater. Sci. Eng., C 2010, 30, 175.

(396) Kwok, S.; Zhang, W.; Wan, G.; McKenzie, D.; Bilek, M.; Chu, P. Diamond Relat. Mater. 2007, 16, 1353.

(397) Gray, J. E.; Norton, P. R.; Alnouno, R.; Marolda, C. L.; Valvano, M. A.; Griffiths, K. Biomaterials 2003, 24, 2759.

(398) Gray, J.; Norton, P.; Griffiths, K. Thin Solid Films 2005, 484, 196.

(399) Gray, J.; Norton, P.; Griffiths, K. Appl. Surf. Sci. 2003, 217, 210.

(400) Wen, T.; Gao, J.; Bian, B.; Shen, J. Mater. Lett. 2001, 50, 124

(401) Huang, C.; Becker, M. F.; Keto, J. W.; Kovar, D. J. Appl. Phys. 2007, 102, 54308 .

(402) Albert, A. D.; Becker, M. F.; Keto, J. W.; Kovar, D. Acta Mater. 2008, 56, 1820 .

(403) Dowling, D. P. Thin Solid Films 2001, 398-399, 602.

(404) Dong, Y.; Li, X.; Tian, L.; Bell, T.; Sammons, R. L.; Dong, H. Acta Biomater. 2011, 7, 447.

(405) Dellasega, D.; Facibeni, A.; Di Fonzo, F.; Bogana, M.; Polissi, A.; Conti, C.; Ducati, C.; Casari, C. S.; Li Bassi, A.; Bottani, C. E. Nanotechnology 2008, 19, 475602.

(406) Waterhouse, G. I. N.; Bowmaker, G. A.; Metson, J. B. Phys. Chem. Chem. Phys. 2001, 3, 3838.

(407) Antelman, M. S. U.S. Patent 5,211,855, 1993; http://www. rexresearch.com/antelman/silverox.htm\#5211855.

(408) Kawashita, M.; Tsuneyama, S.; Miyaji, F.; Kokubo, T.; Kozuka, H.; Yamamoto, K. Biomaterials 2000, 21, 393.

(409) Yuan, W.; Ji, J.; Fu, J.; Shen, J. J. Biomed. Mater. Res., Part B 2008, 85, 556. 
(410) Sabbani, S.; Gallego-Perez, D.; Nagy, A.; Waldman, W. J.; Hansford, D.; Dutta, P. K. Microporous Mesoporous Mater. 2010, 135, 131.

(411) Casemiro, L. A.; Martins, C. H. G.; Pires-de-Souza, F. d. C. P.; Panzeri, H. Gerodontology 2008, 25, 187.

(412) Oh, K.-T.; Joo, U.-H.; Park, G.-H.; Hwang, C.-J.; Kim, K.-N. J. Biomed. Mater. Res. 2006, 76, 306.

(413) Khan, A. N.; Usman, A. J. Alloys Compd. 2010, 491, 209.

(414) Song, D.-H.; Uhm, S.-H.; Lee, S.-B.; Han, J.-G.; Kim, K.-N. Thin Solid Films 2011, 519, 7079.

(415) Moseke, C.; Gbureck, U.; Elter, P.; Drechsler, P.; Zoll, A.; Thull, R.; Ewald, A. J. Mater. Sci.: Mater. Med. 2011, 22, 2711.

(416) Kang, D.-K.; Moon, S.-K.; Oh, K.-T.; Choi, G.-S.; Kim, K.-N. J. Biomed. Mater. Res., Part B 2009, 90B, 446.

(417) Wu, J.; Hou, S.; Ren, D.; Mather, P. T. Biomacromolecules 2009, 10, 2686.

(418) Radheshkumar, C.; Münstedt, H. Mater. Lett. 2005, 59, 1949.

(419) Gibbins, B.; Warner, L. Med. Device Diagn. Ind. 2005; http:// www.mddionline.com/article/role-antimicrobial-silvernanotechnology.

(420) dela Riviere, A. B.; Dossche, K. M.; Birnbaum, D. E.; Hacker, R. J. Heart Valve Dis. 2000, 9, 123.

(421) Olson, M. E.; Harmon, B. G.; Kollef, M. H. Chest 2002, 121, 863.

(422) Klasen, H. J. Burns 2000, 26, 117.

(423) Hollinger, M. A. Crit. Rev. Toxicol. 1996, 26, 255.

(424) Ersek, R. A.; Denton, D. R. Ann. Plast. Surg. 1984, 13, 482.

(425) Chu, C. S.; McManus, A. T.; Pruitt, B. A.; Masson, A. D. J. Trauma 1988, 28, 1488.

(426) Liedberg, H.; Lundeberg, T. Br. J. Urol. 1990, 65, 379.

(427) Johnson, J. R.; Roberts, P. L.; Olsen, R. J.; Moyer, K. A.; Stamm, W. E. J. Infect. Dis. 1990, 162, 1145.

(428) Stamm, W. E. Am. J. Med. 1991, 91, S65.

(429) Babycos, C.; Barrocas, A.; Webb, W. R. JPEN, J. Parenter. Enteral Nutr. 1993, 17, 61.

(430) Tweden, K. S.; Cameron, J. D.; Razzouk, A. J.; Holmberg, W. R.; Kelly, S. J. J. Heart Valve Dis. 1997, 6, 553.

(431) Muangman, P.; Chuntrasakul, C.; Silthram, S.; Suvanchote, S.; Benjathanung, R.; Kittidacha, S.; Rueksomtawin, S. J. Med. Assoc. Thailand 2006, 89, 953.

(432) Lansdown, A. B. G. Curr. Probl. Dermatol. 2006, 33, 17.

(433) http://www.teachpe.com/anatomy/respiratory_system.php.

(434) Casals, E.; Vazquez-Campos, S.; Bastus, N. G.; Puntes, V. Trends Anal. Chem. 2008, 27, 672.

(435) Kim, Y. S.; Song, M. Y.; Park, J. D.; Song, K. S.; Ryu, H. R.; Chung, Y. H.; Chang, H. K.; Lee, J. H.; Oh, K. H.; Kelman, B. J.; Hwang, I. K.; Yu, I. J. Part. Fibre Toxicol. 2010, 7, 1.

(436) http//www.lenntech.com/periodic/water/silver-and-water. htm.

(437) Loeschner, K.; Hadrup, N.; Qvortrup, K.; Larsen, A.; Gao, X.; Vogel, U.; Mortensen, A.; Lam, H.; Larsen, E. H. Part. Fibre Toxico.l 2011, 8, 1 .

(438) Trop, M.; Novak, M.; Rodl, S.; Hellbom, B.; Kroell, W.; Goessler, W. J. Trauma 2006, 60, 648.

(439) Wright, J. B.; Hansen, D. L.; Burrell, R. E. Wounds 1998, 10, 179.

(440) Warriner, R.; Burrell, R. Adv. Skin Wound Care 2005, 18, 2.

(441) Monafo, W. W.; Freedman, B. Burns 1987, 67, 133.

(442) Fox, C. L., Jr. Arch. Surg. 1968, 96, 184.

(443) Carsin, H.; Wassermann, D.; Pannier, M.; Dumas, R.; Bohbot, S. J. Wound Care 2004, 13, 145.

(444) Hidalgo, E.; Bartolomé, R.; Moreno, A.; Dominguez, C. Skin Pharmacol. Appl. 1998, 11, 140.

(445) Wright, J. B.; Lam, K.; Buret, A. G.; Olson, M.; Burrell, R. E. Wound Rep. Reg. 2002, 10, 141.

(446) Braydisch-Stolle, L.; Hussain, S.; Schlager, J. J.; Hofmann, M.C. Toxicol. Sci. 2005, 88, 412.

(447) Hussain, S. M.; Hess, K. L.; Gearhart, J. M.; Geiss, K. T.; Schlager, J. J. Toxicol. In Vitro 2005, 19, 975.
(448) Lee, A.-R. C.; Leem, H.; Lee, J.; Park, K. C. Biomaterials 2005, 26, 4670 .

(449) Fraser, J. F.; Cuttle, L.; Kempf, M.; Kimble, R. M. Aust. N. Z. J. Surg. 2004, 74, 139.

(450) Lam, P. K.; Chan, E. S. Y.; Ho, W. S.; Liew, C. T. Br. J. Biomed. Sci. 2004, 61, 125.

(451) Hansson, C.; Faergemann, J. Acta Derm.-Venereol. 1995, 75, 31.

(452) Sasaki, T.; Ohno, T. Toxicol. In Vitro 1994, 8, 1113.

(453) Kjolseth, D.; Frank, J. M.; Berker, J. H.; Anderson, G. L.; Rosenthal, A. I.; Acland, R. D.; Schuschke, D.; Campbell, F. R.; Tobin, G. R.; Weiner, L. J. J. Am. Coll. Surg. 1994, 179, 305.

(454) Poon, V. K. M.; Burd, A. Burns 2004, 30, 140.

(455) Hussain, S.; Anner, R. M.; Anner, B. M. Biochem. Biophys. Res. Commun. 1992, 189, 1444.

(456) Tsipouras, N.; Rix, C. J.; Brady, P. H. Clin. Chem. 1995, 41, 87.

(457) Dunn, K.; Edwards-Jones, V. Burns 2004, 30, S1.

(458) Innes, M.; Umraw, N.; Fish, J. S.; Gomez, M.; Cartotto, C. Burns 2001, 27, 621.

(459) Sheridan, R. L.; Petras, L.; Lydon, M.; Salvo, P. M. J. Burn Care Rehabil. 1997, 18, 139.

(460) Wan, A. T.; Conyers, R. A. J.; Coombs, C. J.; Masterton, J. P. Clin. Chem. 1991, 37, 1683.

(461) Nirmala, R.; Nam, K. T.; Park, D. K.; Woo-il, B.; Navamathavan, R.; Kim, H. Y. Surf. Coat. Technol. 2010, 205, 174.

(462) Oei, J. D.; Zhao, W. W.; Chu, L.; DeSilva, M. N.; Ghimire, A.; Rawls, H. R.; Whang, K. J. Biomed. Mater. Res. 2012, 100, 409.

(463) Ghafari Nazari, A.; Tahari, A.; Moztarzadeh, F.; Mozafari, M.; Bahrololoom, M. Micro Nano Lett. 2011, 6, 713

(464) Quirynen, M.; Gijbels, F.; Jacobs, R. Periodontology 2000, 33, 129.

(465) Hardes, J.; Streitburger, A.; Ahrens, H.; Nusselt, T.; Gebert, C.; Winkelmann, W.; Battmann, A.; Gosheger, G. Sarcoma 2007, 26539.

(466) Zheng, Z.; Yin, W.; Zara, J. N.; Li, W.; Kwak, J.; Mamidi, R.; Lee, M.; Siu, R. K.; Ngo, R.; Wang, J.; Carpenter, D.; Zhang, X.; Wu, B.; Ting, K.; Soo, C. Biomaterials 2010, 31, 9293.

(467) Branemark, P. I.; Hansson, B. O.; Adell, R.; Breine, U.; Lindström, J.; Hallén, O.; Ohman, A. Scand. J. Plast. Reconstr. Surg. Suppl. 1977, 16, 1.

(468) Ahmad, M.; McCarthy, M. B.; Gronowicz, G. Biomaterials $1999,20,211$

(469) Albrektsson, T.; Johansson, C. Eur. Spine J. 2001, 10, S96.

(470) Ku, C.-H.; Pioletti, D. P.; Browne, M.; Gregson, P. J. Biomaterials 2002, 23, 1447.

(471) Okumura, A.; Goto, M.; Goto, T.; Yoshinari, M.; Masuko, S.; Katsuki, T.; Tanaka, T. Biomaterials 2001, 22, 2263.

(472) Espehaug, B.; Furnes, O.; Havelin, L. I.; Engesaeter, L. B.; Vollset, S. E. J. Bone Jt. Surg. 2002, 84-B, 832.

(473) Cavalu, S.; Simon, V.; Goller, G.; Akin, I. Dig. J. Nanomater. Bios. 2011, 6, 779.

(474) Cavalu, S.; Simon, V.; Banica, F. Dig. J. Nanomater. Bios. 2010, $6,89$.

(475) Webster, T. J.; Ejiofor, J. U. Biomaterials 2004, 25, 4731.

(476) Nath, S.; Kalmodia, S.; Basu, B. J. Mater. Sci.: Mater. Med. 2010, 21, 1273.

(477) Khandwekar, A. P.; Patil, D. P.; Shouche, Y. S.; Doble, M. J. Med. Biol. Eng. 2009, 29, 84.

(478) Hsu, S.-H.; Tseng, H.-J.; Lin, Y.-C. Biomaterials 2010, 31, 6796. (479) Prabhakar, P. K.; Raj, S.; Anuradha, P. R.; Sawant, S. N.; Doble, M. Colloids Surf., B 2011, 86, 146.

(480) Multanen, M.; Talja, M.; Tammela, T. L. J.; Seppälä, J.; Välimaa, T.; Järvi, K.; Törmälä, P. Urol. Res. 2001, 29, 113.

(481) Badiou, W.; Lavigne, J.-P.; Bousquet, P.-J.; O’Callaghan, D.; Marès, P.; de Tayrac, R. Int. Urogynecol. J. 2011, 22, 265.

(482) www.smart-drugs.com/JamesSouth-silver.htm.

(483) Agency for Toxic Substances and Disease Registry Public Health Statement. Toxicological Profile for Silver; U.S. Department of Health and Human Services, Public Health Service: Atlanta, GA, 1990; http://www.atsdr.cdc.gov/toxprofiles/tp146.pdf. 
(484) Schrand, A. M.; Rahman, M.; Hussain, S. M.; Schlager, J. J.; Smith, D. A.; Syed, A. F. Nanomed. Nanobiotechnol. 2010, 2, 544.

(485) van Wijnhoven, S. W.; Peijnenburg, W. J. G. M.; Herberts, C. A.; Hagens, W. I.; Oomen, A. G.; Heugens, E. H. W.; Roszek, B.; Bisschops, J.; Gosens, I.; van de Meent, D.; Dekkers, S.; de Jong, W. H.; van Zijverden, M.; Sips, A. J. A. M.; Geertsma, R. E. Nanotoxicology 2009, 3, 109.

(486) Rattanaruengsrikul, V.; Pimpha, N.; Supaphol, P. J. Appl. Polym. Sci. 2012, 124, 1668.

(487) Geronemus, R. G.; Mertz, P. M.; Eaglstein, W. H. Arch. Dermatol. 1979, 115, 1311.

(488) http://cira.ornl.gov/.

(489) Material Safety Data Sheet Silver nitrate CAS\# 7761-88-8.

(490) Material Safety Data Sheet Silver Oxide CAS\# 20667-12-3.

(491) Hultman, P.; Eneström, S.; Turley, S. J.; Pollard, K. M. Clin. Exp. Immunol. 1994, 96, 285.

(492) Armitage, S. A.; White, M. A.; Wilson, H. K. Ann. Occup. Hyg. 1996, 40, 331

(493) U.S. Environmental Protection Agency: Integrated Risk Information System Silver (CASRN 7440-22-4); http://www.epa. gov/iris/subst/0099.htm.

(494) Drake, P.; Hazelwood, K. J. Ann. Occup. Hyg. 2005, 49, 575.

(495) Yamamoto, A.; Honna, R.; Sumita, M. J. Biomed. Mater. Res.,

Part A 1998, 39, 331.

(496) Rosenman, K. D.; Moss, A.; Kon, S. J. Occup. Med. 1979, 21, 430.

(497) Sue, Y.-M.; Lee, J. Y.-Y.; Wang, M.-C.; Lin, T.-K.; Sung, J.-M.; Huang, J.-J. Am. J. Kidney Dis. 2001, 37, 1048.

(498) Christensen, F. M.; Johnston, H. J.; Stone, V.; Aitken, R. J.; Hankin, S.; Peters, S.; Aschberger, K. Nanotoxicology 2010, 4, 284.

(499) Takenaka, S.; Karg, E.; Roth, C.; Schulz, H.; Ziesenis, A.; Heinzmann, U.; Schramel, P.; Heyder, J. Environ. Health Perspect. 2001, 109, 547.

(500) Wadhera, A.; Fung, M. Dermatol. Online J. 2005, 11, 12.

(501) Ji, J. H.; Jung, J. H.; Kim, S. S.; Yoon, J.-U.; Park, J. D.; Choi, B. S.; Chung, Y. H.; Kwon, I.; Jeong, J.; Han, B.; Shin, J. H.; Sung, J. H.; Song, K. S.; Yu, I. J. Inhalation Toxicol. 2007, 19, 857.

(502) Kim, Y. S.; Kim, J. S.; Cho, H. S.; Rha, D. S.; Kim, J. M.; Park, J. D.; Choi, B.; Lim, R.; Chang, H. K.; Chung, Y. H.; Kwon, I. H.; Jeong, J.; Han, B. S.; Yu, I. L. Inhalation Toxicol. 2008, 20, 575.

(503) Sung, J. H.; Ji, J. H.; Park, J. D.; Yoon, J. U.; Kim, D. S.; Jeon, K. S.; Song, M. Y.; Jeong, J.; Han, B. S.; Han, J. H.; Chung, Y. H.; Chang, H. K.; Lee, J. H.; Cho, M. H.; Kelman, B. J.; Yu, I. J. Toxicol. Sci. 2009, 108, 452 .

(504) Maitre, S.; Jaber, K.; Perrot, J.-L.; Guy, C.; Cambazard, F. Ann. Dermatol. Venereol. 2002, 129, 217.

(505) Di Baldi, C.; Minoia, C.; Nucci, C.; Capodaglio, E.; Manzo, L. Toxicol. Lett. 1988, 41, 261.

(506) Rosenblatt, M.; Cymet, T. J. Am. Osteopath. Assoc. 1987, 87, 509.

(507) Ohbo, Y.; Fukuzako, H.; Takeuchi, K.; Takigawa, M. Psychiatry Clin. Neurosci. 1996, 50, 89.

(508) Mirsattari, S. M.; Hammond, R. R.; Sharpe, M. D.; Leung, F. Y.; Young, G. B. Neurology 2004, 62, 1408.

(509) Allee, W. C.; Schuett, J. F. Biol. Bull. 1927, 53, 303.

(510) Shousse, S. S.; Whipple, G. H. J. Exp. Med. 1931, 53, 413.

(511) Horner, H. C.; Roebuck, B. D.; Smith, R. P. Drug Chem. Toxicol. 1983, 6, 267.

(512) Sudmann, E.; Vik, H.; Rait, M.; Todnem, K.; Andersen, K.-J.; Julsham, K.; Flesland, O.; Rungby, J. Med. Prog. Technol. 1994, 20, 179.

(513) Vik, H.; Andersen, K. J.; Julshamn, J.; Todnem, K. Lancet 1985, $325,872$.

(514) Ellender, G.; Ham, K. N. Br. J. Exp. Pathol. 1989, 70, 21.

(515) Butany, J.; Leask, R. L.; Desai, N. D.; Jegatheeswaran, A.; Silversides, C. J. Cardiovasc. Surg. 2006, 21, 530.

(516) Schaff, H.; Carrel, T. P.; Jamieson, W. R. E.; Jones, K. W.; Rufilanchas, J. J.; Cooley, D. A.; Hetzer, R.; Stumpe, F.; Grunkemeier, G. L.; Kennard, E. D.; Holibkov, R. Ann. Thorac. Surg. 2002, 73, 785.

(517) Kim, S.; Choi, I.-H. Yonsei Med. J. 2012, 53, 654.
(518) AshaRani, P. V.; Hande, M. P.; Valiyaveettil, S. BMC Cell Biol. 2009, 10, 65.

(519) Petros, R. A.; DeSimone, J. M. Nat. Rev. Drug Discover 2010, 9, 615.

(520) Pérez-Martínez, F. C.; Guerra, J.; Posadas, I.; Ceña, V. Pharm. Res. 2011, 28, 1843.

(521) Greulich, C.; Diendorf, J.; Simon, T.; Eggeler, G.; Epple, M.; Köller, M. Acta Biomater. 2011, 7, 347.

(522) Iversen, T.-G.; Skotland, T.; Sandvig, K. Nano Today 2011, 6, 176

(523) Kawata, K.; Osawa, M.; Okabe, S. Environ. Sci. Technol. 2009, 43, 6046.

(524) Kim, J.; Kim, S.; Lee, S. Nanotoxicology 2011, 5, 208.

(525) Kim, S.; Choi, J. E.; Choi, J.; Chung, K.-H.; Park, K.; Yi, J.; Ryu, D.-Y. Toxicol. In Vitro 2009, 23, 1076.

(526) Liu, W.; Wu, Y.; Wang, C.; Li, H. C.; Wang, T.; Liao, C. Y.; Cui, L.; Zhou, Q. F.; Yan, B.; Jiang, G. B. Nanotoxicology 2010, 4, 319.

(527) Park, E.-J.; Yi, J.; Kim, Y.; Choi, K.; Park, K. Toxicol. In Vitro 2010, 24, 872.

(528) Asharani, P.; Lianwu, Y.; Gong, Z.; Valiyaveettil, S. Nanotoxicology 2011, 5, 43.

(529) Kim, T.-H.; Kim, M.; Park, H.-S.; Shin, U. S.; Gong, M.-S.; Kim, H.-W. J. Biomed. Mater. Res., Part A 2012, 100A, 1033.

(530) Loghman, A.; Iraj, S. H.; Naghi, D. A.; Pejman, M. Afr. J. Biotechnol. 2012, 11, 6207.

(531) Arora, S.; Jain, J.; Rajwade, J. M.; Paknikar, K. M. Toxicol. Lett. 2008, 179, 90.

(532) Xia, T.; Kovochich, M.; Brant, J.; Hotze, M.; Sempf, J.; Oberley, T.; Sioutas, C.; Yeh, J. I.; Wiesner, M.; Nel, A. E. Nano Lett. 2006, 6, 1794.

(533) Slater, A. F.; Stefan, C.; Nobel, I.; van den Dobbelsteen, D. J.; Orrenius, S. Toxicol. Lett. 1995, 82, 149.

(534) Lubick, N. Environ. Sci. Technol. 2008, 42, 8617.

(535) Johnston, H. J.; Hutchison, G.; Christensen, F. M.; Peters, S.; Hankin, S.; Stone, V. Crit. Rev. Toxicol. 2010, 40, 328.

(536) Borm, P. J. A.; Kreyling, W. J. Nanosci. Nanotechnol. 2004, 4, 521.

(537) Takenaka, S.; Karg, E.; Roth, C.; Schulz, H.; Ziesenis, A.; Heinzmann, U.; Schramel, P.; Heyder, J. Environ. Health Perspect. 2001, 109, 547

(538) Asare, N.; Instanes, C.; Sandberg, W. J.; Refsnes, M.; Schwarze, P.; Kruszewski, M.; Brunborg, G. Toxicology 2012, 291, 65.

(539) Hamer, D. H. Annu. Rev. Biochem. 1986, 55, 913.

(540) Lansdown, A. B. G.; Sampson, B.; Laupattarakasen, P.; Vuttivirojana, A. Br. J. Dermatol. 1997, 137, 728.

(541) Ricketts, C. R.; Lowbury, E. J. L.; Lawrence, J. C.; Hall, M.; Wilkins, M. D. Br. Med. J. 1970, 2, 444.

(542) Spacciapoli, P.; Buxton, D.; Rothstein, D.; Friden, P. J. Periodontal Res. 2001, 36, 108.

(543) Burd, A.; Kwok, C. H.; Hung, S. C.; Chan, H. S.; Gu, H.; Lam, W. K.; Huang, L. Wound Rep. Reg. 2007, 15, 94.

(544) Peram, V.; Iftikhar, S.; Lietz, H.; Mobarhan, S.; Frommel, T. O. Cancer Lett. 1996, 106, 133.

(545) Bartold, P. M.; Raben, A. J. Periodontal Res. 1996, 31, 205.

(546) Aoyagi, H.; Katagiri, M. Dent. Mater. J. 2004, 23, 340.

(547) White, J. M. L.; Powell, A.; Brady, K.; Russell-Jones, R. Clin. Exp. Dermatol. 2003, 28, 254.

(548) Aoyagi, H.; Iwasaki, S.-I. Dent. Mater. J. 2008, 27, 612.

(549) Zelazowski, A. J.; Gasyna, Z.; Stillman, M. J. J. Biol. Chem. 1989, 264, 17091.

(550) Simon, G. J. Environ. Health Perspect. 1994, 102, 107.

(551) Solioz, M.; Odermatt, A.; Krapf, R. FEBS Lett. 1994, 346, 44.

(552) Silver, S. Mol. Microbiol. 1993, 10, 7.

(553) Tsai, K.-J.; Yoon, K. P.; Lynn, A. R. J. Bacteriol. 1992, 174, 116.

(554) Jaitvovich, A. A.; Bertorello, A. M. Semin. Nephrol. 2006, 26, 386.

(555) Petris, M.; Mercer, J. F. B.; Culvenor, J. G.; Lockhart, P.; Gleeson, P. A.; Camakaris, J. EMBO J. 1996, 15, 6084. 
(556) Verheijen, F. W.; Beerens, C. E.; Havelaar, A. C.; Kleijer, W. J.; Mancini, G. M. J. Med. Genet. 1998, 35, 849.

(557) Linz, R.; Lutsenko, S. J. Bioenerg. Biomembr. 2007, 39, 403.

(558) Barnes, N.; Tsivkovskii, R.; Tsivkovskaia, N.; Lutsenko, S. J. Biol. Chem. 2005, 280, 9640.

(559) Nyasae, L.; Bustos, R.; Braiterman, L.; Eipper, B.; Hubbard, A. Am. J. Physiol.: Gastrointest. Liver Physiol. 2007, 292, G1181.

(560) La Fontaine, S.; Mercer, J. F. B. Arch. Biochem. Biophys. 2007, 463, 149.

(561) Fanni, D.; Pilloni, L.; Orru, S.; Coni, P.; Liguori, C.; Serra, S.; Lai, M. L.; Uccheddu, A.; Contu, L.; Eyken, P.; Faa, G. Eur. J. Histochem. 2005, 49, 371.

(562) Mahli, H.; Irani, A. N.; Volenberg, I.; Scilsky, M. L.; Gupta, S. Gastroenterology 2002, 122, 438.

(563) Lee, J.; Marjorette, M.; Pena, O.; Nose, Y.; Thiele, D. J. J. Biol. Chem. 2002, 277, 4380.

(564) Petris, M. J.; Smith, K.; Lee, J.; Thiele, D. J. J. Biol. Chem. 2003, $278,9639$.

(565) Kim, H.; Son, H.-Y.; Bailey, S. M.; Lee, J. Am. J. Physiol.: Gastrointest. Liver Physiol. 2009, 296, G356.

(566) Kaler, S. G. Nat. Rev. Neurol. 2011, 7, 15.

(567) Furr, J. R.; Russell, A.; Turner, T. D.; Andrews, A. J. Hosp. Infect. 1994, 27, 201.

(568) Lansdown, A. B. G.; Jensen, K.; Jensen, M. Q. J. Wound Care 2003, 12, 205

(569) Thomas, S.; McCubbin, P. A. J. Wound Care 2003, 12, 101.

(570) Thomas, S.; McCubbin, P. A. J. Wound Care 2003, 12, 305.

(571) http://www.worldwidewounds.com/2004/february/Cooper/ Topical-Antimicrobial-Agents.html.

(572) Sun, T.; Jackson, S.; Haycock, J. W.; MacNeil, S. J. Biotechnol. 2006, 122, 372.

(573) Lloyd, J. R.; Byrne, J. M.; Coker, V. S. Curr. Opin. Biotechnol. 2011, 22, 509 . 